Aus der Klinik für Kardiologie und Pneumologie

(Prof. Dr. med. G. Hasenfuß)

der Medizinischen Fakultät der Universität Göttingen

\title{
Risikostratifizierung bei Patienten mit akuter Lungenembolie anhand der in der \\ Computertomographie abgeschätzten \\ Thrombuslast und des Verhältnisses von rechts- zu linksventrikulärem Diameter
}

\author{
INAUGURAL-DISSERTATION \\ zur Erlangung des Doktorgrades \\ der Medizinischen Fakultät der \\ Georg-August-Universität zu Göttingen
}

vorgelegt von

Judith Beatrice Reuter

aus

Düsseldorf

Göttingen 2019 
Dekan:

\section{Betreuungsausschuss}

Betreuerin:

Ko-Betreuer:

\section{Prüfungskommission}

Referent/in

Ko-Referent/in:

Drittreferent/in:

Datum der mündlichen Prüfung: 25.08.2020
Prof. Dr. med. C. Dellas

PD Dr. med. J. M. Sommerlath Sohns
Prof. Dr. med. C. Dellas

PD Dr. med. J. M. Sommerlath Sohns

Prof. Dr. med. M. Oppermann 
Hiermit erkläre ich, die Dissertation mit dem Titel "Risikostratifizierung bei Patienten mit akuter Lungenembolie anhand der in der Computertomographie abgeschätzten Thrombuslast und des Verhältnisses von rechts- zu linksventrikulärem Diameter" eigenständig angefertigt und keine anderen als die von mir angegebenen Quellen und Hilfsmittel verwendet zu haben.

Göttingen, den 


\section{Inhaltsverzeichnis}

Abbildungsverzeichnis .......................................................................... III

Tabellenverzeichnis .............................................................................................

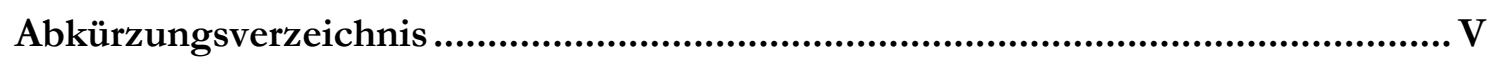

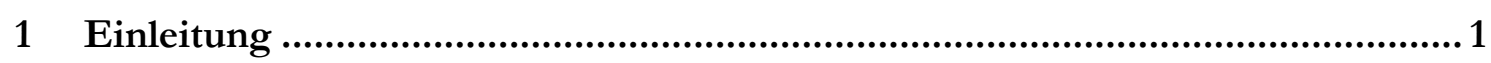

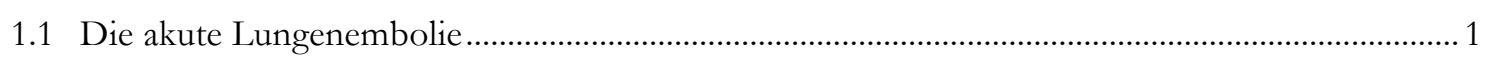

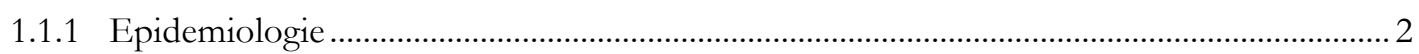

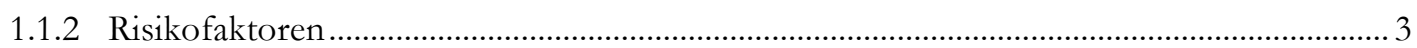

1.1.3 Symptomatik und Pathophysiologie des Rechtsherzversagens ............................................. 3

1.2 Risikostratifizierung der akuten Lungenembolie........................................................................ 5

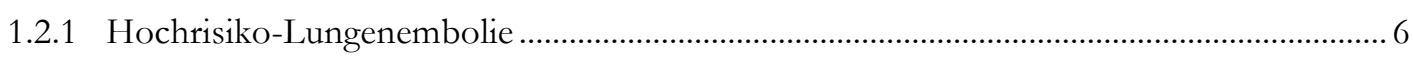

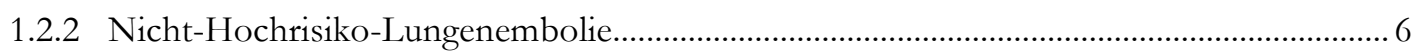

1.3 Therapie der akuten Lungenembolie ........................................................................................ 9

1.4 Die Computertomographie als Teil des diagnostischen Standardalgorithmus..............................10

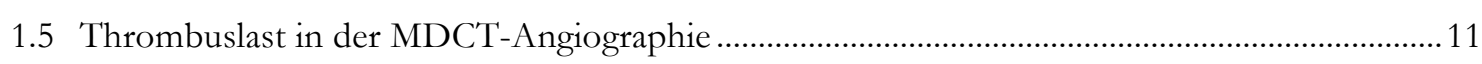

1.5.1 Vorhersagekraft von Thromben in zentralen Gefäßen.........................................................11

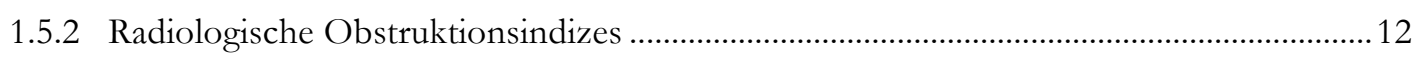

1.5.3 Vorhersagekraft der Thrombusgröße..............................................................................13

1.6 Bestimmung der rechtsventrikulären Dysfunktion.........................................................................

1.6.1 Aussagekraft der RV/LV-Ratio in der Computertomographie ...........................................14

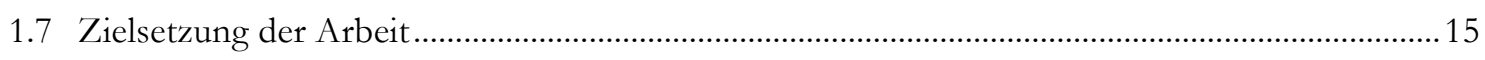

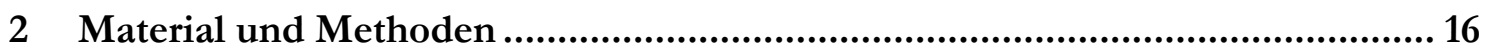

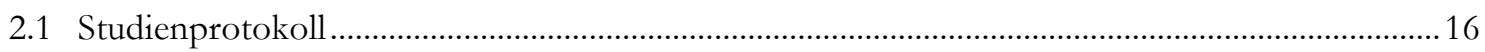

2.1.1 Ein- und Ausschlusskriterien............................................................................................. 16

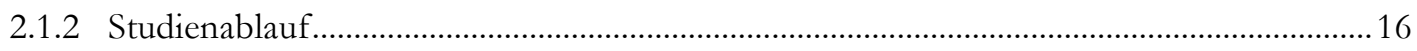

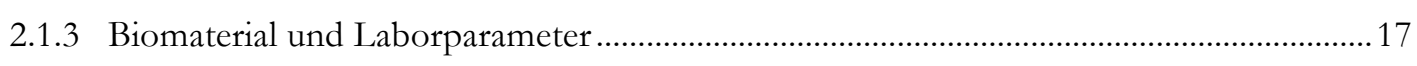

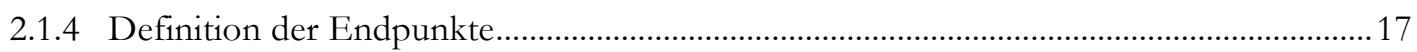

2.2 Technische Aspekte der Computertomographie ..............................................................................18

2.3 Durchführung der computertomographischen Auswertung .........................................................19

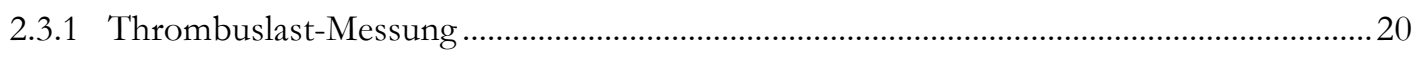

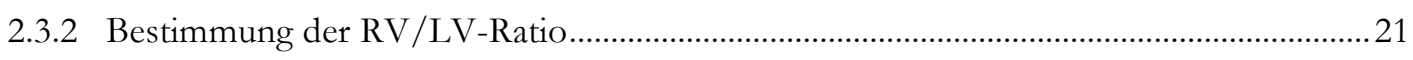

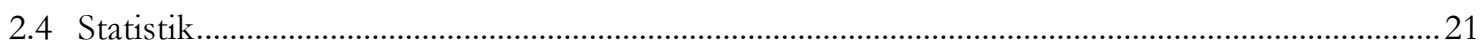




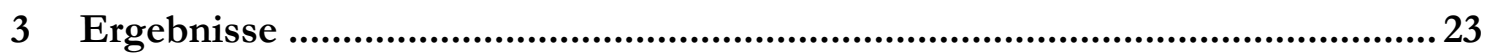

3.1 Beschreibung von Studienpopulation und primärem Endpunkt ................................................2

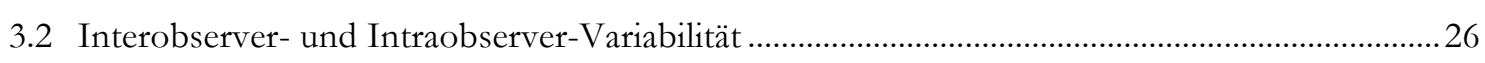

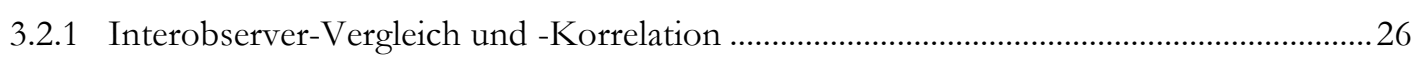

3.2.2 Interobserver-Variabilität Messwerterhebung 1 vs. 2 .......................................................... 30

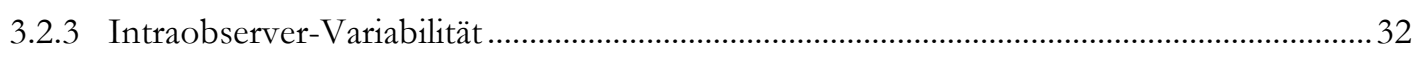

3.3 Erhobene computertomographische Befunde in der Studienpopulation ...................................... 33

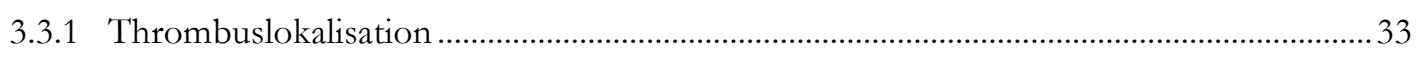

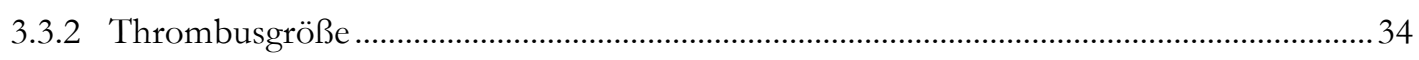

3.3.3 Rechtsventrikulärer Diameter und RV/LV-Ratio.................................................................. 34

3.4 Risikostratifizierung mittels Multidetektor-Computertomographie................................................. 35

3.4.1 Prädiktiver Wert der computertomographischen Parameter................................................. 35

3.4.2 Weiterführende Untersuchung der signifikanten Parameter ................................................ 36

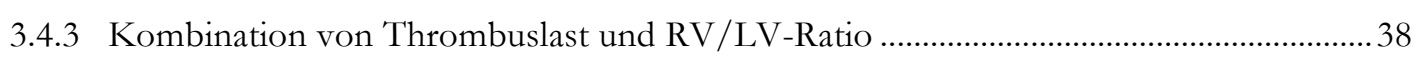

3.4.4 Subgruppenanalyse: Normotensive Patienten .......................................................................... 41

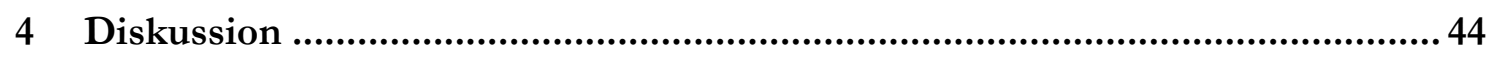

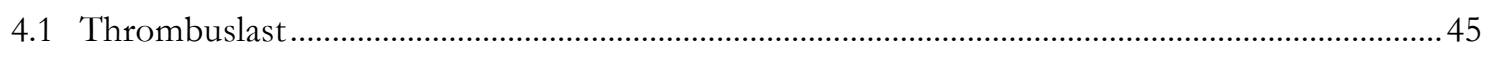

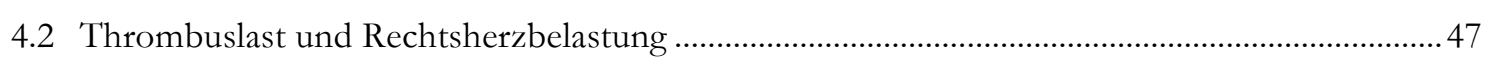

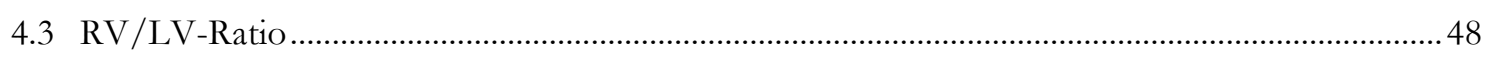

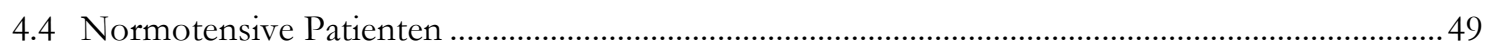

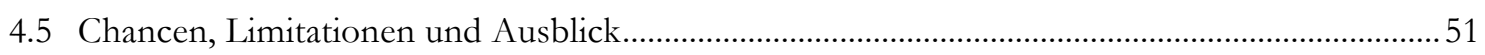

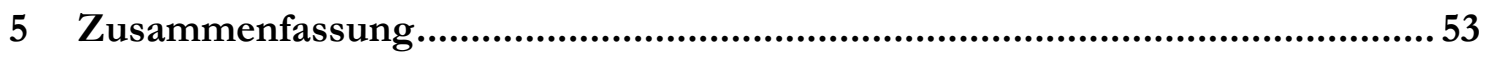

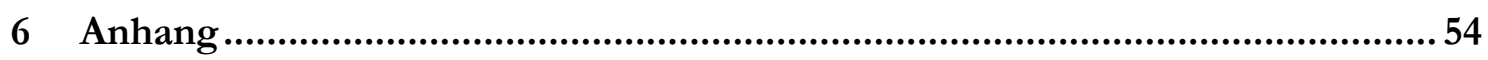

6.1 Patienteninformation und Einverständniserklärung zur Studienteilnahme ....................................54

6.2 Datenerfassungsbogen für das Pulmonary Embolism Registry Göttingen ....................................57

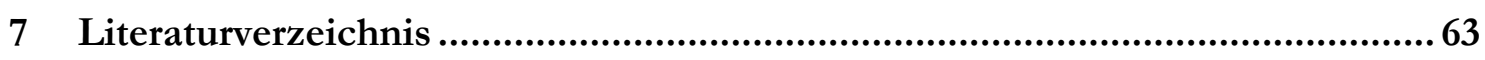




\section{Abbildungsverzeichnis}

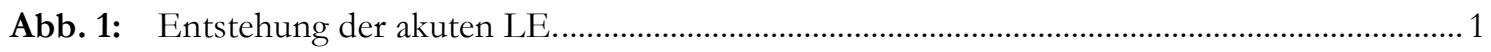

Abb. 2: Pathophysiologie des hämodynamischen Kollapses bei der akuten LE............................... 5

Abb. 3: Bestimmung des frühen Mortalitätsrisikos als Grundlage für das weitere Management von Patienten mit bestätigter akuter LE................................................................ 8

Abb. 4: Risikoadaptierte therapeutische Strategien der akuten LE. ................................................. 10

Abb. 5: Gründe für den Ausschluss von Patienten aus der retrospektiven CT-Auswertung..........19

Abb. 6 Vermessung des Thrombus- und Gefäßquerschnitts zur Bestimmung des prozentualen Gefäßverschlusses und reitender Thrombus im HS. ....................................................20

Abb. 7 Bestimmung des RV- und LV-Diameters und RV-Dilatation (RV>LV).........................21

Abb. 8: Korrelation der prozentualen Thrombuslast-Messungen U1 vs. U2, U1 vs. U3 und

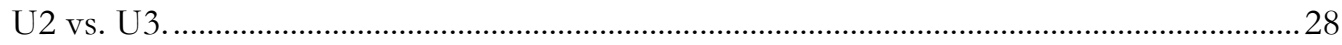

Abb. 9: Korrelation der RV/LV-Ratio U1 vs. U2, U1 vs. U3 und U2 vs. U3. .............................29

Abb. 10: Bland-Altman-Diagramm der Thrombuslast-Messungen von M1 und M2......................31

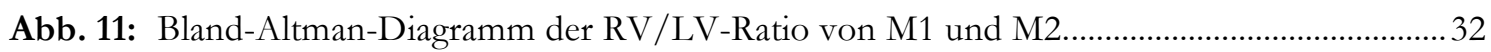

Abb. 12: ROC-Kurve der RV/LV-Ratio im Hinblick auf das Erreichen des primären EP...............37

Abb. 13: ROC-Kurve der RV/LV-Ratio bei Vorliegen eines zentralen Thrombus...........................39

Abb. 14: ROC-Kurve der RV/LV-Ratio bei Vorliegen einer Thrombuslast in LPA >50\% + $\mathrm{RPA}>50 \%$

Abb. 15: ROC-Kurve der RV/LV-Ratio bei Vorliegen einer Thrombuslast in LPA >50\% + $\mathrm{RPA}>50 \%$ im Hinblick auf das Erreichen des primären EP. 


\section{Tabellenverzeichnis}

Tab. 1: Berechnung des PESI-Scores und des sPESI-Scores zur Abschätzung des Mortalitätsrisikos bei Patienten mit Nicht-Hochrisiko-LE. ........................................................... 8

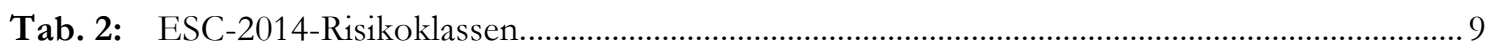

Tab. 3: Gegenüberstellung des Qanadli-Scores und des Mastora-Scores........................................13

Tab. 4: Charakteristika, Begleiterkrankungen, Risikofaktoren, klinische Präsentationen, ausgewählte Laborparameter und Risikoklassifikationen der Studienpopulation bei Aufnahme und bei Erreichen des primären EP.......................................................................2

Tab. 5: Häufigkeiten der EP und der Komplikationen innerhalb der ersten 30 Tage....................25

Tab. 6: Gesamte und gemeinsame CT-Auswertungen der Untersucher. .......................................26

Tab. 7: Abweichungen der Untersuchungsergebnisse aller drei Auswerter. ....................................227

Tab. 8: Koeffizienten der Thrombuslast und RV/LV-Ratio aller drei Untersucher. .....................227

Tab. 9: Bland-Altman-Analyse der Thrombuslast-Messungen von M1 und M2.........................3 31

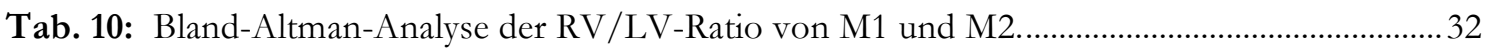

Tab. 11: Abweichungen und Korrelation der Untersuchungsergebnisse von U1 zu zwei ver-

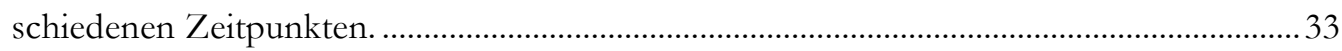

Tab. 12: Häufigkeiten der verschiedenen Thrombuslokalisationen................................................... 33

Tab. 13: Prozentuale Obstruktion der Pulmonalgefäße und Häufigkeiten ausgewählter Pro-

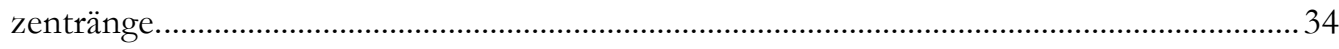

Tab. 14: Medianer RV-Diameter und RV/LV-Ratio mit beispielhaften Cut-off-Werten................. 35

Tab. 15: Prädiktiver Wert der CT-Parameter für das Erreichen des primären EP. ..........................36

Tab. 16: Prognostische Performances der Thrombuslokalisation im HS + LPA + RPA und der RV/LV-Ratio >1,3 vs. ausgewählte klinische und laborchemische Parameter............ 38

Tab. 17: Prädiktiver Wert der CT-Parameter für das Erreichen des primären EP. 42

Tab. 18: Prädiktiver Wert ausgewählter CT-Parameter für das Erreichen des primären EP bei Patienten mit nach ESC-2014-Score intermediär-hohem Risiko.....

Tab. 19: Prädiktiver Wert ausgewählter CT-Parameter für das Erreichen des primären EP bei Patienten mit nach ESC-2014-Score intermediär-niedrigem/niedrigem Risiko. 


\section{Abkürzungsverzeichnis}

AUC

BMI

BNP

chron.

COPD

CT

Echo

EKG

EP

ESC

GFR

H-FABP

HS

hs'TnT

$\mathrm{HZV}$

i.v.

ICC

IQR

KHK

KI

LE

LPA

LV

M1

M2

MDCT

MDRD

NPW

NT-proBNP

OR

PACS

$\mathrm{paO}_{2}$
Area Under the Curve

Body-Mass-Index

Brain Natriuretic Peptide

chronisch

chronisch-obstruktive Lungenerkrankung

Computertomographie

Echokardiographie

Elektrokardiographie

Endpunkt

Europäische Gesellschaft für Kardiologie

glomeruläre Filtrationsrate

Heart-type Fatty Acid Binding Protein

Pulmonalarterienhauptstamm

hoch sensitives Troponin $\mathrm{T}$

Herzzeitvolumen

intravenös

Intraclass-Korrelationskoeffizient

Interquartilsabstand

koronare Herzerkrankung

Konfidenzintervall

Lungenembolie

linke Pulmonalarterie

linker Ventrikel

Messwerterhebung 1

Messwerterhebung 2

Multidetektor-Computertomographie

Modification of Diet in Renal Disease

negativer prädiktiver Wert

N-Terminal Pro Brain Natriuretic Peptide

Odds Ratio

Picture Archiving and Communication System

arterieller Sauerstoffpartialdruck 
PAP pulmonalarterieller Druck

PERGO Pulmonary Embolism Registry Göttingen

PESI Pulmonary Embolism Severity Index

PPW positiver prädiktiver Wert

PROTECT Prognostic Value of CT Study

ROC Receiver-Operating-Characteristics-Analyse

RPA rechte Pulmonalarterie

RV rechter Ventrikel

RV/LV-Ratio Right-to-Left Ventricular Ratio

RVEDD rechtsventrikulärer enddiastolischer Diameter

s.c.

subkutan

$\mathrm{SaO}_{2} \quad$ pulsoxymetrisch gemessene Sauerstoffsättigung

sPESI Simplified Pulmonary Embolism Severity Index

TTE transthorakale Echokardiographie

TVT tiefe (Bein-)Venenthrombose

U1 Untersucher 1

U2 Untersucher 2

U3 Untersucher 3

VTE venöse Thromboembolie 


\section{$1 \quad$ Einleitung}

\subsection{Die akute Lungenembolie}

Die akute Lungenembolie (LE) stellt zusammen mit der tiefen Beinvenenthrombose (TVT) unter dem Überbegriff der venösen Thromboembolie (VTE) die dritthäufigste Erkrankung des Herz-Kreislauf-Systems dar (Konstantinides et al. 2014). Häufig präsentiert sie sich als medizinischer Notfall (Torbicki et al. 2008) und ist nach Myokardinfarkt und Schlaganfall die dritthäufigste kardiovaskuläre Todesursache (Goldhaber und Bounameaux 2012; Lankeit 2016).

Bei einer Lungenembolie kommt es zum Gefäßverschluss einer oder mehrerer Pulmonalarterien mit daraus resultierender distaler Minderperfusion. Als Embolus können Thromben, in selteneren Fällen Lipide, Luft oder Fremdmaterial nach Einschwemmung

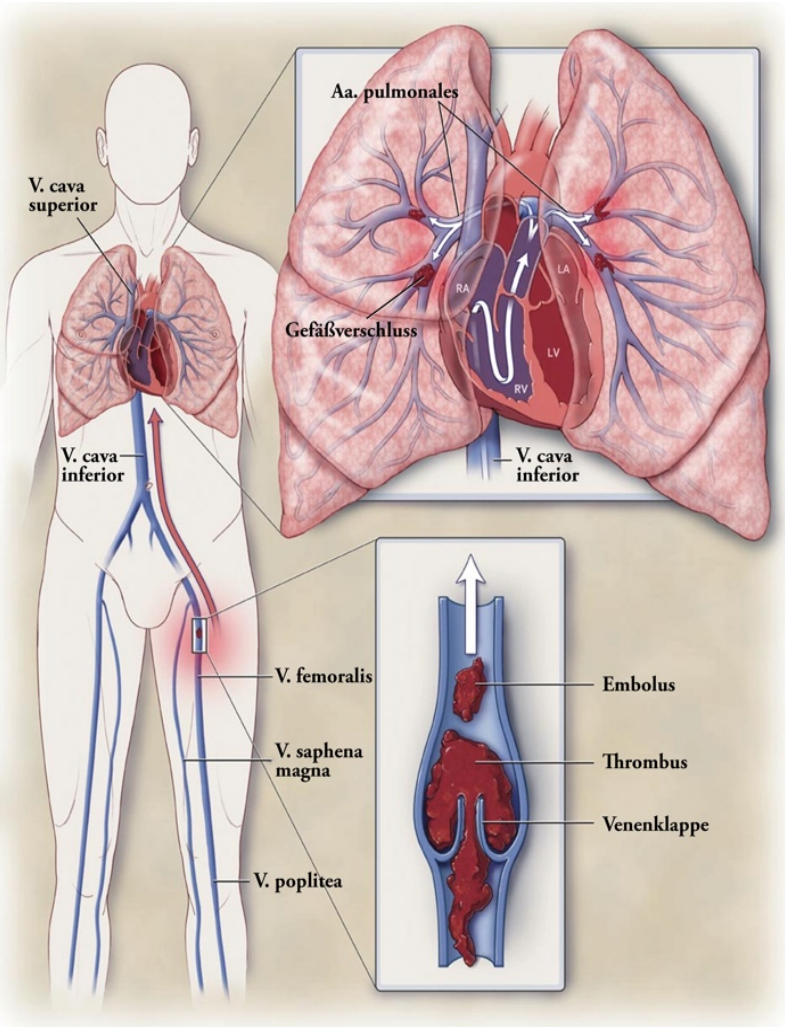

Abb. 1: Entstehung der akuten LE.

(Die Verwendung erfolgt mit freundlicher Genehmigung von Tapson 2008, Copyright Massachusetts Medical Society.) über den venösen Blutkreislauf fungieren (Goldhaber 2015; Lankeit 2016). Der ausschließliche Fokus dieser Arbeit liegt auf Thromben venösen Ursprungs. In über 90\% der Fälle (Lankeit 2016) entstehen diese in den tiefen Beinvenen und gelangen über die Vena cava inferior, den rechten Vorhof und Ventrikel in die Pulmonalarterien (Abb. 1). Seltener kann es auch zur Thrombusbildung in den oberen Extremitäten kommen. Ursachen hierfür sind zentrale Venenkatheter, Schrittmacher und implantierbare Kardioverter-Defibrillatoren (Goldhaber 2015; Lankeit 2016). 


\subsubsection{Epidemiologie}

Da die akute LE oft die Folge einer TVT darstellt, wird die Anzahl der neuaufgetretenen Erkrankungen beider Ereignisse in der Literatur unter der Inzidenz der VTE zusammengefasst angegeben (Konstantinides et al. 2014; Lankeit 2016). Untersuchungen in den USA und Europa legen ihre jährliche Inzidenz auf 100-200 Fälle pro 100.000 Einwohner fest (Cohen et al. 2007; Heit 2008; Goldhaber 2015; Heit et al. 2016) mit Unterschieden in Bevölkerungsgruppen verschiedener ethnischer Abstammung (Heit 2008; Heit et al. 2016). Das Risiko zur Erkrankung an einer VTE steigt mit zunehmendem Alter an und verdoppelt sich ab dem 40. Lebensjahr mit jedem weiterführenden Lebensjahrzehnt (Anderson und Spencer 2003; Konstantinides et al. 2014). In der Gruppe der unter 15-Jährigen finden sich weniger als fünf Fälle pro 100.000, in der Altersgruppe der 80-Jährigen wächst die Fallzahl exponentiell auf 500/100.000 (White 2003). Die VTE manifestiert sich klinisch bei einem Drittel der Patienten als akute LE und bei zwei Dritteln der Patienten als symptomatische TVT (White 2003). In einer retrospektiven bevölkerungsbasierten Kohortenstudie in Minnesota fand sich bei fast einem Viertel der Patienten als erste klinische Präsentation einer LE der plötzliche Tod (Heit et al. 1999). Das Fehlen routinemäßiger postmortaler Begutachtungen führt dazu, dass viele tödliche LEs unentdeckt bleiben, weshalb ihre Inzidenz unterschätzt wird (Cohen et al. 2007). In ihrer Studie fanden Cohen et al. 2007 heraus, dass 12\% aller jährlichen Todesfälle in Europa Folge einer LE sind. Die Erkrankung stellt damit einen bedeutsamen Grund für die Mortalität, Morbidität und Hospitalisation in europäischen Ländern dar (Konstantinides et al. 2014; Lankeit 2016). Je nach Studie liegt die Mortalität der LE bei 12\% innerhalb der ersten 30 Tage (White 2003), 17,4\% innerhalb der ersten drei Monate (Goldhaber et al. 1999) oder bei 21/100.000 Einwohner im Zeitraum eines Jahres (Olié et al. 2015). Heit et al. (1999) ermittelten in Überlebenszeitanalysen eine Überlebenswahrscheinlichkeit nach Auftreten einer LE von 66,8\% in 30 Tagen, 57,4\% in einem Jahr und 41,5\% in acht Jahren. Das Rezidivrisiko einer VTE wird von verschiedenen Autoren ebenfalls unterschiedlich eingeschätzt. In einer Studie beträgt das Wiederholungsrisiko innerhalb der ersten 30 Tage 5,2\%, innerhalb eines Jahres 12,9\% und innerhalb von zehn Jahren bis zu 30,4\%. Damit liegt es in den ersten sechs bis zwölf Monaten nach dem Erstereignis am höchsten (Heit et al. 2000; Heit 2016). Andere legen das Risiko für ein erneutes Ereignis zwischen 0,4\% und 10,7\% im Zeitraum eines Jahres (Prins et al. 2018) und auf $7,7 \%$ in zwei Jahren fest (Cushman et al. 2004). Eine weitere Studie fand ein höheres Rezidivrisiko: $17,5 \%$ in zwei Jahren, $24,6 \%$ in fünf Jahren und 30,3\% in acht Jahren (Prandoni et al. 1996). 


\subsubsection{Risikofaktoren}

Die Risikofaktoren für VTE können in permanente, patientenbezogene und temporäre, situationsbezogene Risikofaktoren unterteilt werden (Konstantinides et al. 2014). Eine VTE gilt als „provoziert“ in Gegenwart von zeitlich begrenzten oder reversiblen Risikofaktoren wie Operation, Trauma, Immobilisation, Schwangerschaft, Einnahme oraler Kontrazeptiva oder Hormonersatztherapie in den vorangegangenen sechs Wochen bis drei Monaten und als „unprovoziert“ in Abwesenheit derselben (Konstantinides et al. 2014). Eine akute LE ohne das Vorhandensein eines bekannten Risikofaktors tritt bei 30\% der Patienten auf (Konstantinides et al. 2014). Es findet eine Gewichtung der Risikofaktoren anhand ihrer Bedeutung für die Entwicklung einer VTE statt. Diese wird mittels des Chancenverhältnisses, der Odds Ratio (OR) angegeben, das zeigt, um welchen Faktor die Erkrankungswahrscheinlichkeit in der Population mit vorhandenem Risikofaktor erhöht ist gegenüber der Population ohne Risikofaktor. Zu den stark prädisponierenden Risikofaktoren $(\mathrm{OR}>10)$ zählen unter anderem große Operationen mit einer Anästhesiedauer >30 Minuten (Anderson und Spencer 2003), schwere Traumata, Frakturen der unteren Extremität, Hüft- oder Kniegelenksersatz und vorangegangene VTE (Konstantinides et al. 2014). Die Wahrscheinlichkeit für ein zweites VTE-Ereignis ist in der sich an das Erstereignis anschließenden Hoch-Risiko-Periode 8-fach erhöht (Anderson und Spencer 2003). Beispiele für moderate Risikofaktoren (OR 2-9) sind Thrombophilie, Chemo- oder Hormonersatztherapie und orale Kontrazeptiva (Konstantinides et al. 2014). Frauen, die regelmäßig Verhütungsmittel einnehmen, haben ein 3- bis 4-fach erhöhtes Risiko (Anderson und Spencer 2003). Maligne Grunderkrankungen zählen ebenfalls zu dieser Gruppe der moderaten Risikofaktoren, doch variiert ihr Risiko je nach Art der Erkrankung. Allen gemeinsam ist das höchste Risiko bei Vorliegen einer Metastasierung (Chew et al. 2006). Das Vorhandensein einer Krebserkrankung steigert die Gesamtmortalität sowie das Sterblichkeitsrisiko nach einer akuten LE (Gussoni et al. 2013; Konstantinides et al. 2014). Alter, arterielle Hypertonie, Diabetes mellitus und eine Immobilisation $>3$ Tage stellen schwache Prädispositionen für eine VTE dar (Konstantinides et al. 2014).

\subsubsection{Symptomatik und Pathophysiologie des Rechtsherzversagens}

Die akute LE kann aufgrund unspezifischer klinischer Präsentation und Symptomatik der unverzüglichen Diagnostik entgehen (Konstantinides et al. 2014; Goldhaber 2015). Ein komplett asymptomatisches klinisches Erscheinungsbild, bei dem die LE nur per Zufall im Rahmen anderer Diagnostik oder einer Autopsie entdeckt wird, bis hin zum kardiogenen 
Schock mit Reanimationspflichtigkeit ist möglich (Konstantinides et al. 2014). Stark hinweisgebend sind eine plötzlich neu einsetzende Dyspnoe sowie pleuritischer Thoraxschmerz und Husten. Bei Patienten mit kardialen oder pulmonalen Vorerkrankungen kann als einziges Symptom eine Verschlechterung der bereits bestehenden Luftnot vorkommen. Seltener präsentiert sich die akute LE mit retrosternalem Thoraxschmerz, Präsynkopen oder Synkopen. Hämoptysen, Fieber über $38^{\circ} \mathrm{C}$, progrediente Dyspnoe, Zyanose und die Zeichen einer TVT gehören zur weiteren unspezifischen Symptomatik (Miniati et al. 1999; Pollack et al. 2011; Konstantinides et al. 2014). In der Blutgasanalyse gehen Hypoxie und Hypokapnie häufig mit der akuten LE einher (Miniati et al. 1999; Konstantinides et al. 2014). Außerdem können eine Sinustachykardie, atriale Arrhythmien, Vorhofflimmern und Tachypnoe mit einer LE assoziiert sein (Miniati et al. 1999; Torbicki et al. 2008; Konstantinides et al. 2014). Als seltenes, aber überaus kritisches klinisches Bild sind arterielle Hypotension und Schock möglich (Konstantinides et al. 2014). Rechtsventrikuläres (RV-) Versagen als Folge einer erhöhten RV-Nachlast gilt als Haupttodesursache der akuten LE (Konstantinides et al. 2014) innerhalb der ersten 30 Tage nach Diagnosestellung (Kucher et al. 2005) (Abb. 2). Gefäßokklusion und eine LE-induzierte, mediatorvermittelte Vasokonstriktion der pulmonalarteriellen Gefäße sorgen dafür, dass der Pulmonalgefäßwiderstand erhöht und die arterielle Compliance erniedrigt wird. Als Resultat kommt es zu einer Dilatation des RV (Lankhaar et al. 2006; Konstantinides et al. 2014; Goldhaber 2015). Bei einer Obstruktion $>30-50 \%$ des arteriellen Gefäßbettes der Lunge steigt der mittlere pulmonalarterielle Druck (PAP) kompensatorisch an (McIntyre und Sasahara 1971; Konstantinides et al. 2014). Eine solche kurzzeitige Adaption zur Aufrechterhaltung der Lungendurchblutung und des systemischen Blutdrucks ist möglich, allerdings kann der dünnwandige, nicht prä-konditionierte RV keinen mittleren PAP $>40 \mathrm{mmHg}$ über eine längere Zeit generieren (Konstantinides et al. 2014; Agnelli und Becattini 2015). Die RV-Dilatation führt über eine Erhöhung der Wandspannung des Ventrikels und eine neurohumerale Aktivierung zur Myokardentzündung. Eine sekundäre hämodynamische Destabilisierung innerhalb der ersten 24-48 Stunden nach der akuten LE lässt sich möglicherweise durch diese Entzündungsreaktion erklären (Konstantinides et al. 2014). Die entstandene mangelhafte Sauerstoffversorgung des RV zieht als Konsequenz eine RV-Ischämie nach sich, die von pathophysiologischer Signifikanz in der Akutphase der LE ist (Konstantinides et al. 2014; Goldhaber 2015). Verminderter RV-Auswurf führt zur Senkung der linksventrikulären (LV-) Vorlast, welche wiederum ein vermindertes Herzzeitvolumen (HZV) und einen niedrigeren systemischen Blutdruck zur Folge hat. Die hieraus resultierende Minderperfusion der Koronararterien trägt über eine mangelhafte Sauerstoffversorgung des Herzens als weiterer Faktor 
zum RV- und globalen kardialen Pumpversagen bei. Es folgt der kardiogene Schock und schlussendlich der Exitus letalis (Konstantinides et al. 2014; Agnelli und Becattini 2015; Goldhaber 2015).

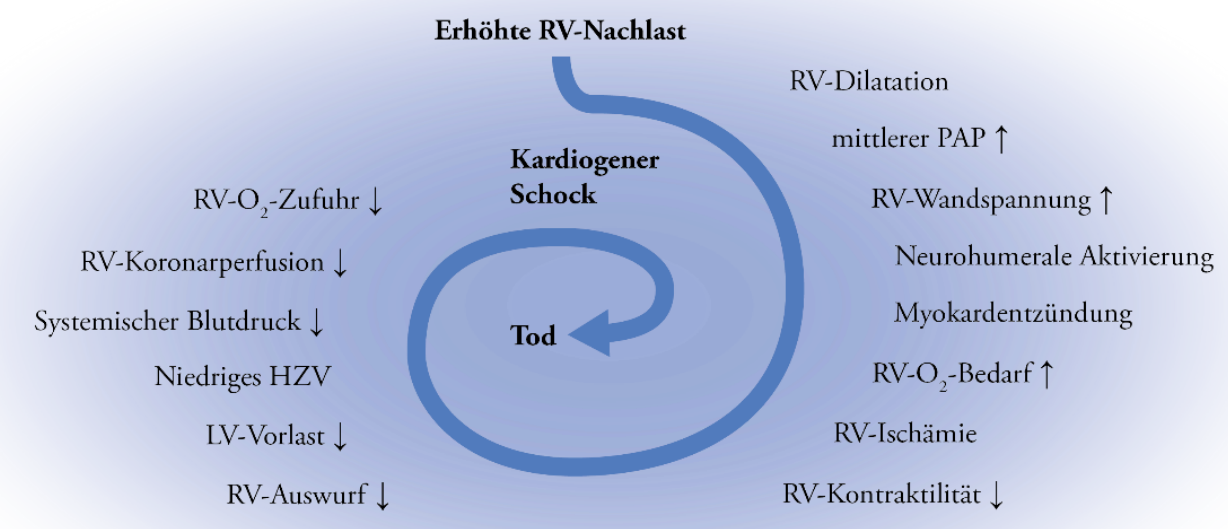

Abb. 2: Pathophysiologie des hämodynamischen Kollapses bei der akuten LE (modifiziert nach Konstantinides et al. 2014).

\subsection{Risikostratifizierung der akuten Lungenembolie}

Besteht bei einem Patienten aufgrund seines klinischen Erscheinungsbildes der Verdacht auf eine akute LE gibt es zur weiteren Diagnostik und zum therapeutischen Management von der Europäischen Gesellschaft für Kardiologie (ESC) verfasste Leitlinien (Konstantinides et al. 2014). Dabei findet aufgrund der klinischen Präsentation eine initiale Einteilung in Hochrisiko-LE-Patienten und Nicht-Hochrisiko-LE-Patienten statt. Diese Einteilung des Schweregrades der akuten LE berücksichtigt das deutlich höhere Mortalitätsrisiko innerhalb der ersten 30 Tage der Patienten, die bereits initial hypotensiv sind oder sich im Schock befinden und bestimmt den weiteren Algorithmus zur Diagnosesicherung (Konstantinides et al. 2014). 


\subsubsection{Hochrisiko-Lungenembolie}

Hochrisiko-LE-Patienten präsentieren sich hämodynamisch instabil. Dies wird definiert als kardiogener Schock oder systolischer Blutdruck $<90 \mathrm{mmHg}$ oder als Abfall des systolischen Blutdrucks von $>40 \mathrm{mmHg}$ für mehr als 15 Minuten (Konstantinides et al. 2014). Als diagnostische Erstmaßnahme wird eine computertomographische (CT-) Angiographie mit Kontrastmittel zur Bestätigung oder zum Ausschluss einer akuten LE durchgeführt (Konstantinides et al. 2014). Sollte eine CT-Angiographie aufgrund des kritischen Zustandes des Patienten oder aus logistischen Gründen nicht möglich sein, so wird eine transthorakale Echokardiographie (TTE) am Patientenbett zum Nachweis einer RV-Dysfunktion durchgeführt (Konstantinides et al. 2014). Es müssen außerdem wichtige Differentialdiagnosen wie ein akutes Koronarsyndrom, eine Aortendissektion, eine Herzbeuteltamponade oder eine akute Herzklappendysfunktion ausgeschlossen werden (Konstantinides et al. 2014). Bei einem instabilen Patienten reicht ein für das Vorliegen einer LE positiver Befund der TTE als Grundlage für reperfundierende Maßnahmen aus. Es sind keine weiteren Tests notwendig (Konstantinides et al. 2014).

\subsubsection{Nicht-Hochrisiko-Lungenembolie}

Besteht bei einem Patienten keine hämodynamische Instabilität wird er initial der Gruppe mit Nicht-Hochrisiko-LE zugeordnet. Zur weiteren Bestimmung der klinischen Wahrscheinlichkeit einer LE finden mehrere Scores Anwendung wie zum Beispiel der WellsScore und der revidierte Genfer Score (Konstantinides et al. 2014). Beide Scores basieren auf einfach zugänglichen Patienteninformationen. Bei Zuordnung zur Gruppe derer mit hoher Wahrscheinlichkeit für das Vorliegen einer akuten LE sind die betreffenden Patienten in ca. 60\% der Fälle tatsächlich erkrankt. Ergeben die Scores eine mittlere Wahrscheinlichkeit so liegt noch bei ca. 29\% der Patienten eine akute LE vor und bei einer niedrigen Wahrscheinlichkeitsvorhersage besteht eine Prävalenz von ca. 10\% (Ceriani et al. 2010; Konstantinides et al. 2014). Die vereinfachten Versionen beider Scores nutzen ein System mit zwei Kategorien. Hier wird nur zwischen dem wahrscheinlichen und dem unwahrscheinlichen Vorliegen einer akuten LE unterschieden (Wells et al. 2000; Konstantinides et al. 2014). Ergibt die Auswertung dieser Scores eine hohe Wahrscheinlichkeit für das Vorliegen einer LE, so findet im Anschluss eine CT-Angiographie mit Kontrastmittel zum Ausschluss oder zur Bestätigung der Verdachtsdiagnose statt (Konstantinides et al. 2014). Liefern die Ergebnisse der Scores hingegen nur eine niedrige bis mittlere Wahrscheinlichkeit für das Vorliegen einer LE, wird zunächst eine D-Dimer-Bestimmung durchgeführt. 
Ungefähr 30\% der Verdachtsfälle können hierdurch bereits ausgeschlossen werden (Konstantinides et al. 2014). D-Dimere sind im Plasma bei gleichzeitigem Vorliegen von Koagulation und Fibrinolyse erhöht. Aufgrund ihres hohen negativen prädiktiven Wertes (NPW) $(97,3 \%)$ (Wells et al. 2001) machen normale D-Dimer-Level eine akute LE oder eine TVT bei niedriger klinischer Wahrscheinlichkeit unwahrscheinlich. Erhöhte Werte hingegen dienen nicht zur alleinigen Bestätigung der Verdachtsdiagnose, da für ihren Anstieg diverse Ursachen in Frage kommen wie beispielsweise eine maligne Grunderkrankung, eine Entzündung oder eine Nekrose (Konstantinides et al. 2014). Werden erhöhte D-Dimere gemessen, erfolgt im Anschluss ein CT mit Kontrastmittel zur endgültigen Diagnosesicherung (Konstantinides et al. 2014). Nach Bestätigung der akuten LE wird das frühe Mortalitätsrisiko unter Verwendung klinischer Risikoscores wie des Pulmonary Embolism Severity Index (PESI) (Aujesky et al. 2005) oder des simplifizierten PESI (sPESI) (Tab. 1) ermittelt (Jiménez et al. 2010a; Konstantinides et al. 2014) (Abb. 3). Anhand dieser Scores kann zwischen einem niedrigen (PESI-Klasse I-II oder sPESI =0) und einem erhöhten (PESIKlasse III-V oder sPESI $\geq 1$ ) Mortalitätsrisiko unterschieden werden. Besteht bei einem hämodynamisch stabilen Patienten (=Nicht-Hochrisiko-LE) ein sPESI von $0 \mathrm{bzw}$. eine PESI-Klasse von I-II, so wird er der Niedrigrisikogruppe zugeordnet. Ansonsten besteht ein intermediäres Risiko. Bei Annahme eines intermediären Risikos wird durch bildgebende Verfahren (z.B. TTE, CT) und/oder anhand von Biomarkern beurteilt, ob eine RVDysfunktion oder eine myokardiale Schädigung vorliegen. Bestimmt werden hierfür die Konzentrationen von Brain Natriuretic Peptide (BNP), N-Terminal Pro Brain Natriuretic Peptide (NT-proBNP), Troponinen oder Heart-type Fatty Acid Binding Protein (H-FABP) (Konstantinides et al. 2014). Sind sowohl Bildgebung als auch Biomarker positiv, so besteht bei den Patienten ein intermediär-hohes Risiko für Komplikationen, andernfalls ein intermediärniedriges Risiko (Tab. 2). 
Tab. 1: Berechnung des PESI-Scores und des sPESI-Scores (modifiziert nach Aujesky et al. 2005; Jiménez et al. 2010a) zur Abschätzung des Mortalitätsrisikos bei Patienten mit Nicht-Hochrisiko-LE.

\begin{tabular}{|l|c|c|}
\hline \multicolumn{1}{|c|}{ Parameter } & PESI-Score & sPESI-Score \\
\hline Alter & Alter in Jahren & $>80$ Jahre: +1 \\
männliches Geschlecht & +10 & +1 \\
Krebserkrankung & +30 & +13 \\
Herzinsuffizienz & +10 & +1 \\
chronische Lungenerkrankung & +10 & +1 \\
Herzfrequenz $\geq 110$ min $^{-1}$ & +20 & \\
systolischer Blutdruck $<100 \mathrm{mmHg}$ & +30 & \\
Atemfrequenz $\geq 30$ min $^{-1}$ & +20 & +1 \\
Temperatur $<36^{\circ} \mathrm{C}$ & +20 & \\
neuaufgetretene psychische Auffälligkeit ${ }^{1}$ & +60 & \\
arterielle Sauerstoffsättigung $<90 \%{ }^{2}$ & +20 & $0=1,0 \%$ \\
\hline & 665 Klasse I & \\
Punkte & $86-105$ Klasse II & 30 -d-Mortalitätsrisiko: \\
& $106-125$ Klasse IV & $\geq 1=10,9 \%$ \\
\hline
\end{tabular}

${ }^{1}$ definiert als: Desorientiertheit, Lethargie, Stupor, Koma; ${ }^{2}$ mit oder ohne Sauerstoffgabe; ${ }^{3}$ Herzinsuffizienz und chronische Lungenerkrankung werden zusammengefasst als chronische kardiopulmonale Erkrankung.

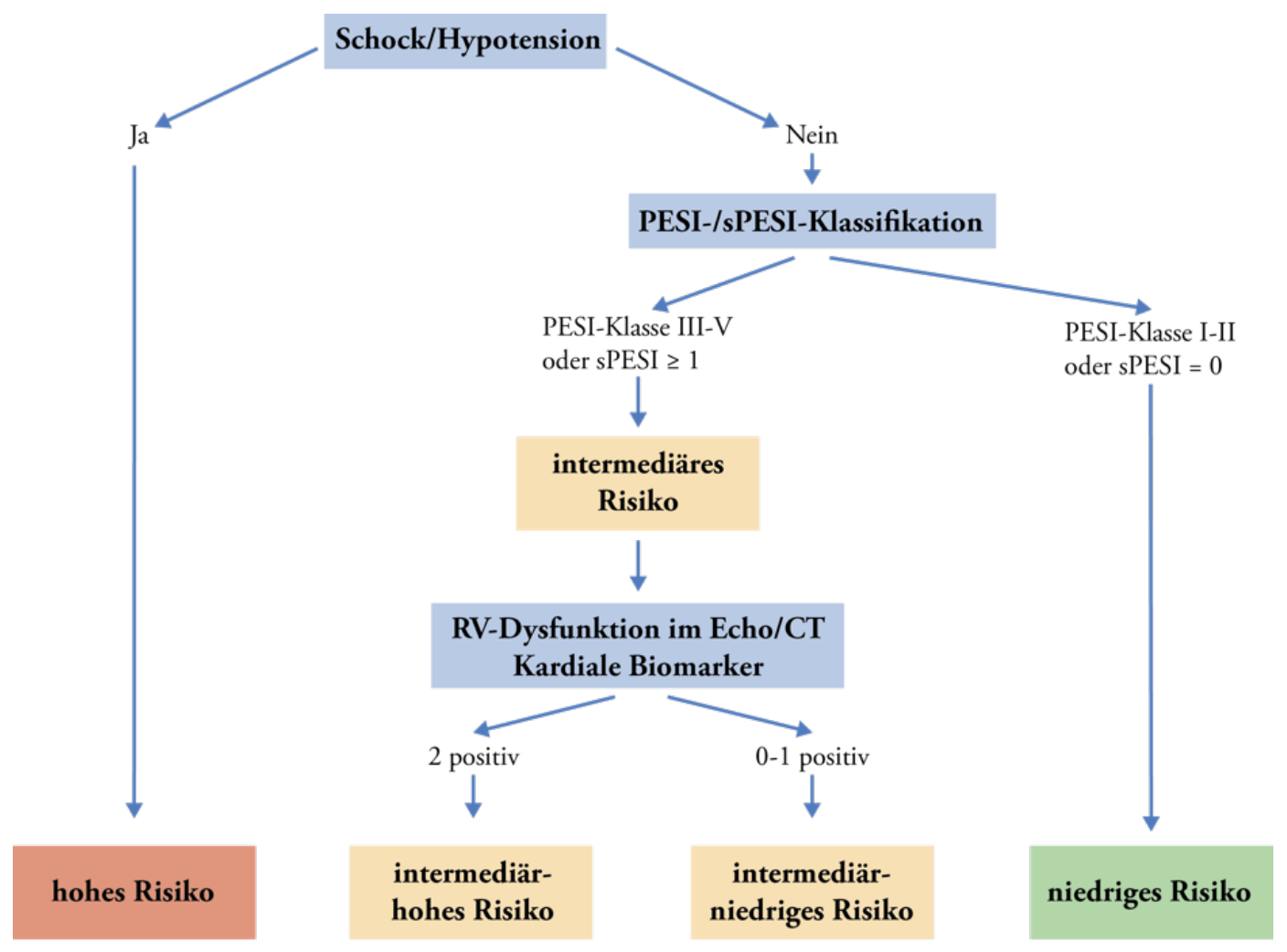

Abb. 3: Bestimmung des frühen Mortalitätsrisikos als Grundlage für das weitere Management von Patienten mit bestätigter akuter LE (modifiziert nach Konstantinides et al. 2014). 
Tab. 2: ESC-2014-Risikoklassen (modifiziert nach Konstantinides et al. 2014).

\begin{tabular}{|l|c|c|c|c|}
\hline \multicolumn{1}{|c|}{$\begin{array}{c}\text { frühes } \\
\text { Mortalitätsrisiko }\end{array}$} & $\begin{array}{c}\text { Schock/ } \\
\text { Hypotension }\end{array}$ & $\begin{array}{c}\text { PESI-Klasse III-V } \\
\text { oder sPESI } \geq \mathbf{1}\end{array}$ & $\begin{array}{c}\text { RV- } \\
\text { Dysfunktion im } \\
\text { Echo/CT }\end{array}$ & $\begin{array}{c}\text { kardiale } \\
\text { Biomarker } \uparrow\end{array}$ \\
\hline hoch & + & $(+)^{1}$ & + & $(+)^{1}$ \\
$\begin{array}{l}\text { intermediär-hoch } \\
\begin{array}{l}\text { intermediär- } \\
\text { niedrig }\end{array}\end{array}$ & - & + & + & + \\
niedrig & - & + & \multicolumn{2}{|c|}{$+/-2$} \\
\hline
\end{tabular}

${ }^{1}$ Bestimmung bei hämodynamisch instabilen Patienten nicht notwendig; ${ }^{2}$ 0-1 von beiden positiv;

${ }_{3}^{3}$ Bestimmung optional, bei durchgeführter Bestimmung beides negativ.

\subsection{Therapie der akuten Lungenembolie}

Die Therapie von Hochrisiko-Patienten erfolgt durch eine intravenöse (i.v.) Antikoagulation mit unfraktioniertem Heparin und reperfundierende Maßnahmen (Konstantinides et al. 2014). An erster Stelle steht hierbei die systemische Thrombolyse. Liegen bei dem Patienten absolute Kontraindikationen gegen eine Thrombolyse vor - wie beispielsweise ein hämorrhagischer Schlaganfall, ein schweres Trauma oder eine Operation in den letzten drei Wochen - oder ist die Thrombolyse nicht erfolgreich, so kann eine chirurgische Embolektomie oder eine interventionelle Thrombusfragmentierung bzw. eine Thrombektomie gewählt werden. Zusätzlich sollten intensivmedizinische Maßnahmen zur Unterstützung von Kreislauf und Atmung eingeleitet werden (Konstantinides et al. 2014).

Das therapeutische Vorgehen bei Nicht-Hochrisiko-LE-Patienten orientiert sich nach den ESC-Leitlinien 2014 an der durch den PESI/sPESI beeinflussten Risikoklassifikation: niedrig oder intermediär (Konstantinides et al. 2014). So können beispielsweise Patienten mit einem niedrigen klinischen Risiko für Komplikationen von einer frühzeitigen Entlassung profitieren, da ihre Mortalitätswahrscheinlichkeit bei <1\% liegt (Aujesky et al. 2005; Tamizifar et al. 2016). Besteht bei einem Patienten ein intermediär-hohes Risiko für schwerwiegende Komplikationen sollten eine Überwachung und bei hämodynamischer Verschlechterung reperfundierende Maßnahmen in Betracht gezogen werden (Jiménez et al. 2010b; Konstantinides et al. 2014). Liegt hingegen ein intermediär-niedriges Risiko vor, sind diese Maßnahmen nicht notwendig (Konstantinides et al. 2014). Therapeutisch erfolgt in jedem der Fälle eine subkutane (s.c.) Antikoagulation mit niedermolekularem Heparin oder Fondaparinux in gewichtsadaptierten Dosen. Bei hämodynamisch stabilen Patienten kann bereits in der Akutphase mit einem der beiden Nicht-Vitamin-K-abhängigen oralen Antikoagulanzien Apixaban oder Rivaroxaban begonnen werden (Konstantinides et al. 2014) (Abb. 4). 

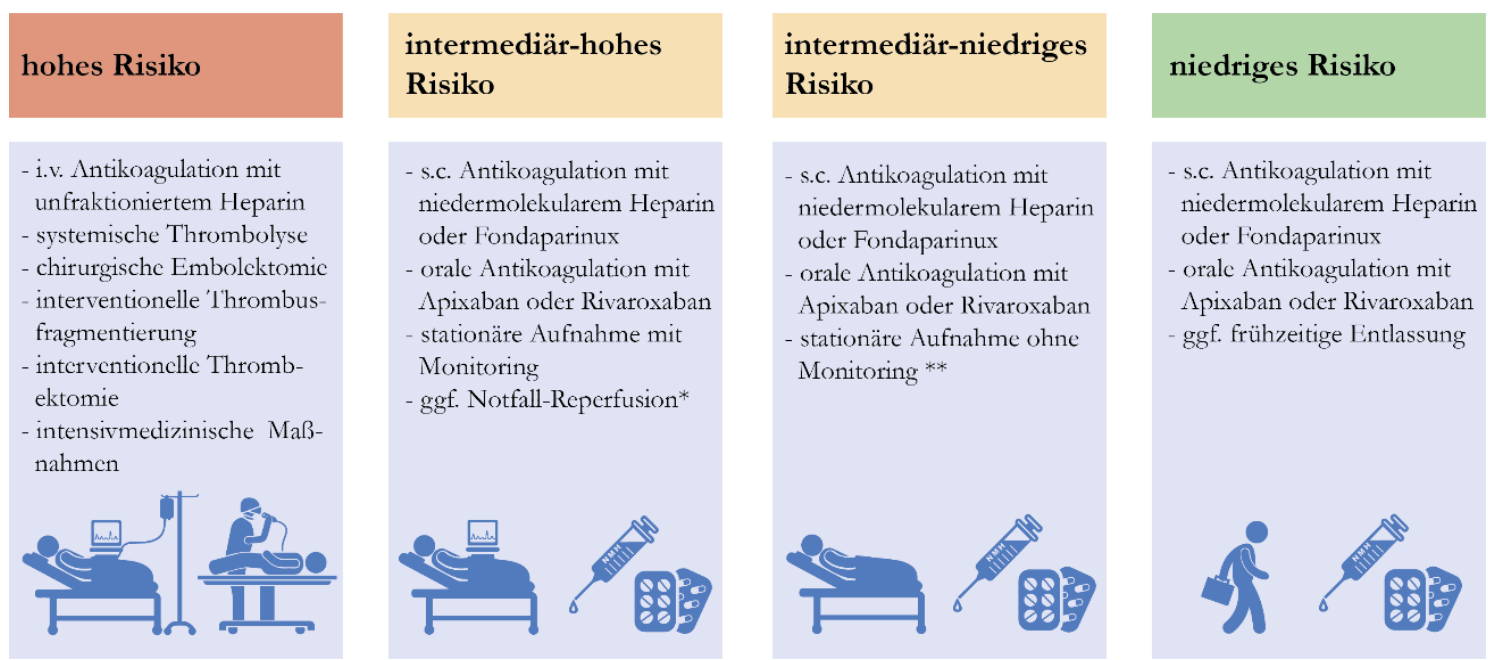

Abb. 4: Risikoadaptierte therapeutische Strategien der akuten LE.

* bei klinischen Zeichen einer hämodynamischen Dekompensation: Thrombolyse, chirurgische Embolektomie oder perkutane kathetergeführte Behandlung; ** bei positivem Troponin-Test: Monitoring in Erwägung ziehen, auch ohne Hinweise auf eine RV-Dysfunktion im Echo/CT.

\subsection{Die Computertomographie als Teil des diagnostischen Standard- algorithmus}

Heutzutage ist die Multidetektor-Computertomographie (MDCT) wegen ihrer hohen diagnostischen Genauigkeit (Meinel et al. 2015) das Mittel der Wahl zur Diagnose einer akuten LE (Becattini et al. 2014; Konstantinides et al. 2014; Goldhaber 2015). Sie besitzt eine hohe räumliche und zeitliche Auflösung sowie eine qualitativ hochwertige Darstellung der durch Thromben verursachten Kontrastmittelaussparung in arteriellen Gefäßen (Konstantinides et al. 2014). Mit ihrer Geschwindigkeit ist sie anderen bildgebenden Mitteln überlegen (Tapson 2008). Das CT kann in einem Großteil der Fälle als alleinige Bildgebung zur Diagnose oder zum Ausschluss einer akuten LE verwendet werden (Konstantinides 2008; Konstantinides et al. 2014). Seine Sensitivität beträgt 83\% und seine Spezifität 96\% (Stein et al. 2006). Der positive prädiktive Wert (PPW) liegt bei einer hohen Wahrscheinlichkeit für das Vorliegen einer akuten LE bei 96\%, bei einer mittleren Wahrscheinlichkeit bei $92 \%$ und bei 58\% im Falle einer niedrigen Wahrscheinlichkeit. Ein zusätzlich durchgeführtes CT der Venen zur Suche nach TVT erhöht die Sensitivität auf 90\% (Stein et al. 2006), jedoch wird diese Untersuchung aufgrund der erhöhten Strahlenexposition des CTs nicht mehr empfohlen. Die Multidetektor-CT weist einige Verbesserungen gegenüber der SingledetektorCT auf: verkürzte Scan-Zeiten, eine verminderte Schichtdicke (Tapson 2008) und eine bessere Darstellung von Segment- und Subsegmentgefäßen (Patel et al. 2003; Tapson 2008). Durch den Wechsel von $3 \mathrm{~mm}$ Schichtdicke zu $1 \mathrm{~mm}$ stieg die Entdeckungshäufigkeit sub- 
segmentaler Emboli um 40\% und die Zahl unentdeckter LE-Fälle fiel um 70\% (Schoepf et al. 2002).

\subsection{Thrombuslast in der MDCT-Angiographie}

Die Thrombuslast wird definiert als die Lokalisation der Thromben in den verschiedenen pulmonalarteriellen Gefäßästen oder als Abschätzung des prozentualen Gefäßverschlusses (Vedovati et al. 2012). Nachdem die Risikostratifizierung anhand des hämodynamischen Status die Bestimmung der Thrombuslast in der Pulmonalisangiographie weitgehend ersetzt hatte, ist das Interesse an dieser durch die weite Verbreitung der CT-Angiographie als diagnostisches Mittel erneut gestiegen (Vedovati et al. 2012; Vedovati et al. 2013). Thromben können in der MDCT zuverlässig bis auf die Segmentebene dargestellt werden und mit Hilfe von Kontrastmittel kann ebenfalls der Grad der Obstruktion festgestellt werden (Vedovati et al. 2013).

\subsubsection{Vorhersagekraft von Thromben in zentralen Gefäßen}

Zur einfachen Beurteilung der Thrombuslast im CT kommt z.B. die Lokalisationsmethode zum Einsatz. In einer Metaanalyse, in der nur proximal gelegene Emboli berücksichtigt wurden, waren nach Ausschluss zweier auf Krebspatienten spezialisierten Studien zentral gelegene Thromben bei normotensiven Patienten mit einem höheren Risiko für eine Dilatation des RV (Berghaus et al. 2010) und mit 2-fach erhöhtem Risiko für frühzeitiges Versterben assoziiert (Vedovati et al. 2012; Vedovati et al. 2013). Distale Emboli wiederum hatten ein relatives geringes Risiko für ungünstige Verläufe (Vedovati et al. 2012). Weiterführend wurde ermittelt, dass bilaterale Thromben nur in Kombination mit einer bestehenden RV-Dilatation als Risikofaktor galten (Vedovati et al. 2012). Gegensätzlich dazu wird argumentiert, dass nach Einschluss neuerer Studien und nach Anwendung strikterer Studienauswahlkriterien eine zentrale Thrombuslokalisation nicht mit einer erhöhten Mortalität verbunden sei. Die Schwere des LE-Ereignisses hänge demnach nicht nur von der Größe und Verteilung der Thromben ab, sondern auch vom kardiopulmonalen Krankheitsstatus des Patienten (Meinel et al. 2015). 


\subsubsection{Radiologische Obstruktionsindizes}

Bisherige Ansätze zur Erhebung der Thrombuslast greifen unter anderem auf die radiologischen Obstruktionsindices Qanadli-Score und Mastora-Score zurück (Tab. 3). Qanadli et al. entwickelten im Jahr 2001 einen Index, um die Obstruktion der Pulmonalgefäße und ihre Restperfusion in der nicht-invasiven CT-Diagnostik zu quantifizieren: jeder Lunge werden hierfür zehn Segmentarterien zugeordnet (drei im Oberlappen, zwei im Mittellappen und der Lingula, fünf im Unterlappen). Emboli in einer Segmentarterie zählen einen Punkt. Proximalen Arterien wird eine Punktzahl zugeordnet, die sich an der Anzahl der distal abzweigenden Segmentarterien orientiert (minimal ein Punkt, maximal 20 Punkte). Die verbleibende Perfusion distal des Embolus fließt via Multiplikation ebenfalls in die Bewertung ein: null Punkte zeigen das Fehlen eines Thrombus an, zwei Punkte die totale Okklusion. Der maximal erreichbare Punktwert des Qanadli-Scores liegt bei 40. Durch Errechnung des Quotienten aus persönlichem Wert und maximalem Punktwert und einer Multiplikation mit 100 ergibt sich der prozentuale Gefäßverschluss (Qanadli et al. 2001). Die Entwickler beschreiben ihren Score als Untersucher-unabhängig, simpel, reproduzierbar und auch bei anatomischen Variationen als einfach anwendbar (Qanadli et al. 2001). Ihre Auffassung wird in anderen Untersuchungen widerlegt (Vedovati et al. 2012; Vedovati et al. 2013): die absolute Anzahl der Thrombusverschlüsse, insbesondere die jeder Segmentarterie, sei schwierig zu kalkulieren und für die Auswertung würde ein Radiologe, der sich mit der Beurteilung von LEs auskenne, benötigt. Damit sei der Qanadli-Score für die notfallmäßige Risikostratifizierung ungeeignet (Vedovati et al. 2013). Des Weiteren wird kritisiert, es liege eine geringe Reproduzierbarkeit und eine hohe Interobserver-Variabilität vor (Vedovati et al. 2012). Der Mastora-Score wurde ebenfalls für die Nutzung auf CT-Bildern entwickelt (Engelke et al. 2006). Beurteilt werden hier fünf mediastinale, sechs Lappen- und 20 Segmentarterien. Der Grad des Gefäßverschlusses wird auf einer Skala von $1(<25 \%)$ bis 5 $(100 \%)$ angegeben, mit einem maximalen Obstruktionsindex von 155. Der persönliche Punktwert dividiert durch den maximalen Wert ergibt die prozentuale Gefäßverlegung (Mastora et al. 2003). Mastora et al. (2003) sehen ihren Score im Vergleich mit beispielsweise dem Qanadli-Score als präziser an. Als Limitation bei der Entwicklung ihres Scores nennen die Autoren die ausschließliche Verwendung optimaler CT-Bilder, weshalb der nicht unwesentliche Anteil suboptimaler Bildgebung vernachlässigt wurde (Mastora et al. 2003). Eine Studie von Engelke et al. (2006) befindet den Mastora-Score als Untersucherunabhängig und reproduzierbar und hebt positiv hervor, dass dieser Score im Gegensatz zu anderen Scores keine Schlüsse über die Thrombuslast peripherer Gefäße anhand der Thrombuslast proximaler Gefäßverschlüsse ziehe. Aufgrund seines hohen NPW von 0,96 
eignet sich der Mastora-Score insbesondere zur Identifikation von Patienten mit einem niedrigen Risiko für Komplikationen im Krankheitsverlauf (Engelke und Marten 2007). Kritikpunkte an beiden Scores sind die im Vergleich miteinander unterschiedlichen Ergebnisse beim gemittelten Prozentsatz des Gefäßverschlusses (Engelke et al. 2006) und die unterschiedliche Gewichtung der subsegmentalen Emboli (Furlan et al. 2012). Gegenüber Verschlüssen kleiner Arterien, z.B. durch Fettembolien oder durch andere nichtthrombotische Ursachen, weisen beide Obstruktionsindices eine geringe Sensitivität auf (Engelke und Marten 2007). Aufgrund ihrer Komplexität und abweichenden Untersuchungsergebnisse und der daraus resultierenden eingeschränkten Vorhersagekraft finden sie in der klinischen Routine der Risikostratifizierung bei akuter LE wenig Anwendung.

Tab. 3: Gegenüberstellung des Qanadli-Scores und des Mastora-Scores (modifiziert nach Qanadli et al. 2001; Mastora et al. 2003).

\begin{tabular}{|c|c|c|}
\hline & Qanadli-Score & Mastora-Score \\
\hline zugeordnete Arterienanzahl & $\begin{array}{l}10 \text { Segmentarterien/Lunge } \\
=20 \text { Segmentarterien }\end{array}$ & $\begin{array}{c}5 \text { mediastinale, } 6 \text { Lappen-, } \\
20 \text { Segmentarterien }\end{array}$ \\
\hline $\begin{array}{l}\text { Punktwertung einer verschlos- } \\
\text { senen proximalen Arterie }\end{array}$ & $\begin{array}{l}\text { anhand nachfolgender Seg- } \\
\text { mentarterienanzahl (max. 10) }\end{array}$ & $\begin{array}{c}1-5 \\
(<25 \%-100 \% \text { Verschluss })\end{array}$ \\
\hline $\begin{array}{l}\text { Punktwertung einer verschlos- } \\
\text { senen Segmentarterie }\end{array}$ & 1 & $\begin{array}{c}1-5 \\
(<25 \%-100 \% \text { Verschluss })\end{array}$ \\
\hline weitere Gewichtung & $\begin{array}{l}\text { Multiplikation mit 0-2 (voll- } \\
\text { ständige/partielle/keine ver- } \\
\text { bleibende distale Perfusion) }\end{array}$ & keine \\
\hline $\begin{array}{l}\text { maximal erreichbarer Punkt- } \\
\text { wert }\end{array}$ & 40 & 155 \\
\hline $\begin{array}{l}\text { Berechnung der prozentualen } \\
\text { Gefäßverlegung }\end{array}$ & \multicolumn{2}{|c|}{ (persönlicher Punktwert/maximaler Punktwert) x 100} \\
\hline
\end{tabular}

\subsubsection{Vorhersagekraft der Thrombusgröße}

Das Volumen des Thrombus korreliert stark mit den Scores zur Bestimmung der Thrombuslast und ist größer bei Patienten mit einer RV-Aufweitung (Furlan et al. 2012). Obstruktionsindices wie z.B. der von Mastora et al. entwickelte sind bei Patienten mit Vergrößerung des RV höher als bei solchen ohne, sie haben dennoch keine Vorhersagekraft bezüglich eines ungünstigen Ausgangs (Apfaltrer et al. 2012; Vedovati et al. 2012). Auch ein Qanadli-Score mit einem Punktwert $>18$ gilt als unabhängiger Prädiktor für eine RVDilatation (Rodrigues et al. 2012). Ein Mastora-Score über 21,3\% ist mit einem erhöhten Risiko für die Entstehung eines akuten Cor pulmonale assoziiert und mit einem 6,7-fach höheren Risiko für einen frühzeitigen Tod (Engelke et al. 2006). Diesem letzten Teil wird 
in neueren Studien widersprochen, die weder dem Mastora- noch dem Qanadli-Score eine prognostische Aussagekraft bezüglich der 30-Tages-Mortalität oder der 3-MonatsMortalität bescheinigen (Furlan et al. 2012; Vedovati et al. 2013).

\subsection{Bestimmung der rechtsventrikulären Dysfunktion}

Die Bestimmung der RV-Dysfunktion oder der myokardialen Schädigung kann mittels CT, TTE bzw. über die Messung kardialer Biomarker wie BNP, Troponin oder über neuere Marker wie H-FABP (Puls et al. 2007) erfolgen. Becattini et al. bemängeln in ihrer Untersuchung aus dem Jahre 2014, eine TTE, aber auch die Auswertung einiger Biomarker sei nicht in allen Krankenhäusern standardmäßig rund um die Uhr möglich. Des Weiteren fehle eine einheitliche Definition der RV-Dysfunktion (ten Wolde et al. 2004; Sanchez et al. 2008). Im Echo stehen Hypokinesie des RV, RV > LV oder ein rechtsventrikulärer enddiastolischer Diameter (RVEDD) $>30 \mathrm{~mm}$, Rückfluss über die Trikuspidalklappe und Verlust des inspiratorischen Kollapses der Vena cava inferior stellvertretend für diese Fehlfunktion (ten Wolde et al. 2004; Puls et al. 2007). Von den für die Vermessung des CTs zur Verfügung stehenden Methoden (diameter ratio, volume ratio, area ratio) ist die Messung der Right-to-Left Ventricular Ratio (RV/LV-Ratio) die am häufigsten verwendete, da sie einfach zu berechnen ist und dem Konzept der RV-Dilatation in der T'TE ähnelt (Becattini et al. 2014). Auch konnte gezeigt werden, dass eine zweidimensionale Messung einer dreidimensionalen Rekonstruktion des Ventrikels in ihrer prognostischen Aussagekraft nicht unterlegen ist (Lu et al. 2012).mast

\subsubsection{Aussagekraft der RV/LV-Ratio in der Computertomographie}

Die MDCT erlaubt die Darstellung und Vermessung der vier Herzkammern und kann damit eine Alternative zur TTE für die Bestimmung der RV-Dilatation im Sinne einer Dysfunktion sein (Becattini et al. 2011; Jiménez et al. 2014). Die RV/LV-Ratio im MDCT ist größer bei Patienten mit in der TTE nachgewiesener Dysfunktion des RV als bei solchen ohne (Becattini et al. 2011). Eine Fehlfunktion der rechten Herzkammer im CT ist ein unabhängiger Prädiktor für die Mortalität oder klinische Verschlechterung während des stationären Aufenthaltes, weshalb sie sich zur Risikostratifizierung eignet (Becattini et al. 2011). In ihrer Untersuchung legten die Autoren den Cut-off bei RV/LV $\geq 0,9$ fest. Die gute Korrelation zwischen der Bildauswertung durch einen Radiologen und der eines Arztes mit Routine in der Beurteilung von LE-CTs sprach für die Glaubwürdigkeit der durchgeführ- 
ten Messungen im MDCT (Becattini et al. 2011). Andere Untersuchungen hatten ähnliche Resultate: eine erhöhte RV/LV-Ratio im CT geht mit einem höheren Risiko für LEassoziierte Sterblichkeit innerhalb der ersten 30 Tage einher (Meinel et al. 2015). Kritik an der prognostischen Aussagekraft der RV/LV-Ratio im MDCT übt die Prognostic Value of CT Study (PROTECT) von Jiménez et al. (2014): es gebe zwar eine Assoziation zwischen einer vergrößerten RV/LV-Ratio und einer erhöhten Kurzzeit-Mortalität oder lebensbedrohlichen Verschlechterungen bei normotensiven Patienten, doch sei diese ohne statistische Signifikanz. Zusätzlich könne keine Identifizierung von low-risk Patienten und deren Prognose stattfinden (Coutance et al. 2011; Jiménez et al. 2014). Zur Definition einer vergrößerten RV/LV-Ratio fehle ferner ein einheitlicher Cut-off (Jiménez et al. 2014; Becattini et al. 2014); dieser wird zur Orientierung von den ESC-Leitlinien auf $\geq 0,9$ bzw. $\geq 1,0$ festgesetzt (Konstantinides et al. 2014). Der CT-Ansatz hat, im Gegensatz zur TTE, einige Limitationen bei der Darstellung der Funktionalität des RV: RV-Hypokinesie, pulmonale Hypertension und Trikuspidal-Beweglichkeit können nicht beurteilt werden (Becattini et al. 2011; Becattini et al. 2014). Zudem fehlt eine elektrokardiographische (EKG)-Triggerung (Hefeda und Elmasry 2014) und eine standardisierte achsengerechte Darstellung des Herzens im Programm der CT-LE-Diagnostik.

\subsection{Zielsetzung der Arbeit}

Die optimale Risikostratifizierung von Patienten mit einer akuten LE stellt weiterhin eine Herausforderung dar, weshalb in der vorliegenden Arbeit anhand von zweidimensional ausgewerteten CT-Bildern folgende Fragestellungen und Zielsetzungen untersucht wurden:

1) Die Entwicklung eines einfach anwendbaren und reproduzierbaren Scores zur Bestimmung der Thrombuslast (Thrombuslokalisation und Thrombusgröße) zentraler Gefäße und die Bestimmung ihres prognostischen Wertes für den klinischen Verlauf.

2) Die Ermittlung des Verhältnisses von RV zu LV und die Korrelation der RV/LVRatio mit dem klinischen Verlauf der Patienten.

3) Die Betrachtung der Aussagekraft der RV/LV-Ratio allein und in Kombination mit der Thrombuslokalisation und Thrombusgröße sowie weiterführend die Bestimmung des optimalen Cut-off-Wertes der RV/LV-Ratio für die Vorhersage schwerwiegender Komplikationen.

4) Die Eignung der identifizierten Parameter (aus 1-3) zur Risikostratifizierung in einem rein normotensiven Patientenkollektiv. 


\section{Material und Methoden}

\subsection{Studienprotokoll}

Im Zeitraum von Januar 2010 bis Mai 2016 wurden in der Universitätsmedizin Göttingen 488 Patienten mit der gesicherten Diagnose einer akuten LE in das Pulmonary Embolism Registry Göttingen (PERGO) eingeschlossen. Das PERGO ist eine prospektiv fortlaufende, nicht-interventionelle Kohortenstudie, die alle Patienten mit einer nach den jeweils gültigen Leitlinien (Torbicki et al. 2008; Konstantinides et al. 2014) diagnostizierten akuten LE erfasst. Die Ethikkommission der Universitätsmedizin Göttingen genehmigte das Studienprotokoll (Antragsnummer 14/6/10).

\subsubsection{Ein- und Ausschlusskriterien}

Zum Einschluss eines Patienten in das PERGO musste dieser mindestens 18 Jahre alt sein und an einer objektiv bestätigten akuten LE erkrankt sein. Für die Zustimmung zur Teilnahme an der Studie wurden der Patient oder sein gesetzlicher Vertreter von der Studienbetreuerin über den Ablauf, die Risiken und den Nutzen aufgeklärt (siehe Anhang 6.1). Von der Teilnahme ausgeschlossen wurden Patienten, die ihre Einwilligung nicht gaben oder diese widerriefen. Für den Einschluss in das Patientenkollektiv der vorliegenden Arbeit mussten zusätzlich zu den oben genannten Kriterien ein CT durchgeführt worden sein und die Bilder zur retrospektiven Auswertung in adäquater Qualität vorliegen.

\subsubsection{Studienablauf}

Für die Rekrutierung von Patienten mit dem Verdacht auf oder der bestätigten Diagnose einer akuten LE fand ein tägliches Screening der interdisziplinären Notaufnahme und der durchgeführten CTs sowie eine Kontaktierung der kardiologischen Stationen statt. Die Verdachtsdiagnose bestätigte und behandelte der betreuende Arzt anhand der aktuellen Leitlinien (Torbicki et al. 2008; Konstantinides et al. 2014). Das Studienprotokoll übte keinen Einfluss auf diesen diagnostischen Algorithmus, die Risikostratifizierung oder eine mögliche Therapie aus. Mittels eines standardisierten Anamnesebogens (siehe Anhang 6.2) und Akteneinsicht erhob die Studienbetreuerin Patienteninformationen wie Risikofaktoren, Symptomatik, Vormedikation und Vorerkrankungen und den klinischen Status bei Diagnosestellung. Diese wurden pseudonymisiert gespeichert. Während der Hospitalisierung oder 
innerhalb der ersten 30 Tage wurden zusätzlich noch eventuell auftretende Komplikationen vermerkt. Die Überlebensdauer von Patienten wurde entweder durch Akteneinsicht, im Falle eines Exitus letalis während des stationären Aufenthaltes oder durch Abfrage bei den zuständigen Einwohnermeldeämtern dokumentiert. Die Todesursache konnte mit Hilfe des Hausarztes ermittelt werden.

\subsubsection{Biomaterial und Laborparameter}

Zur Komplettierung der Patientenbefunde wurden venöse Blutproben vom Zeitpunkt der Diagnosestellung (tOh) für spätere Analysen aufbewahrt. Hierfür pipettierte die Studienbetreuerin $250 \mu \mathrm{l}$ Plasma von in der klinischen Routine abgenommenen Rückstellproben der Abteilung für Klinische Chemie der Universitätsmedizin Göttingen in Aliquots ab. Nach detaillierter Beschriftung der Proben wurden diese bei $-80^{\circ} \mathrm{C}$ eingefroren; die Aufbewahrungszeit beträgt zehn Jahre. Die Beurteilung der Laborparameter zum Zeitpunkt der Aufnahme erfolgte anhand von in der Literatur festgelegten Cut-off-Werten. Mittels der vereinfachten Modification of Diet in Renal Disease(MDRD)-Formel wurde die glomeruläre Filtrationsrate (GFR) bestimmt; der Cut-off des NT-proBNP bei $\geq 600 \mathrm{pg} / \mathrm{ml}$ entstammte einer Untersuchung von Lankeit et al. (2014). Für das hochsensitive Troponin T (hsTnT) konnte ein Wert $\geq 14 \mathrm{pg} / \mathrm{ml}$ als prognostisch relevant festgelegt werden (Lankeit et al. 2010; Lankeit et al. 2011).

\subsubsection{Definition der Endpunkte}

Tab. 4: Definition des primären und der sekundären Endpunkte (EP).

\begin{tabular}{|c|c|}
\hline Primärer EP & Sekundäre EP \\
\hline \multicolumn{2}{|l|}{ initiale kardiopulmonale Reanimation } \\
\hline \multicolumn{2}{|l|}{ kardiogener Schock } \\
\hline \multicolumn{2}{|c|}{ Komplikationen während des stationären Aufenthaltes oder innerhalb der ersten 30 Tage: } \\
\hline - Katecholaminpflichtigkeit & - endotracheale Intubation \\
\hline - kardiopulmonale Reanimation & $\begin{array}{l}\text { - Exitus letalis als Folge jeglicher Ursa- } \\
\text { che (LE-abhängig + LE-unabhängig) }\end{array}$ \\
\hline - Exitus letalis als Folge der akuten LE & \\
\hline
\end{tabular}




\subsection{Technische Aspekte der Computertomographie}

Im diagnostischen Algorithmus zur Diagnosesicherung einer akuten LE ist heutzutage die kontrastmittelgestützte MDCT-Angiographie verankert (Konstantinides et al. 2014). Die MDCT erfolgte an CT-Scannern der Klasse Somatom Definition Flash (64 Zeiler; 128 Zeiler), Somatom Definition AS+ (64 Zeiler; 128 Zeiler) oder Somatom Sensation 16 (16 Zeiler) (alle Siemens Healthcare, Erlangen). Bei Aufnahme der Bilder betrug die Schichtdicke $5 \mathrm{~mm}$, diese wurde für die multiplanare Rekonstruktion im Weichteil-, Lungen-, Knochenalgorithmus auf $1 \mathrm{~mm}$ reduziert. Dem Standardprotokoll der CT-Angiographie gemäß erfolgte die Gabe von 45-70 ml Iomeprol (Imeron 350 (350 mg Iod/ml), Bracco Imaging Deutschland GmbH, Konstanz) i.v. mit einer Geschwindigkeit von $4 \mathrm{ml} / \mathrm{s}$; die Bildaufnahme fand in der früharteriellen Phase, das heißt bei einem deutlichen Kontrastmittelbolus in der Arteria pulmonalis, statt. Digital archiviert wurden die Bilder im Picture Archiving and Communication System (PACS).

Von den 488 im Untersuchungszeitraum aufgenommenen Patienten mussten 24 Patienten aus dem in der vorliegenden Arbeit beurteilten Patientenkollektiv ausgeschlossen werden, da kein CT stattfand. Weitere elf erhielten zwar zur Diagnosesicherung ein CT, doch stand dieses für die retrospektive Auswertung nicht zur Verfügung, da die MDCT beispielsweise in externen Krankenhäusern erfolgte. Ein weiteres Ausschlusskriterium aus dem Untersuchungskollektiv war eine mangelhafte CT-Qualität aufgrund derer keine adäquate retrospektive Beurteilung des Bildes hinsichtlich der Ventrikelgröße und der zentralen Pulmonalarterien stattfinden konnte. Die fehlende Verwendung von Kontrastmittel, eine zu späte Bildaufnahme nach Kontrastmittelgabe oder der Zufallsbefund einer akuten LE während einer anderen CT-Untersuchung und damit einhergehend die suboptimale Darstellung der Pulmonalarterien waren ursächlich für die insuffiziente Qualität. Dies traf bei elf weiteren Patienten zu (Abb. 5). Die Gesamtanzahl der retrospektiv untersuchten und ausgewerteten CT-Bilder betrug damit 442. 
Patienten mit bestätigter Diagnose einer akuten Lungenembolie: 488

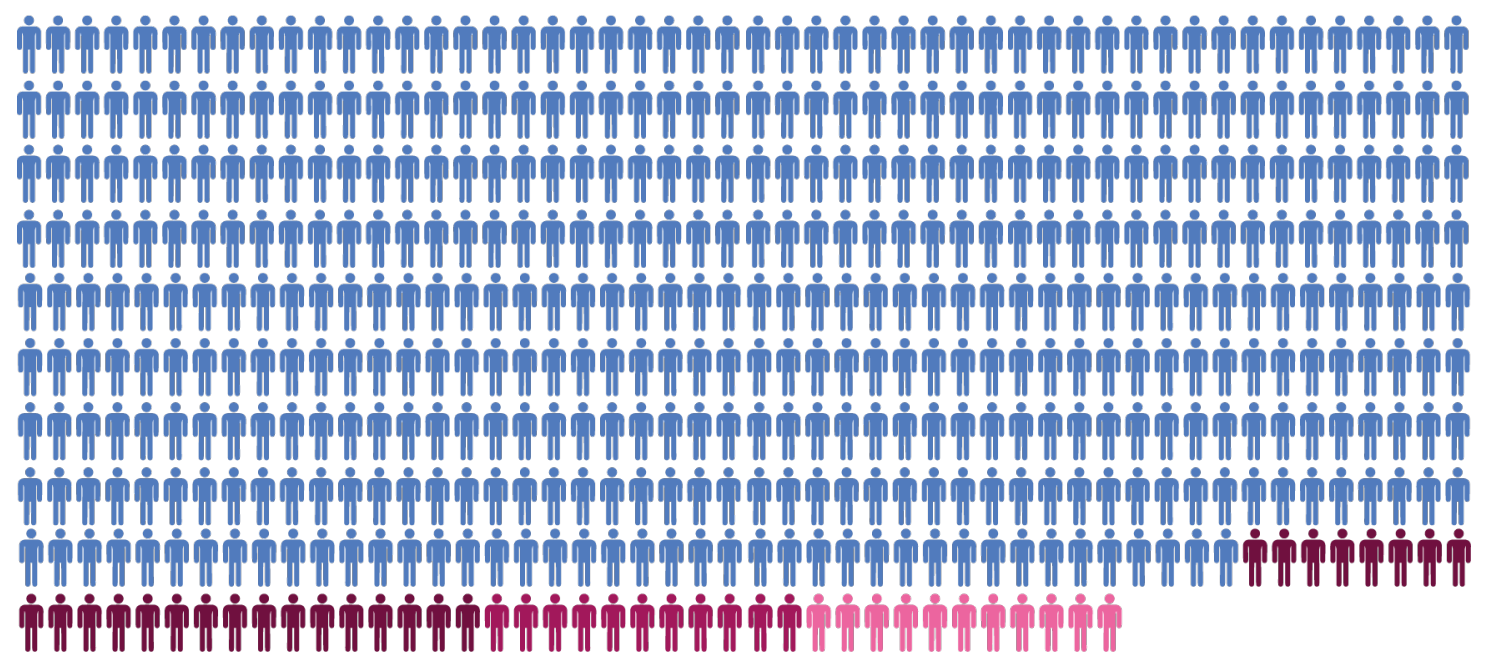

kein CT durchgeführt: 24

keine CT-Bilder verfügbar: 11

mangelhafte CT-Bildqualität: 11

ausgewertete C'T-Bilder: 442

Abb. 5: Gründe für den Ausschluss von Patienten aus der retrospektiven CT-Auswertung.

\subsection{Durchführung der computertomographischen Auswertung}

Prof. Dr. Claudia Dellas (Untersucher 2; U2) (Klinik für Pädiatrische Kardiologie und Intensivmedizin, Universitätsmedizin Göttingen), Dr. Kristian Hellenkamp (Untersucher 3; U3) und Judith Reuter (Untersucher 1; U1) (beide Klinik für Kardiologie und Pneumologie, Universitätsmedizin Göttingen) werteten die CT-Bilder des 442 Patienten umfassenden Kollektivs aus. Die Post-hoc-Analyse der kontrastmittelgestützten MDCT wurde mittels der Centricity Enterprise Web V3.0 Software von GE Medical Systems, France, durchgeführt. Für jeden Patienten fand die Beurteilung hinsichtlich Thrombuslokalisation, prozentualem Gefäßverschluss und Messung der RV/LV-Ratio durch zwei der drei beteiligten Untersucher statt. Zum Zeitpunkt der Auswertung waren allen Untersuchern die echokardiographischen Ergebnisse, der klinische Verlauf des Patienten und die Ergebnisse der KoUntersucher unbekannt. Bei Diskrepanzen hinsichtlich der Thrombusverteilung wurden diese mit allen drei Untersuchern besprochen und im Konsens aufgelöst. Unterschiede in Messungen der Thrombuslast in Prozent und der RV/LV-Ratio wurden für die weitere statistische Auswertung gemittelt. 


\subsubsection{Thrombuslast-Messung}

Für die Bestimmung der Thrombuslast wurden ausschließlich der Pulmonalarterienhauptstamm (HS) sowie die rechte (RPA) und linke (LPA) Pulmonalarterie in der Transversalebene der Bildansicht des Weichteilfensters beurteilt. Befand sich ein Thrombus in einem oder mehreren dieser drei Gefäße, so wurde sein Durchmesser (in mm) an der Stelle mit der größten Reduktion des durchflossenen Lumens im zweidimensionalen Bild vermessen (Abb. 6a). Aus dem Quotienten dieses Wertes (in $\mathrm{mm}$ ) und des an derselben Stelle gemessenen Gefäßquerschnitts (in mm) und einer Multiplikation mit dem Faktor 100 ließ sich die prozentuale Arterienverlegung ermitteln:

\section{I (in mm) / II (in mm) $* 100=$ Arterienverlegung (in \%)}

In der internen Codierung wurde ein auf der Lungenarterienbifurkation reitender Thrombus als in allen drei Gefäßen befindlich gewertet (Abb. 6b). Die Vermessung der Thrombusgröße im HS und des Gefäßquerschnitts an gleicher Stelle waren aufgrund dieser speziellen reitenden Lage nicht möglich. Es fand in diesem Fall deshalb lediglich die Ermittlung der Verlegung von RPA und LPA statt.

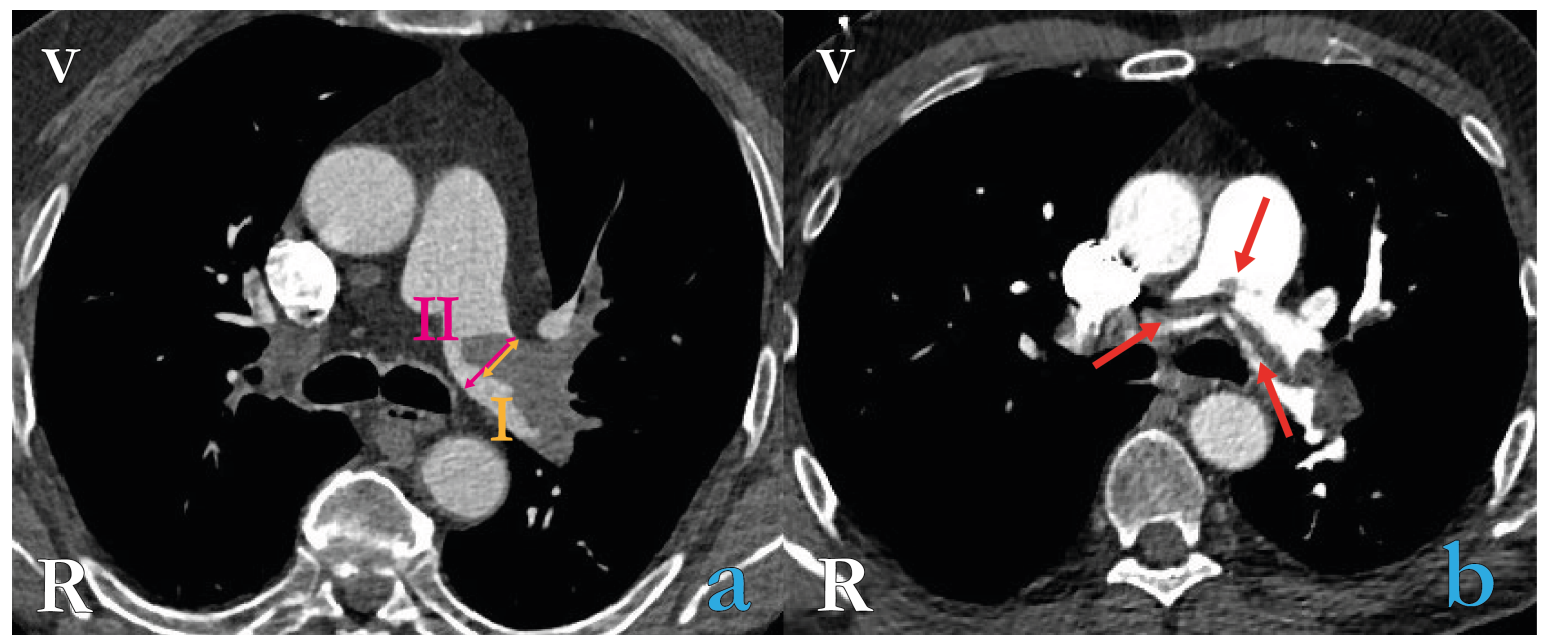

$\mathrm{v}=$ ventral, $\mathrm{R}=$ rechts; orange Linie $(\mathrm{I})=$ Thrombusdurchmesser, pinke Linie (II) = Gefäßdurchmesser, rote Pfeile $=$ reitender Thrombus.

Abb. 6 a: Vermessung des Thrombus- und Gefäßquerschnitts zur Bestimmung des prozentualen Gefäßverschlusses. b: Reitender Thrombus im HS. 


\subsubsection{Bestimmung der RV/LV-Ratio}

Die in der vorliegenden Arbeit angewandte Vermessung des Diameters des RV und LV in der zweidimensionalen Darstellung des CT ist in der Literatur oft beschrieben worden (Becattini et al. 2011; Jiménez et al. 2014). Hierbei wurde im Vier-Kammer-Blick in der Transversalebene des Weichteilfensters der maximale Abstand von ventrikulärem Endokard und interventrikulärem Septum, orthogonal zur langen Achse des Herzens, als Diameter (in $\mathrm{mm}$ ) der jeweiligen Herzkammer festgelegt (Abb. 7a). Die maximale Ventrikeldimension konnte dabei für den RV und für den LV in verschiedenen Schnittebenen gemessen werden. Aus dem Quotienten des RV-Durchmessers und des LV-Durchmessers ergab sich die RV/LV-Ratio (Klok et al. 2010; Becattini et al. 2011; Henzler et al. 2012). Abb. 7b zeigt beispielhaft die Vergrößerung des RV gegenüber des LV.

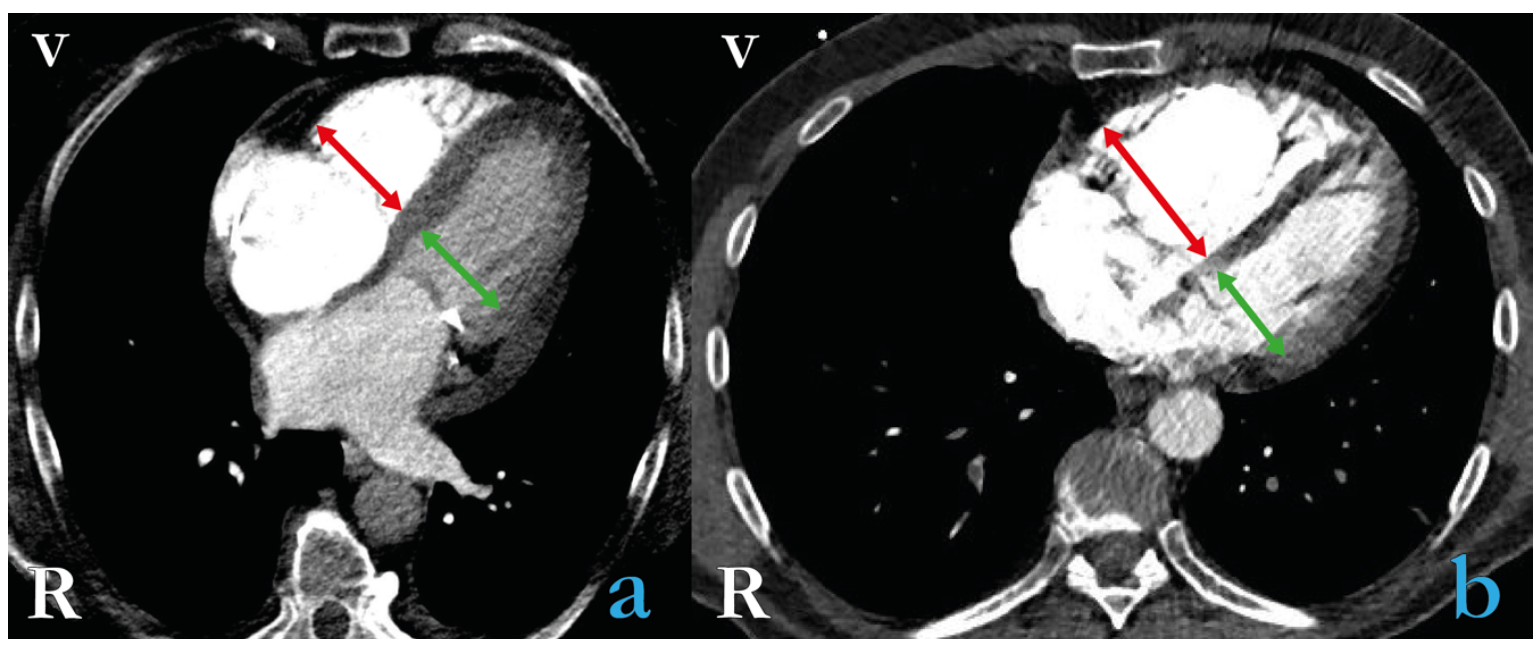

$\mathrm{v}=$ ventral, $\mathrm{R}=$ rechts; rote Linie $=\mathrm{RV}$, grüne Linie $=\mathrm{LV}$.

Abb. 7 a: Bestimmung des RV- und LV-Diameters. b: RV-Dilatation (RV $>$ LV).

\subsection{Statistik}

Die statistischen Auswertungen wurden mithilfe der Statistik Software SPSS Statistics Version 24 von IBM, Chicago, Illinois, durchgeführt. Bei kategorialen Variablen fand eine Bestimmung der Häufigkeiten innerhalb des jeweiligen Untersuchungskollektivs mit Angabe des dazugehörigen Prozentwertes statt, die Prüfung der Signifikanz dieser Ergebnisse wurde je nach Subgruppengröße mittels Fisher's Exact Test oder Chi-Quadrat-Test durchgeführt. Zur Überprüfung, ob die kontinuierlichen Variablen normalverteilt waren, wurde der Kolmogorov-Smirnov-Test angewandt. Das Ergebnis dieser Untersuchung war negativ. Aufgrund dessen wurden der Median und die zugehörigen 25. und 75. Quartile (Angabe als 
Interquartilsabstand (IQR)) bestimmt sowie die Signifikanz mittels Mann-Whitney U Test geprüft. Zur Überprüfung der Inter- und Intraobserver-Variabilität wurden zuerst die Mittelwerte der Abweichungen mit zugehöriger Standardabweichung sowie die Anzahl der Ausreißer bestimmt und für kontinuierliche Variablen der Intraclass-Korrelationskoeffizient (ICC) sowie für nominale Variablen der Kappa-Koeffizient (א) für alle drei Untersucherpaare ermittelt. Anschließend wurden die Ergebnisse aller drei Auswerter für eine BlandAltmann-Analyse zu zwei Messwerterhebungen zusammengefasst und bei dieser hinsichtlich systematischer Fehler, Streuung der Differenzen und individueller Abweichungsmuster beurteilt. Die Berechnung der Area Under the Curve (AUC) der RV/LV-Ratio bezogen auf die Thrombuslast und den primären EP der Studie fand in Receiver-Operating-Characteristics(ROC)-Analysen statt und wurde für das Gesamtkollektiv und die Subgruppe der normotensiven Patienten durchgeführt. Der optimale Cut-off-Wert der RV/LV-Ratio konnte durch Anwendung des Youden-Index bestimmt werden. Er lag in der vorliegenden Arbeit bei RV/LV >1,3. Zusätzlich wurden für diesen Cut-off die Sensitivität, Spezifität sowie PPW und NPW ermittelt. Für die Thrombuslast allein und für die Kombination eines Thrombus in HS + RPA + LPA mit dem zuvor bestimmten optimalen Cut-off der RV/LV-Ratio >1,3 fand ebenfalls eine Bestimmung der Sensitivität und Spezifität statt. Univariable logistische Regression wurde zur Bestimmung der prognostischen Relevanz von RV/LV-Ratio und Thrombuslast für das Erreichen des primären EP eingesetzt und als OR mit korrespondierendem 95\%-Konfidenzintervall (KI) präsentiert. Bei allen durchgeführten Tests war das Signifikanzniveau $\alpha=0,05$ und die Signifikanzprüfung zweiseitig. 


\section{Ergebnisse}

\subsection{Beschreibung von Studienpopulation und primärem Endpunkt}

Die 442 Patienten umfassende Studienpopulation war zum Zeitpunkt der Aufnahme durchschnittlich 70 Jahre alt und bestand aus 212 Männern (48,0\%) und 230 Frauen (52,0\%). Häufig lagen eine arterielle Hypertonie (64,5\%), Hyperlipoproteinämie (22,4\%), koronare Herzerkrankungen (KHK) (18,3\%), chronische Herzinsuffizienz (17,0\%) oder Diabetes mellitus (17,0\%) als Begleiterkrankungen vor. Bei 38,0\% des Patientenkollektivs galt die akute LE als „provoziert“. Für den Großteil stellte die akute LE ein Erstereignis dar, bei $11,2 \%$ trat sie als Rezidiv auf. Eine maligne Grunderkrankung war bei 17,0\% der Patienten vorhanden. Besonders oft gaben die Patienten zum Zeitpunkt der Aufnahme Dyspnoe $(84,5 \%)$ und Thoraxschmerzen $(49,5 \%)$ an. Des Weiteren präsentierten sie sich klinisch häufig tachykard (37,2\%), hypoxisch (30,2\%) sowie mit Beinschmerzen oder Beinschwellung als Zeichen einer TVT (26,6\%). Seltener kamen Synkopen (13,6\%), Hypotonie $(11,2 \%)$ oder Hämoptysen (3,4\%) vor. Im Aufnahmelabor waren 65,2\% der bestimmten hsTnT-Werte und 55,1\% der gemessenen NT-proBNP-Werte pathologisch erhöht; die GFR war in 36,0\% der Fälle unterhalb der Norm im Sinne einer eingeschränkten Nierenfunktion. Die Anwendung der ESC-2014-Risikoklassifikation ordnete 14,0\% der Patienten der Gruppe mit niedrigem Risiko zum Aufnahmezeitpunkt zu. Ein intermediäres Risiko war bei 78,1\% (intermediär-niedrig 49,1\%, intermediär-hoch 29,0\%) und ein hohes Risiko bei 7,9\% der Patienten gegeben (Tab. 5). Grundlage der Zuordnung waren in der vorliegenden Arbeit - je nach Vorhandensein - die Echo- oder alternativ die CT-Befunde des RV und der sPESI.

Von den 442 Patienten des Kollektivs erreichten 58 (13,1\%) den primären EP bei Aufnahme, während des stationären Aufenthaltes oder innerhalb der ersten 30 Tage nach Diagnosestellung. Bei Betrachtung der einzelnen Komponenten waren 9,0\% der Patienten katecholaminpflichtig, 7,0\% litten an einem kardiogenen Schock zum Zeitpunkt der Aufnahme, 5,7\% wurden initial oder im Verlauf reanimationspflichtig und 4,5\% erlebten einen Exitus letalis als Folge der akuten LE. Wurde die klinische Präsentation bei Aufnahme auBen vor gelassen und nur die Komplikationen ab dem Zeitpunkt des stationären Aufenthaltes bewertet, so traten diese bei 12,9\% auf. Hierunter fielen alle Todesfälle (in Folge der akuten LE und in Folge anderer Ursachen) (6,6\%) sowie die endotracheale Intubation (7,7\%). Beides stellte einen sekundären EP dar (Tab. 6). Patienten, die den primären EP erreichten, hatten in der Anamnese öfter eine chronische Herzinsuffizienz oder eine Nie- 
reninsuffizienz, wiesen jedoch seltener eine LE in der Vorgeschichte auf. Häufige Symptome waren Synkopen, Hypotonie und Hypoxie (Tab. 5).

Tab. 5: Charakteristika, Begleiterkrankungen, Risikofaktoren, klinische Präsentationen, ausgewählte Laborparameter und Risikoklassifikationen der Studienpopulation bei Aufnahme und bei Erreichen des primären EP.

\begin{tabular}{|c|c|c|c|c|}
\hline Parameter & $\begin{array}{l}\text { Studien- } \\
\text { population } \\
(\mathrm{n}=442)\end{array}$ & $\begin{array}{l}\text { EP nicht } \\
\text { erreicht } \\
(n=384)\end{array}$ & $\begin{array}{l}\text { EP erreicht } \\
\quad(n=58)\end{array}$ & $\begin{array}{c}\text { p- } \\
\text { Wert }\end{array}$ \\
\hline Alter in Jahren & $70,0[56,0-78,0]$ & $72,0[57,3-82,0]$ & $74,5[59,5-81,8]$ & 0,334 \\
\hline $\begin{array}{l}\text { Geschlecht männlich } \\
\text { weiblich }\end{array}$ & $\begin{array}{l}212(48,0 \%) \\
230(52,0 \%)\end{array}$ & $\begin{array}{l}183(47,7 \%) \\
201(52,3 \%)\end{array}$ & $\begin{array}{l}29(50,0 \%) \\
29(50,0 \%)\end{array}$ & 0,779 \\
\hline $\mathrm{BMI}$ in $\mathrm{kg} / \mathrm{m}^{2}$ & $27,7[24,5-31,0]$ & $27,5[24,7-30,6]$ & $30,0[25,1-33,5]$ & 0,930 \\
\hline \multicolumn{5}{|c|}{ Begleiterkrankungen und kardiovaskuläre Risikofaktoren } \\
\hline chron. Herzinsuffizienz & $75(17,0 \%)$ & $59(15,4 \%)$ & $16(27,6 \%)$ & 0,037 \\
\hline KHK & $81(18,3 \%)$ & $67(17,4 \%)$ & $14(24,1 \%)$ & 0,273 \\
\hline COPD & $40(9,0 \%)$ & $32(8,3 \%)$ & $8(13,8 \%)$ & 0,215 \\
\hline arterielle Hypertonie & $285(64,5 \%)$ & $242(63,0 \%)$ & $43(74,1 \%)$ & 0,107 \\
\hline Hyperlipoproteinämie & $99(22,4 \%)$ & $89(23,2 \%)$ & $10(17,2 \%)$ & 0,398 \\
\hline Diabetes mellitus & $75(17,0 \%)$ & $62(16,1 \%)$ & $13(22,4 \%)$ & 0,260 \\
\hline Niereninsuffizienz & $61(13,8 \%)$ & $45(11,7 \%)$ & $16(27,6 \%)$ & 0,003 \\
\hline \multicolumn{5}{|c|}{ VTE-spezifische Risikofaktoren } \\
\hline provozierte VTE ${ }^{1}$ & $168(38,0 \%)$ & $144(37,5 \%)$ & $24(41,4 \%)$ & 0,565 \\
\hline vorherige LE & $49 / 440(11,2 \%)$ & $48 / 383(12,5 \%)$ & $1 / 57(1,8 \%)$ & 0,012 \\
\hline Z.n. Operation ${ }^{2}$ & $87(19,7 \%)$ & $72(18,8 \%)$ & $15(25,9 \%)$ & 0,216 \\
\hline Z.n. Trauma ${ }^{2}$ & $10(2,3 \%)$ & $8(2,1 \%)$ & $2(3,4 \%)$ & 0,627 \\
\hline maligner Tumor ${ }^{3}$ & $75(17,0 \%)$ & $67(17,4 \%)$ & $8(13,8 \%)$ & 0,577 \\
\hline Kontrazeptiva/ & $29(6,6 \%)$ & $27(7,0 \%)$ & $2(3,4 \%)$ & 0,404 \\
\hline Hormonpräparate & $26 / 230(11,3 \%)^{*}$ & $25 / 201(12,4 \%)^{*}$ & $1 / 29(3,4 \%)^{*}$ & $0,229^{*}$ \\
\hline Rauchen & $102 / 431(23,7 \%)$ & $88 / 381(23,1 \%)$ & $14 / 50(28,0 \%)$ & 0,479 \\
\hline Immobilisation 2,4 & $83 / 441(18,8 \%)$ & $68(17,7 \%)$ & $15(26,3 \%)$ & 0,145 \\
\hline \multicolumn{5}{|c|}{ Klinische Präsentation bei Aufnahme } \\
\hline Thoraxschmerz & $217 / 438(49,5 \%)$ & $203 / 383(53,0 \%)$ & $14 / 55(25,5 \%)$ & $<0,001$ \\
\hline Dyspnoe & $370 / 438(84,5 \%)$ & $327 / 383(85,4 \%)$ & $43 / 55(78,2 \%)$ & 0,167 \\
\hline Hämoptyse & $15 / 440(3,4 \%)$ & $14(3,6 \%)$ & $1 / 56(1,8 \%)$ & 0,705 \\
\hline Synkope & $60 / 441(13,6 \%)$ & $38(9,9 \%)$ & $22 / 57(38,6 \%)$ & $<0,001$ \\
\hline Zeichen einer TVT & $116 / 436(26,6 \%)$ & $104 / 381(27,3 \%)$ & $12 / 55(21,8 \%)$ & 0,514 \\
\hline Herzfrequenz in min $^{-1}$ & $90,0[75,0-105,0]$ & $95,0[76,5-110,0]$ & $\begin{array}{c}100,5[66,3- \\
120,8] \\
\end{array}$ & 0,034 \\
\hline $\begin{array}{l}\text { systolischer Blutdruck in } \\
\mathrm{mmHg}\end{array}$ & $\begin{array}{c}130,0[118,3- \\
150,0]\end{array}$ & $\begin{array}{c}130,0[114,5- \\
150,0]\end{array}$ & $\begin{array}{c}105,0[84,5- \\
136,5]\end{array}$ & $<0,001$ \\
\hline Atemfrequenz in min $^{-1}$ & $17,5[14,0-22,0]$ & $17,0[15,0-20,0]$ & $19[12,0-26,5]$ & 0,545 \\
\hline Tachykardie 5 & $159 / 427(37,2 \%)$ & $134 / 376(35,6 \%)$ & $25 / 51(49,0 \%)$ & 0,089 \\
\hline Hypotonie ${ }^{6}$ & $47 / 420(11,2 \%)$ & $23 / 374(6,1 \%)$ & $24 / 46(52,2 \%)$ & $<0,001$ \\
\hline Hypoxie $^{7}$ & $114 / 378(30,2 \%)$ & $81 / 325(24,9 \%)$ & $33 / 53(62,3 \%)$ & $<0,001$ \\
\hline
\end{tabular}




\begin{tabular}{|c|c|c|c|c|}
\hline \multicolumn{5}{|c|}{ Laborparameter bei Aufnahme } \\
\hline hsTnT in $\mathrm{pg} / \mathrm{ml}$ & $27,0[9,9-65,8]$ & $22,1[8,4-56,9]$ & $55,6[29,4-87,7]$ & $<0,001$ \\
\hline NT-proBNP in pg/ml & $\begin{array}{c}816,3[161,4- \\
2796,0]\end{array}$ & $\begin{array}{c}630,2[117,3- \\
2506,0]\end{array}$ & $\begin{array}{c}2289,0[739,2- \\
3884,0]\end{array}$ & $<0,001$ \\
\hline GFR in $\mathrm{ml} / \mathrm{min}$ & $69,9[51,7-88,6]$ & $73,3[53,9-86,5]$ & $38,7[32,3-67,4]$ & $<0,001$ \\
\hline hsTnT $\geq 14 \mathrm{pg} / \mathrm{ml}$ & $242 / 371(65,2 \%)$ & $203 / 330(61,5 \%)$ & $39 / 41(95,1 \%)$ & $<0,001$ \\
\hline NT-proBNP $\geq 600 \mathrm{pg} / \mathrm{ml}$ & $199 / 361(55,1 \%)$ & $166 / 319(52,0 \%)$ & $33 / 42(78,6 \%)$ & 0,001 \\
\hline GFR $<60 \mathrm{ml} / \mathrm{min} / 1,73 \mathrm{~m}^{2}$ & $154 / 428(36,0 \%)$ & $119 / 373(31,9 \%)$ & $35 / 55(63,6 \%)$ & $<0,001$ \\
\hline \multicolumn{5}{|l|}{ Risikoklassifikation } \\
\hline $\begin{array}{l}\text { PESI Risikoklassen: } \\
\text { niedrig (Klasse I-II) } \\
\text { intermediär bis hoch } \\
\text { (Klasse III-IV) } \\
\end{array}$ & $\begin{array}{l}178 / 440(40,5 \%) \\
262 / 440(59,5 \%)\end{array}$ & $\begin{array}{l}169 / 382(44,2 \%) \\
213 / 382(55,8 \%)\end{array}$ & $\begin{array}{c}9(15,5 \%) \\
49(84,5 \%)\end{array}$ & $<0,001$ \\
\hline sPESI Score $\geq 1$ Punkt & $304(68,6 \%)$ & $249(64,8 \%)$ & $55(94,8 \%)$ & $<0,001$ \\
\hline $\begin{array}{l}\text { ESC-2014-Risikoklassen: } \\
\text { niedrig } \\
\text { intermediär-niedrig } \\
\text { intermediär-hoch } \\
\text { hoch }\end{array}$ & $\begin{array}{c}62(14,0 \%) \\
217(49,1 \%) \\
128(29,0 \%) \\
35(7,9 \%)\end{array}$ & $\begin{array}{c}61(15,9 \%) \\
204(53,1 \%) \\
118(30,7 \%) \\
1(0,4 \%)\end{array}$ & $\begin{array}{c}1(1,7 \%) \\
13(22,4 \%) \\
10(17,2 \%) \\
34(58,6 \%)\end{array}$ & $<0,001$ \\
\hline
\end{tabular}

Angabe der kategorialen Variablen als Anzahl n der Patienten des jeweiligen Patientenkollektivs $\mathrm{n}=442, \mathrm{n}=384$ bzw. $\mathrm{n}=58$ mit dem korrespondierenden Prozentwert in Klammern. Bei unvollständiger Datenerhebung bezieht sich der Prozentwert auf die jeweils verfügbare Patientenzahl /n; Angabe der kontinuierlichen Variablen als Median mit dazugehörigem IQR in eckigen Klammern. 1 definiert als: Operation, Trauma, Immobilisation, Schwangerschaft, Kontrazeptiva/Hormontherapie innerhalb der letzten drei Monate; ${ }^{2}$ innerhalb der letzten vier Wochen; ${ }^{3}$ aktiv oder in Behandlung innerhalb der letzten sechs Monate; ${ }^{4}$ länger als drei Tage; ${ }^{5}$ Herzfrequenz $\geq 100 \mathrm{~min}^{-1}$; ${ }^{6}$ systolischer Blutdruck $<100 \mathrm{mmHg} ;{ }^{7} \mathrm{SaO}_{2}<90 \%$ oder $\mathrm{paO}_{2}<60 \mathrm{mmHg}$ mit oder ohne Sauerstoffgabe.

* Anzahl und Prozentwert bezogen auf die weiblichen Patientinnen des Kollektivs.

Tab. 6: Häufigkeiten der EP und der Komplikationen innerhalb der ersten 30 Tage.

\begin{tabular}{|l|c|}
\hline \multicolumn{1}{|c|}{ Endpunkte } & Häufigkeit \\
\hline primärer EP $*$ & $58(13,1 \%)$ \\
Katecholamine & $40(9,0 \%)$ \\
kardiogener Schock bei Aufnahme & $31 / 441(7,0 \%)$ \\
Reanimationspflichtigkeit (bei Aufnahme und/oder im Verlauf) & $25 / 441(5,7 \%)$ \\
Tod als Folge der LE & $20(4,5 \%)$ \\
Komplikationen im Verlauf** & $57(12,9 \%)$ \\
Tod jeglicher Ursache (LE-abhängig + LE-unabhängig) & $29(6,6 \%)$ \\
endotracheale Intubation & $34(7,7 \%)$ \\
\hline
\end{tabular}

Angabe der Häufigkeit als Anzahl $\mathrm{n}$ der Patienten des Gesamtkollektivs n=442 mit dem korrespondierenden Prozentwert in Klammern. Bei unvollständiger Datenerhebung bezieht sich der Prozentwert auf die jeweils verfügbare Patientenzahl $/ \mathrm{n}$.

* definiert als Katecholamine, kardiogener Schock bei Aufnahme, Reanimation bei Aufnahme und/ oder im Verlauf, Tod durch LE innerhalb der ersten 30 Tage, ${ }^{* *}$ definiert als Katecholamine, Reanimation im Verlauf, Tod jeglicher Ursache innerhalb der ersten 30 Tage, Intubation. 


\subsection{Interobserver- und Intraobserver-Variabilität}

In der Interobserver-Variabilität wurde untersucht, inwieweit die Ergebnisse vom Untersucher abhängig waren oder durch ihn beeinflusst wurden bzw. wie objektiv und unabhängig die Messungen waren. Die Analyse der 442 CT-Bilder hinsichtlich Thrombuslast und RV/LV-Ratio fand durch jeweils zwei der drei im Methodenteil unter 2.3 benannten Untersucher (U1, U2, U3) statt (Tab. 7). Zum Zeitpunkt der Auswertung waren allen Untersuchern die Ergebnisse der Ko-Untersucher, weiterführende Echo-Untersuchungsergebnisse und der klinische Verlauf des Patienten unbekannt.

Tab. 7: Gesamte und gemeinsame CT-Auswertungen der Untersucher.

\begin{tabular}{|c|c|c|c|}
\hline Untersucher & Auswertung insgesamt & gemeinsam mit U3 & gemeinsam mit U2 \\
\hline U1 & 269 & 204 & 65 \\
\hline U2 & 238 & 173 & \multicolumn{1}{|c}{} \\
\hline U3 & 377 & \multicolumn{2}{|l}{} \\
\cline { 1 - 3 } & &
\end{tabular}

\subsubsection{Interobserver-Vergleich und -Korrelation}

Die von den Untersuchern durchgeführten Messungen der prozentualen Gefäßverlegung von LPA und RPA sowie die Ermittlung der RV/LV-Ratio wurden mit Blick auf die mittlere Abweichung (in \%) und starke Ausreißer, das heißt Messungen $>100 \%$ Unterschied, verglichen (Tab. 8). Die gesamte Thrombuslast wurde hierbei definiert als Mittelwert des Gefäßverschlusses von LPA + RPA; ein zusätzlicher reitender Thrombus im HS wurde als vorhanden gewertet, seine Größe konnte jedoch nicht vermessen werden.

Bei der mittleren Abweichung zeigte sich weder bei der Thrombuslastmessung noch bei der Bestimmung der RV/LV-Ratio eine systematische Abweichung eines Untersucherpaares. Es gab keine starken Ausreißer bei der RV/LV-Ratio-Bestimmung; abweichende Untersuchungsergebnisse $>100 \%$ lagen bei der Thrombuslast stets bei $\leq 5,0 \%$. 
Tab. 8: Abweichungen der Untersuchungsergebnisse aller drei Auswerter.

\begin{tabular}{|l|c|c|c|}
\hline \multicolumn{1}{|c|}{ Untersuchung } & U1 vs. U2 & U1 vs. U3 & U2 vs. U3 \\
\hline mittlere Abweichung \\
\hline LPA-Obstruktion in \% & $16,1 \% \pm 31,3$ & $6,7 \% \pm 20,1$ & $23,6 \% \pm 109,7$ \\
RPA-Obstruktion in \% & $29,0 \% \pm 42,3$ & $12,8 \% \pm 51,5$ & $12,1 \% \pm 28,2$ \\
Thrombuslast gesamt * in \% & $26,2 \% \pm 36,4$ & $9,5 \% \pm 28,6$ & $12,5 \% \pm 22,0$ \\
RV/LV-Ratio & $3,7 \% \pm 4,7$ & $5,3 \% \pm 9,3$ & $9,1 \% \pm 8,3$ \\
\hline \multicolumn{4}{|l|}{ starke Ausreißer (>100\% Abweichung) } \\
LPA-Obstruktion in \% & $0(0,0 \%)$ & $3(1,5 \%)$ & $8(4,6 \%)$ \\
RPA-Obstruktion in \% & $0(0,0 \%)$ & $5(2,5 \%)$ & $3(1,7 \%)$ \\
Thrombuslast gesamt * in \% & $0(0,0 \%)$ & $3(1,5 \%)$ & $1(0,6 \%)$ \\
RV/LV-Ratio & $0(0,0 \%)$ & $0(0,0 \%)$ & $0(0,0 \%)$ \\
\hline
\end{tabular}

Angabe der prozentualen Abweichungen als Mittelwert mit zugehöriger Standardabweichung; Angabe der starken Ausreißer als Anzahl n der Patienten des jeweiligen Untersuchungskollektivs U1 vs. U3 n=65; U1 vs. U2 n=204; U2 vs. U3 n=173 mit dazugehörigem Prozentwert in Klammern. * definiert als Mittelwert des prozentualen Verschlusses bei Vorliegen eines Thrombus in LPA + RPA.

Um den Zusammenhang der Messergebnisse jeweils zweier Untersucher darzustellen, wurde für die kontinuierlichen Variablen der ICC bestimmt. Dieser Korrelationskoeffizient erlaubt nur eine Aussage über die Gleichförmigkeit der Untersuchungsergebnisse, aber keine über ihre tatsächliche Übereinstimmung. Es zeigten sich keine signifikanten Unterschiede zwischen den Ergebnissen der Untersucherpaare $(\mathrm{p}<0,001)$, der ICC war stets $>0,70$ (Tab. 9). Graphisch werden die Korrelationen in Abb. 8 und Abb. 9 dargestellt.

Für die nominalen Variablen wurde der Kappa-Koeffizient ermittelt. Dieser ist ein Maß für die tatsächliche Übereinstimmung der Auswertungsergebnisse zweier Untersucher. Mit Ausnahme eines Ausreißers lag $\kappa$ bei $>0,81$ und zeigte damit eine fast vollkommene Übereinstimmung der Messergebnisse aller Untersucherpaare $(\mathrm{p} \leq 0,001)$ (Tab. 9).

Tab. 9: Koeffizienten der Thrombuslast und RV/LV-Ratio aller drei Untersucher.

\begin{tabular}{|l|c|c|c|c|}
\hline \multicolumn{1}{|c|}{ Koeffizient } & U1 vs. U2 & U1 vs. U3 & U2 vs. U3 & p-Wert \\
\hline ICC & 0,702 & 0,988 & 0,967 & $<\mathbf{0 , 0 0 1}$ \\
\hline Thrombusgröße/Thrombuslast in \% & 0,987 & 0,983 & 0,947 & $<\mathbf{0 , 0 0 1}$ \\
RV/LV-Ratio & 0,329 & 0,933 & 0,915 & $\leq \mathbf{0 , 0 0 1}$ \\
\hline $\boldsymbol{\kappa}$ & 1,000 & 0,880 & 0,838 & $<\mathbf{0 , 0 0 1}$ \\
\hline mittlere Thrombuslast >50\% & 0,904 & 0,886 & 0,805 & $<\mathbf{0 , 0 0 1}$ \\
RV/LV-Ratio >1,0
\end{tabular}



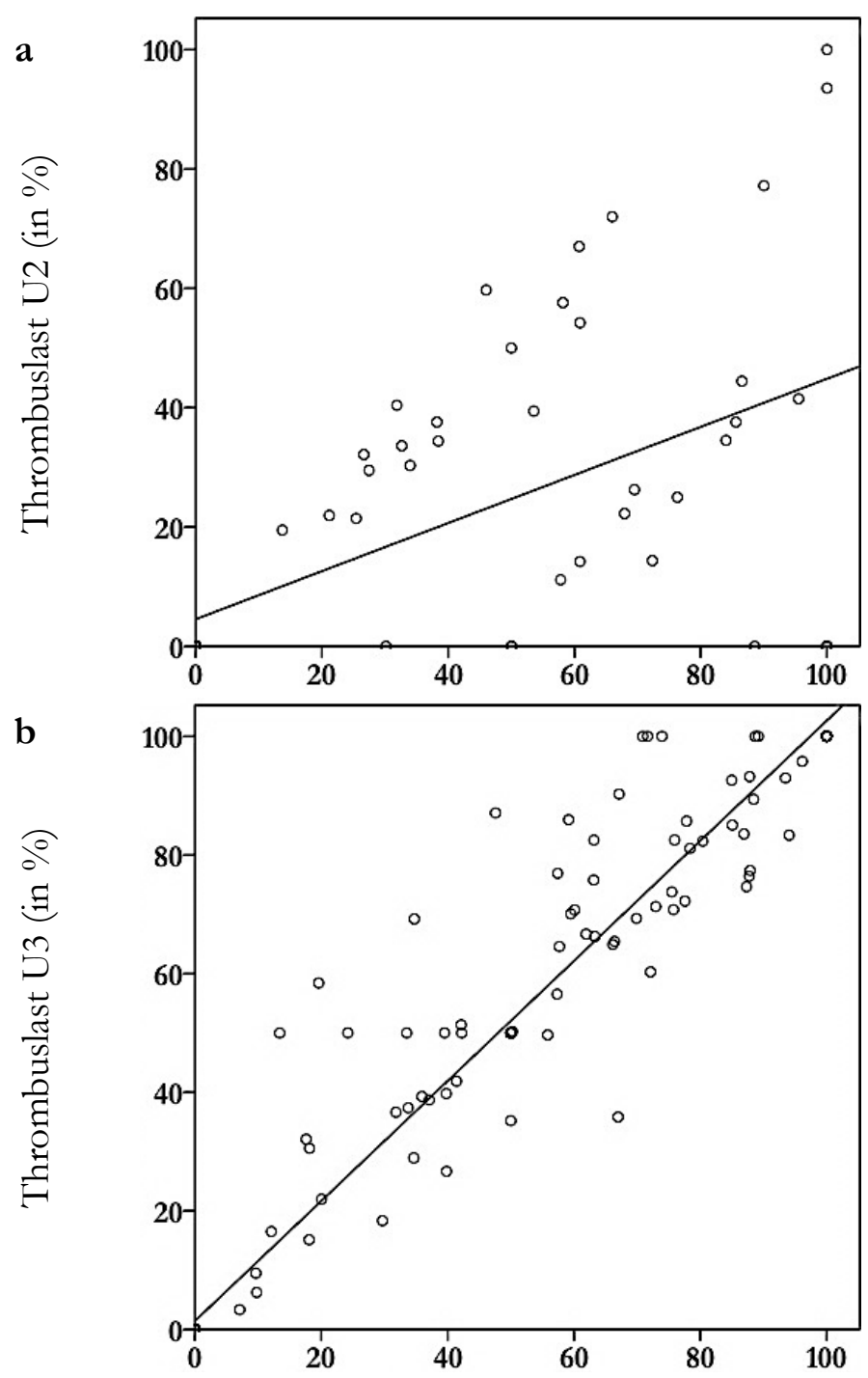

$\mathrm{R}^{2}$ Linear $=0,331$

$\mathrm{R}^{2}$ Linear $=0,956$

c

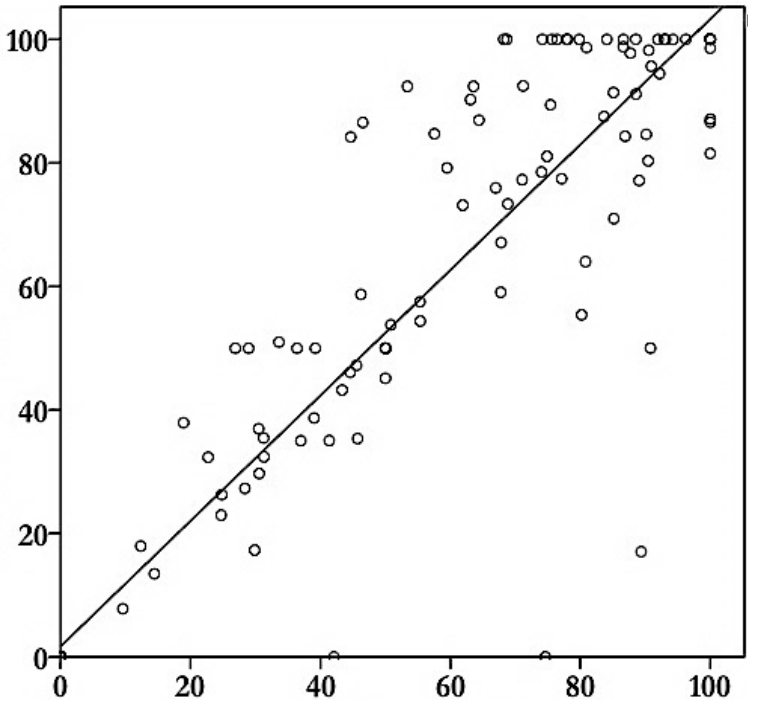

$\mathrm{R}^{2}$ Linear $=0,883$

Thrombuslast U1 (a+b) oder U2 (c) (in \%)

Abb. 8: Korrelation der prozentualen Thrombuslast-Messungen U1 vs. U2 (a), U1 vs. U3 (b) und U2 vs. U3 (c). 


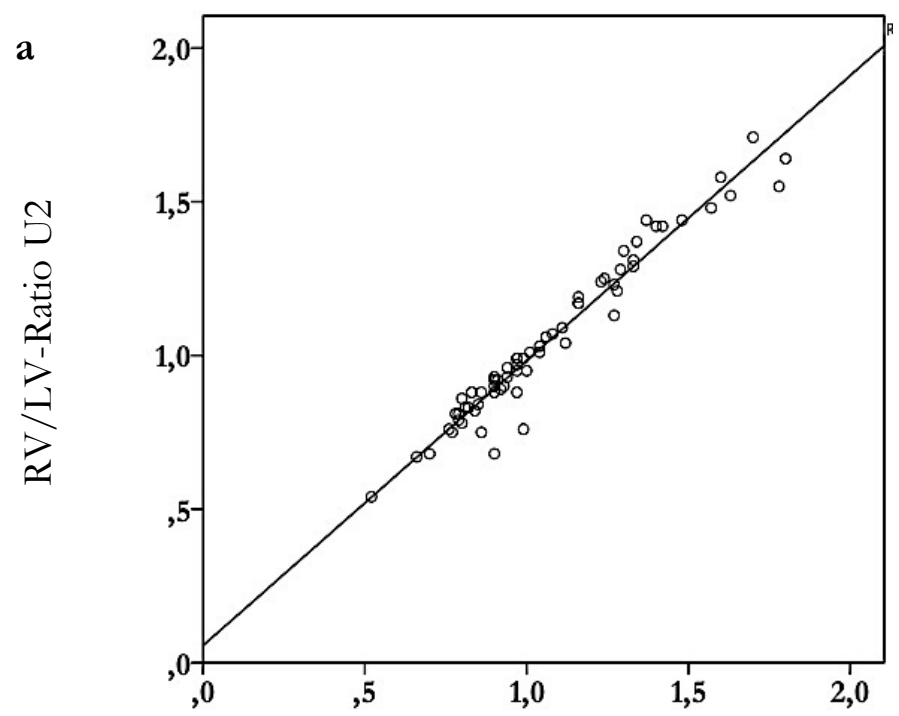

$\mathrm{R}^{2}$ Linear $=0,951$

b

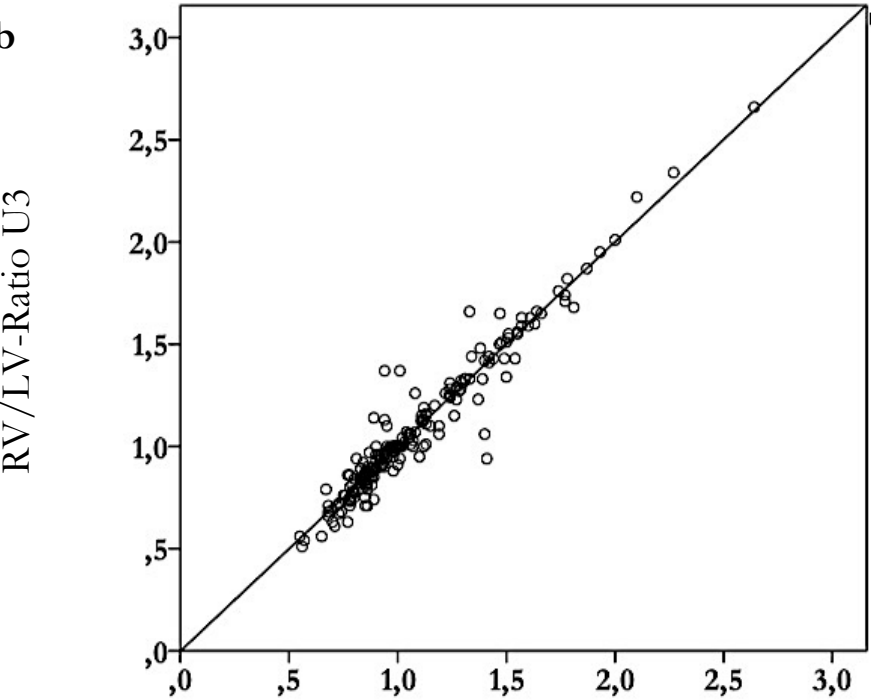

$\mathrm{R}^{2}$ Linear $=0,937$

c

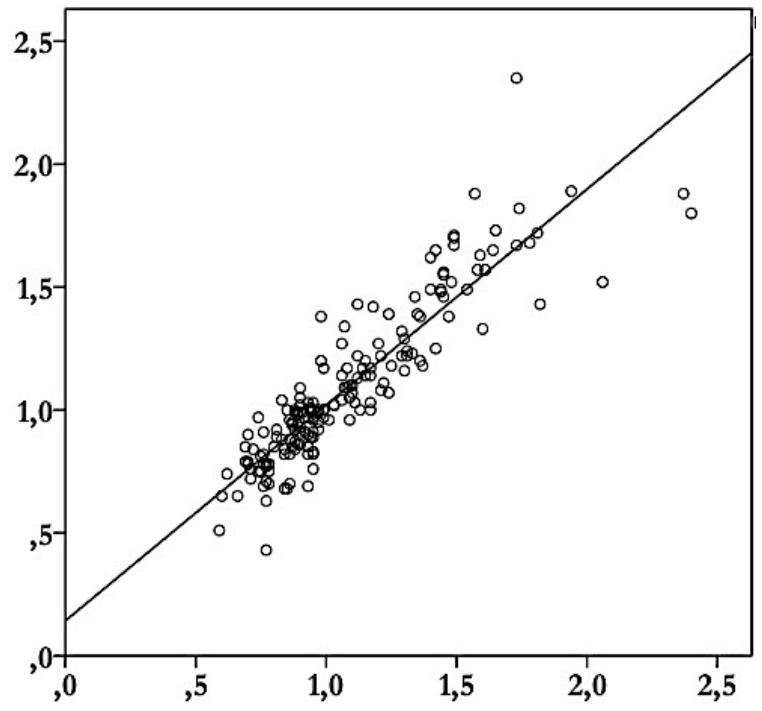

$\mathrm{R}^{2}$ Linear $=0,810$

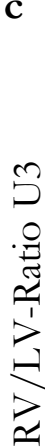

$\mathrm{RV} / \mathrm{LV}$-Ratio U1 (a+b) oder U2 (c)

Abb. 9: Korrelation der RV/LV-Ratio U1 vs. U2 (a), U1 vs. U3 (b) und U2 vs. U3 (c). 
Anhand des Vergleiches der mittleren Abweichungen und starken Ausreißer sowie der Überprüfung der Korrelation der Ergebnisse aller drei Untersucher miteinander ließ sich feststellen, dass die Interobserver-Variabilität gering war. Zwar ergaben sich Unterschiede in den Messungen und ihrer Gleichförmigkeit, doch waren diese nicht auf ein Untersucherpaar beschränkt, sondern variierten zwischen den verschiedenen UntersucherKombinationen. Daraus resultierend konnten die Ergebnisse aller drei Untersucher im Nachfolgenden zu Messwerterhebung 1 (M1) und Messwerterhebung 2 (M2) zusammengefasst werden. Die Zuordnung zu M1 oder M2 fand hierbei nach Aufnahmezeitpunkt des Patienten statt:

\section{M1 = Messungen von U1 oder U2, M2 = Messungen von U2 oder U3}

\subsubsection{Interobserver-Variabilität Messwerterhebung 1 vs. 2}

Für den graphischen Vergleich beider Untersucher und ihrer Messmethoden wurde ein Bland-Altman-Diagramm erstellt: hier ließen sich die Streuungen der einzelnen Differenzen darstellen sowie systematische Fehler, Ausreißer und individuelle Abweichungen hinsichtlich der Größenordnung und des Musters beurteilen. Bei der Messung der Thrombuslast lag der Mittelwert der Messdifferenzen bei $-2,5$ und die Standardabweichung bei $\pm 41,9$. Das 95\%-KI (Mittelwert $\pm 1,96$ x Standardabweichung) reichte von $-84,6$ bis 79,6, doch gab es auch einige Ausreißer ober- und vor allem unterhalb. Die mittleren Werte von M1 und M2 erstreckten sich um den Mittelwert der Differenzen herum über das gesamte Spektrum von nahezu 0\% bis 100\% (Abb. 10, Tab. 10). 


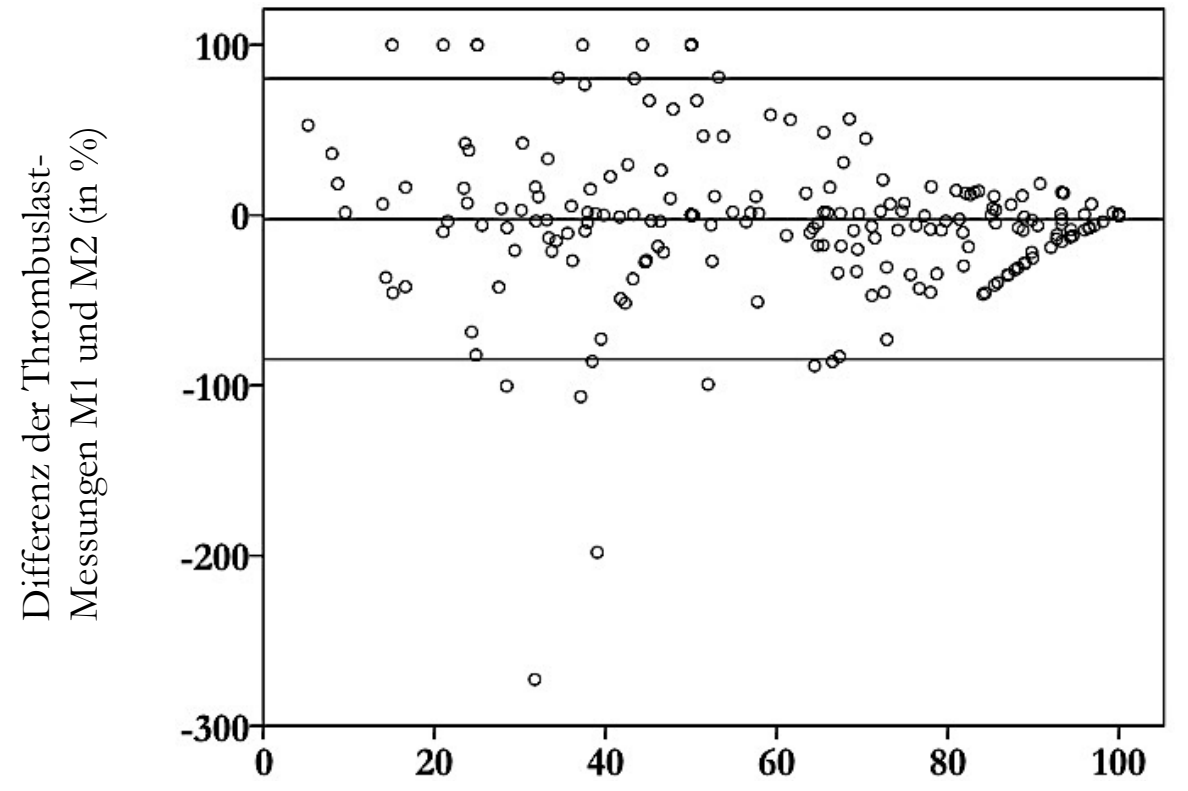

Mittelwert der Thrombuslast-Messungen M1 und M2 (in \%)

Abb. 10: Bland-Altman-Diagramm der Thrombuslast-Messungen von M1 und M2.

Tab. 10: Bland-Altman-Analyse der Thrombuslast-Messungen von M1 und M2.

\begin{tabular}{|l|c|}
\hline \multicolumn{1}{|c|}{ Variable } & Wert \\
\hline Mittelwert & $-2,5$ \\
Standardabweichung & $\pm 41,9$ \\
1,96 x Standardabweichung & 82,1 \\
Mittelwert + (1,96 x Standardabweichung) & 79,6 \\
Mittelwert - (1,96 x Standardabweichung) & $-84,6$ \\
\hline
\end{tabular}

Die Bland-Altman-Analyse der RV/LV-Ratio ergab einen Mittelwert der Messdifferenzen von $-0,5$ und eine Standardabweichung von $\pm 9,7$. Hier umfasste das 95\%-KI Werte von 19,5 bis 18,5. Die Mittelwerte von M1 und M2 konzentrierten sich vor allem auf Werte zwischen 0,5 und 1,5 um die mittlere Messdifferenz herum (Abb. 11, Tab. 11). 


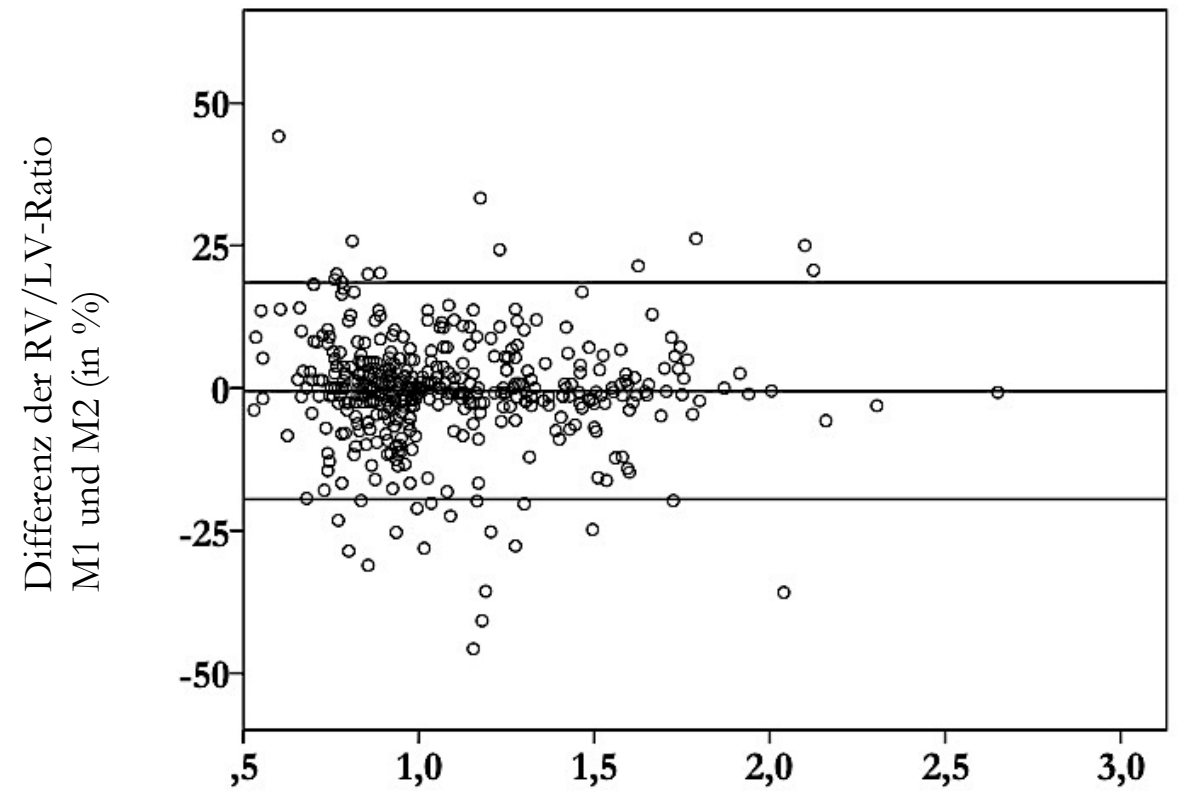

Mittelwert der RV/LV-Ratio M1 und M2

Abb. 11: Bland-Altman-Diagramm der RV/LV-Ratio von M1 und M2.

Tab. 11: Bland-Altman-Analyse der RV/LV-Ratio von M1 und M2.

\begin{tabular}{|l|c|}
\hline \multicolumn{1}{|c|}{ Variable } & Wert \\
\hline Mittelwert & $-0,5$ \\
Standardabweichung & $\pm 9,7$ \\
1,96 x Standardabweichung & 19,0 \\
Mittelwert + (1,96 x Standardabweichung) & 18,5 \\
Mittelwert - (1,96 x Standardabweichung) & $-19,5$ \\
\hline
\end{tabular}

\subsubsection{Intraobserver-Variabilität}

Nachdem die Unabhängigkeit der Messwertergebnisse von verschiedenen Untersuchern nachgewiesen wurde, sollte die Intraobserver-Variabilität, das heißt die Zuverlässigkeit und formale Genauigkeit der Messungen eines Untersuchers, bestimmt werden. Hierfür fand nach einem Jahr eine erneute Beurteilung von 50 CT-Bildern durch U1 statt, ohne Kenntnis der eigenen Erstbewertung (Tab. 12). Insgesamt blieben alle mittleren Abweichungen $<5 \%$. Der ICC konnte über alle untersuchten Parameter hinweg auf $>0,98$ festgelegt werden. Die Signifikanzprüfung ergab stets $\mathrm{p}<0,001$. 
Tab. 12: Abweichungen und Korrelation der Untersuchungsergebnisse von U1 zu zwei verschiedenen Zeitpunkten.

\begin{tabular}{|l|c|c|c|}
\hline \multicolumn{1}{|c|}{ Untersuchung } & mittlere Abweichung & ICC & p-Wert \\
\hline LPA-Obstruktion in \% & $2,68 \% \pm 7,35$ & 0,997 & $<\mathbf{0 , 0 0 1}$ \\
RPA-Obstruktion in \% & $2,38 \% \pm 7,18$ & 0,998 & $<\mathbf{0 , 0 0 1}$ \\
Thrombuslast gesamt* in \% & $3,61 \% \pm 7,80$ & 0,998 & $<\mathbf{0 , 0 0 1}$ \\
RV/LV-Ratio & $4,19 \% \pm 4,34$ & 0,987 & $<\mathbf{0 , 0 0 1}$ \\
\hline
\end{tabular}

Angabe der prozentualen Abweichungen als Mittelwert mit zugehöriger Standardabweichung.

* definiert als Mittelwert des prozentualen Verschlusses bei Vorliegen eines Thrombus in LPA + RPA.

\subsection{Erhobene computertomographische Befunde in der Studien- population}

\subsubsection{Thrombuslokalisation}

Die Auswertung der 442 MDCT-Bilder wurde nur hinsichtlich zentraler Thrombuslokalisationen durchgeführt: bei 4,8\% der Patienten fand sich ein Thrombus ausschließlich in der LPA, bei $12,2 \%$ in der RPA und 26,7\% litten an einem beidseitigen Gefäßverschluss. Ein alleiniger Thrombus im HS kam nicht vor; dort lokalisierte Thromben waren stets auf der Bifurkation reitend und wurden damit als in die RPA und LPA reichend gewertet. Diese in allen drei zentralen Gefäßen befindlichen Verschlüsse lagen bei 10,9\% der Patienten vor. In 45,5\% der Fälle konnte kein zentraler Thrombus nachgewiesen werden (Tab. 13).

Tab. 13: Häufigkeiten der verschiedenen Thrombuslokalisationen.

\begin{tabular}{|l|c|}
\hline \multicolumn{1}{|c|}{ Lokalisation } & Häufigkeit \\
\hline kein zentraler Thrombus & $201(45,5 \%)$ \\
Thrombus nur in LPA & $21(4,8 \%)$ \\
Thrombus nur in RPA & $54(12,2 \%)$ \\
Thrombus in LPA und RPA & $118(26,7 \%)$ \\
Thrombus nur im HS & $0(0,0 \%)$ \\
Thrombus in LPA, RPA und HS & $48(10,9 \%)$ \\
\hline
\end{tabular}

Angabe der Häufigkeit als Anzahl n der Patienten des Gesamtkollektivs n=442 mit dem korrespondierenden Prozentwert in Klammern. 


\subsubsection{Thrombusgröße}

Die im zweidimensionalen CT-Bild gemessene prozentuale Gefäßverlegung lag über die RPA und die LPA gemittelt bei 35,3\% mit einem etwas höheren Mittelwert in der RPA $(39,3 \%)$ als in der LPA $(31,4 \%)$. Auch beim Vorkommen einer Thrombuslast von $>50 \%$, $>90 \%$ und $=100 \%$ war die RPA gegenüber der LPA häufiger betroffen: $40,3 \%$ vs. $34,4 \%$, $22,6 \%$ vs. $14,9 \%$ und $19,5 \%$ vs. $12,2 \%$. Eine Verlegung von $>50 \%$ in LPA + RPA hatten 27,4\% der Patienten, eine beidseitige Thrombuslast von $>90 \%$ wurde bei $9,3 \%$ gefunden. Eine Okklusion von 100\% in LPA + RPA wurde bei 7,0\% des Patientenkollektivs festgestellt (Tab. 14).

Tab. 14: Prozentuale Obstruktion der Pulmonalgefäße und Häufigkeiten ausgewählter Prozentränge.

\begin{tabular}{|l|c|}
\hline \multicolumn{1}{|c|}{ Lokalisation } & prozentualer Verschluss \\
\hline LPA & $31,4 \% \pm 39,7$ \\
RPA & $39,3 \% \pm 42,4$ \\
Thrombuslast gesamt* & $35,3 \% \pm 37,5$ \\
\hline & Häufigkeit \\
\hline LPA $>50 \%$ Obstruktion & $152(34,4 \%)$ \\
RPA $>50 \%$ Obstruktion & $178(40,3 \%)$ \\
Thrombuslast LPA $>50 \%+$ RPA $>50 \%$ & $121(27,4 \%)$ \\
LPA $>90 \%$ Obstruktion & $66(14,9 \%)$ \\
RPA $>90 \%$ Obstruktion & $100(22,6 \%)$ \\
Thrombuslast LPA $>90 \%+$ RPA $>90 \%$ & $41(9,3 \%)$ \\
LPA $=100 \%$ Obstruktion & $54(12,2 \%)$ \\
RPA $=100 \%$ Obstruktion & $86(19,5 \%)$ \\
Thrombuslast LPA $=100 \%+$ RPA $=100 \%$ & $31(7,0 \%)$ \\
\hline
\end{tabular}

Angabe des prozentualen Verschlusses als Mittelwert mit zugehöriger Standardabweichung; Angabe der Häufigkeit als Anzahl n der Patienten des Gesamtkollektivs n=442 mit dem korrespondierenden Prozentwert in Klammern.

* definiert als Mittelwert des prozentualen Verschlusses bei Vorliegen eines Thrombus in LPA + RPA.

\subsubsection{Rechtsventrikulärer Diameter und RV/LV-Ratio}

Die Vermessung der Ventrikel ergab einen medianen RV-Wert von 44,2 $\mathrm{mm}$ und eine mediane RV/LV-Ratio von 0,99, was bedeutete, dass RV und LV nahezu gleich groß waren. Nachfolgende beispielhafte Untersuchungen verschiedener Cut-offs ergaben, dass 46,4\% des Patientenkollektivs eine RV/LV-Ratio $>1$ hatten und 36,7\% eine Ratio $>1,1$. Einen noch weiter vergrößerten RV und damit eine Ratio von $>1,3$ hatten 20,8\%, den Cut-off von $\geq 1,4$ erreichten $17,9 \%$ der Patienten (Tab. 15). 
Tab. 15: Medianer RV-Diameter und RV/LV-Ratio mit beispielhaften Cut-off-Werten.

\begin{tabular}{|l|c|}
\hline \multicolumn{1}{|c|}{ Rechter Ventrikel } & Median \\
\hline RV Diameter in $\mathrm{mm}$ & $44,2[38,4-50,1]$ \\
RV/LV-Ratio & $0,99[0,86-1,26]$ \\
\hline & Häufigkeit \\
\hline RV/LV-Ratio $>1$ & $205(46,4 \%)$ \\
RV/LV-Ratio $>1,1$ & $162(36,7 \%)$ \\
RV/LV-Ratio $>1,3$ & $92(20,8 \%)$ \\
RV/LV-Ratio $\geq 1,4$ & $79(17,9 \%)$ \\
\hline
\end{tabular}

Angabe der kontinuierlichen Variablen als Median mit dazugehörigem IQR in eckigen Klammern; Angabe der Häufigkeit als Anzahl n der Patienten des Gesamtkollektivs n=442 mit dem korrespondierenden Prozentwert in Klammern.

\subsection{Risikostratifizierung mittels Multidetektor-Computertomographie}

\subsubsection{Prädiktiver Wert der computertomographischen Parameter}

Innerhalb des Studienkollektivs blieben 384 (86,9\%) Patienten hämodynamisch stabil, 58 $(13,1 \%)$ hingegen nicht. Die Betrachtung der Lokalisation der zentralen Thromben ergab, dass nur das Vorliegen eines auf der Bifurkation des HS reitenden Thrombus signifikant mit dem Erreichen des primären EP assoziiert war (19,0\% vs. 9,6\%, p=0,042). Gefäßverschlüsse in der LPA, RPA oder in LPA + RPA blieben ohne Einfluss. Ebenfalls ohne Auswirkungen für eine hämodynamische Instabilität war die Thrombusgröße. Weder war der prozentuale Gefäßverschluss signifikant höher ( $p=0,333$ bzw. $p=0,790$ bzw. $p=0,607$ ) noch sorgte eine Thrombuslast $>50 \%,>90 \%$ oder $=100 \%$ für das signifikant häufigere Erreichen des primären EP ( $p=0,140$ bis $p=1,000)$. Der Durchmesser des RV war in der Patientengruppe, die den primären EP erreichte, nicht signifikant größer (45,3 mm vs. 43,8 $\mathrm{mm}, \mathrm{p}=0,054)$. Jedoch war die RV/LV-Ratio mit 1,15 vs. 0,98 signifikant vergrößert $(p=0,002)$ bei Patienten am primären EP und die Cut-offs von RV/LV $>1$ bis $\geq 1,4$ waren häufiger anzutreffen: RV/LV >1: $60,3 \%$ vs. $44,3 \%$ ( $p=0,024),>1,1: 51,7 \%$ vs. $34,4 \%$ $(p=0,013),>1,3: 39,7 \%$ vs. $18,0 \%(p<0,001), \geq 1,4: 34,5 \%$ vs. $15,4 \%(p<0,001)$ (Tab. 16). 
Tab. 16: Prädiktiver Wert der CT-Parameter für das Erreichen des primären EP.

\begin{tabular}{|c|c|c|c|}
\hline Parameter & $\begin{array}{c}\text { EP nicht erreicht } \\
(n=384)\end{array}$ & $\begin{array}{c}\text { EP erreicht } \\
(n=58)\end{array}$ & p-Wert \\
\hline \multicolumn{4}{|l|}{ Thrombuslokalisation } \\
\hline Thrombus in LPA & $18(4,7 \%)$ & $3(5,2 \%)$ & 0,747 \\
\hline Thrombus in RPA & $50(13,0 \%)$ & $4(6,9 \%)$ & 0,280 \\
\hline Thrombus in LPA + RPA & $104(27,1 \%)$ & $14(24,1 \%)$ & 0,751 \\
\hline Thrombus in LPA + RPA + HS & $37(9,6 \%)$ & $11(19,0 \%)$ & 0,042 \\
\hline \multicolumn{4}{|l|}{ Thrombusgröße/Thrombuslast } \\
\hline LPA-Obstruktion in $\%$ & $0,0[0,0-68,5]$ & $0,0[0,0-79,0]$ & 0,333 \\
\hline RPA-Obstruktion in $\%$ & $22,0[0,0-85,2]$ & $6,4[0,0-87,4]$ & 0,790 \\
\hline Thrombuslast gesamt $*$ in $\%$ & $24,9[0,0-68,9]$ & $39,8[0,0-80,3]$ & 0,607 \\
\hline LPA $>50 \%$ Obstruktion & $127(33,1 \%)$ & $25(43,1 \%)$ & 0,140 \\
\hline $\mathrm{RPA}>50 \%$ Obstruktion & $153(39,8 \%)$ & $25(43,1 \%)$ & 0,668 \\
\hline Thrombuslast LPA $>50 \%+\mathrm{RPA}>50 \%$ & $102(26,6 \%)$ & $19(32,8 \%)$ & 0,344 \\
\hline LPA $>90 \%$ Obstruktion & $58(15,1 \%)$ & $8(13,8 \%)$ & 1,000 \\
\hline RPA $>90 \%$ Obstruktion & $86(22,4 \%)$ & $14(24,1 \%)$ & 0,739 \\
\hline Thrombuslast LPA $>90 \%+\mathrm{RPA}>90 \%$ & $36(9,4 \%)$ & $5(8,6 \%)$ & 1,000 \\
\hline $\mathrm{LPA}=100 \%$ Obstruktion & $48(12,5 \%)$ & $6(10,3 \%)$ & 0,830 \\
\hline $\mathrm{RPA}=100 \%$ Obstruktion & $74(19,3 \%)$ & $12(20,7 \%)$ & 0,859 \\
\hline Thrombuslast LPA $=100 \%+\mathrm{RPA}=100 \%$ & $28(7,3 \%)$ & $3(5,2 \%)$ & 0,783 \\
\hline \multicolumn{4}{|l|}{ Rechter Ventrikel } \\
\hline RV-Diameter in $\mathrm{mm}$ & $43,8[38,3-49,7]$ & $45,3[39,1-53,7]$ & 0,054 \\
\hline RV/LV-Ratio & $0,98[0,85-1,23]$ & $1,15[0,93-1,52]$ & 0,002 \\
\hline $\mathrm{RV} / \mathrm{LV}$-Ratio $>1,0$ & $170(44,3 \%)$ & $35(60,3 \%)$ & 0,024 \\
\hline $\mathrm{RV} / \mathrm{LV}$-Ratio $>1,1$ & $132(34,4 \%)$ & $30(51,7 \%)$ & 0,013 \\
\hline $\mathrm{RV} / \mathrm{LV}$-Ratio $>1,3$ & $69(18,0 \%)$ & $23(39,7 \%)$ & $<0,001$ \\
\hline $\mathrm{RV} / \mathrm{LV}$-Ratio $\geq 1,4$ & $59(15,4 \%)$ & $20(34,5 \%)$ & $<0,001$ \\
\hline
\end{tabular}

Angabe der kategorialen Variablen als Anzahl $n$ der Patienten des jeweiligen Patientenkollektivs $\mathrm{n}=384$ bzw. $\mathrm{n}=58$ mit dem korrespondierenden Prozentwert in Klammern; Angabe der kontinuierlichen Variablen als Median mit dazugehörigem IQR in eckigen Klammern.

* definiert als Mittelwert des prozentualen Verschlusses bei Vorliegen eines Thrombus in LPA + RPA.

\subsubsection{Weiterführende Untersuchung der signifikanten Parameter}

Nachdem festgestellt werden konnte, dass nur ein im HS reitender Thrombus, d. h. ein Thrombus im HS + LPA + RPA, und eine RV/LV-Ratio von mindestens $>1$ signifikant mit dem Erreichen des primären EP vergesellschaftet waren, sollten diese nachfolgend näher untersucht werden.

Bei genauerer Betrachtung hatten Patienten mit einem Thrombus im HS + LPA + RPA ein 2,2-fach $[1,0-4,6](p=0,037)$ höheres Risiko für hämodynamisch relevante Komplikationen. Bezogen auf die Mortalität jeglicher Ursache $(\mathrm{p}=0,483)$ oder auf die alleinige 
Betrachtung des Todes als Folge der akuten LE $(p=0,403)$ gab es keinen signifikanten Zusammenhang.

Zur Feststellung des Einflusses der RV/LV-Ratio wurde zunächst der optimale Cutoff-Wert berechnet. Die ROC-Analyse ergab eine AUC von 0,628 [0,544-0,712] $(\mathrm{p}=0,002)$, wobei zu bemerken ist, dass im Bereich einer Sensitivität von 0,8-1,0 bzw. einer Spezifität von 0,0-0,2 die Ratewahrscheinlichkeit (AUC=0,5) unterschritten wurde (Abb. 12). Nach Anwendung des Youden-Index wurde eine RV/LV-Ratio >1,3 als optimaler Cut-off-Wert identifiziert. Die Prüfung seiner prognostischen Performance ergab eine Sensitivität von 0,40 [0,28-0,53], eine Spezifität von 0,82 [0,78-0,86], einen PPW von 0,25 [0,17-0,35] und einen NPW von 0,90 [0,86-0,93]. Es konnte eine OR von 3,0 [1,7-5,4] (p<0,001) für das Erreichen des primären EP bei einer RV/LV-Ratio >1,3 und eine OR von 3,3 [1,3-8,3] $(p=0,01)$ für eine Todesfolge aufgrund der akuten LE festgestellt werden. Eine Assoziation dieses Cut-offs mit dem Exitus letalis in Folge jeglicher Ursache gab es nicht (OR 1,5, $\mathrm{p}=0,335)$.

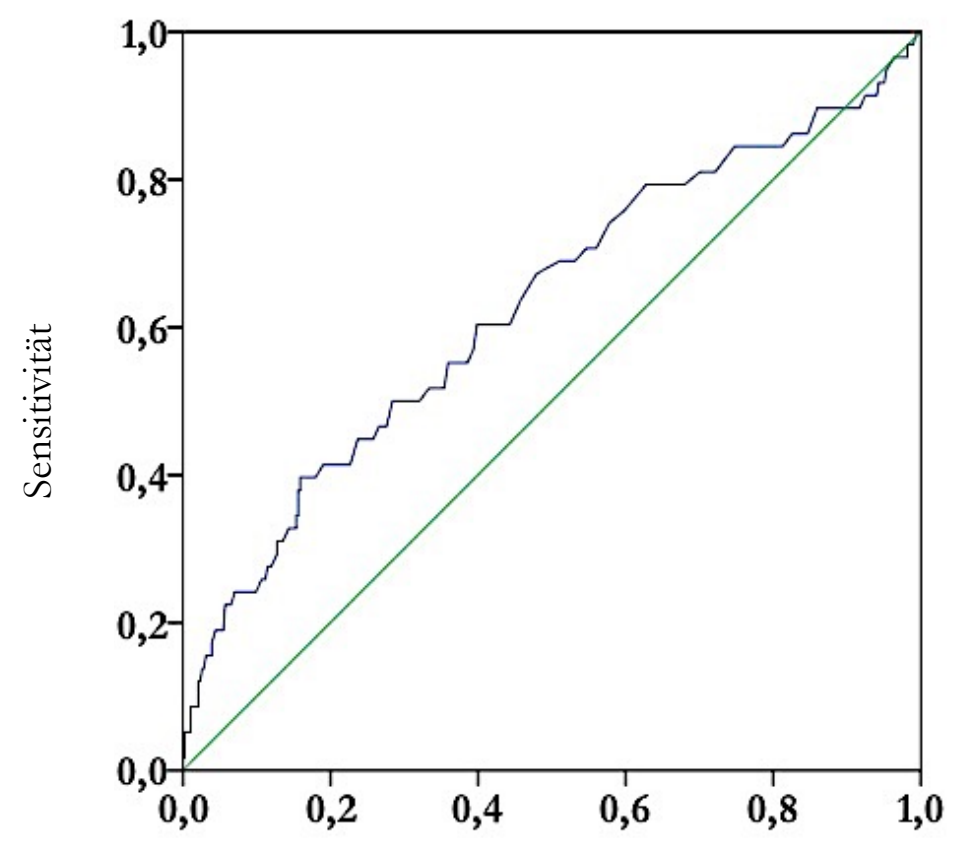

1-Spezifität

Abb. 12: ROC-Kurve der RV/LV-Ratio im Hinblick auf das Erreichen des primären EP.

Die Thrombuslokalisation im HS + LPA + RPA bzw. die RV/LV >1,3 besaßen eine Spezifität von $0,90[0,87-0,93]$ bzw. $0,82[0,78-0,86]$. Im Vergleich mit etablierten klinischen 
und laborchemischen Parametern zeigten sich bei Vorliegen einer Hypotension $(0,94$ [0,91$0,96])$, einer Hypoxie $(0,75$ [0,70-0,79]) oder einer Synkope $(0,90[0,87-0,93])$ ähnliche Werte. Aufgrund der schwachen Sensitivität der Thrombuslokalisation $(0,19$ [0,11-0,31]) und der RV/LV-Ratio >1,3 (0,40 [0,28-0,53]) eigneten sich diese im Gegensatz zu einem sPESI $\geq 1 \quad(0,95[0,86-0,98])$, hsTnT $\geq 14 \mathrm{pg} / \mathrm{ml}(0,95[0,84-0,99])$ und NT-proBNP $\geq 600 \mathrm{pg} / \mathrm{ml}(0,79[0,64-0,88])$ jedoch nicht zur eindeutigen Identifikation von Patienten mit einem gesteigerten Risiko für hämodynamisch relevante Komplikationen. Die OR war bei den Ergebnissen der vorliegenden Arbeit zwar mit 2,2 [1,0-4,6] $(p=0,037)$ bzw. 3,0 $[1,7-5,4](p<0,001)$ leicht erhöht, doch lag diese bei den klinischen und laborchemischen Parametern mit Werten bis zu OR=16,6 [8,1-34,1] $(\mathrm{p}<0,001)$ deutlich höher (Tab. 17).

Tab. 17: Prognostische Performances der Thrombuslokalisation im HS + LPA + RPA und der RV/LV-Ratio >1,3 vs. ausgewählte klinische und laborchemische Parameter.

\begin{tabular}{|l|c|c|c|c|}
\hline \multicolumn{1}{|c|}{ Parameter } & Sensitivität & Spezifität & OR & p-Wert \\
\hline Studienergebnisse & $0,19[0,11-0,31]$ & $0,90[0,87-0,93]$ & $2,2[1,0-4,6]$ & $\mathbf{0 , 0 3 7}$ \\
\hline Thrombuslokalisation im HS & $0,40[0,28-0,53]$ & $0,82[0,78-0,86]$ & $3,0[1,7-5,4]$ & $<\mathbf{0 , 0 0 1}$ \\
RV/LV-Ratio $>1,3$ & |l|l|l| \\
\hline \multicolumn{4}{|l|}{ klinische und laborchemische Parameter } \\
\hline Hypotension & $0,52[0,38-0,66]$ & $0,94[0,91-0,96]$ & $16,6[8,1-34,1]$ & $<\mathbf{0 , 0 0 1}$ \\
Hypoxie & $0,62[0,49-0,74]$ & $0,75[0,70-0,79]$ & $5,9[2,7-9,1]$ & $<\mathbf{0 , 0 0 1}$ \\
Synkope & $0,39[0,27-0,52]$ & $0,90[0,87-0,93]$ & $5,7[3,0-10,7]$ & $<\mathbf{0 , 0 0 1}$ \\
sPESI $\geq 1$ & $0,95[0,86-0,98]$ & $0,35[0,31-0,40]$ & $9,9[3,1-32,3]$ & $<\mathbf{0 , 0 0 1}$ \\
hsTnT $\geq 14 \mathrm{pg} / \mathrm{ml}$ & $0,95[0,84-0,99]$ & $0,38[0,33-0,44]$ & $12,2[2,9-51,4]$ & $\mathbf{0 , 0 0 1}$ \\
NT-proBNP $\geq 600 \mathrm{pg} / \mathrm{ml}$ & $0,79[0,64-0,88]$ & $0,48[0,43-0,53]$ & $3,4[1,6-7,3]$ & $\mathbf{0 , 0 0 2}$ \\
\hline
\end{tabular}

Angabe der Sensitivität und Spezifität mit zugehörigem 95\%-KI in eckigen Klammern; Angabe der OR mit zugehörigem 95\%-KI in eckigen Klammern und Signifikanzprüfung.

\subsubsection{Kombination von Thrombuslast und RV/LV-Ratio}

Innerhalb des Studienkollektivs ( $\mathrm{n}=442)$ fanden sich bei 25 Patienten $(5,7 \%)$ gleichzeitig ein Thrombus im HS + LPA + RPA und eine RV/LV-Ratio >1,3. Es erreichten sieben Patienten dieser Gruppe (28,0\%) den primären EP, 18 Patienten (72,0\%) blieben ohne hämodynamisch relevante Komplikationen $(\mathrm{p}=0,033)$. Bei Vorliegen eines Thrombus im HS + LPA + RPA $(n=48,10,9 \%)$ war die mediane RV/LV-Ratio signifikant höher als beim Fehlen eines solchen: 1,34 [1,15-1,60] vs. $0,97[0,85-1,20](\mathrm{p}<0,001)$. In der zugehörigen ROC-Analyse konnte diese Assoziation graphisch dargestellt werden (AUC=0,771 
[0,703-0,840], $\mathrm{p}<0,001$ ) (Abb. 13). Die OR für die Entwicklung einer hämodynamischen Instabilität bei Vorliegen eines Thrombus im HS + LPA + RPA und einer RV/LV-Ratio $>1,3$ lag bei 1,8 $[0,5-7,4](p=0,386)$. Es bestand somit kein erhöhtes Risiko für das Erreichen des primären EP innerhalb dieser Patientengruppe. Die Untersuchung der prognostischen Performances der Kombination von Thrombuslokalisation im HS + LPA + RPA und RV/LV-Ratio >1,3 ergab eine niedrigere Sensitivität von 0,12 [0,06-0,23] im Vergleich zu derjenigen der Einzelkomponenten $(0,19$ bzw. 0,40) und eine höhere Spezifität von 0,95 [0,93-0,97] gegenüber 0,90 bzw. 0,82.

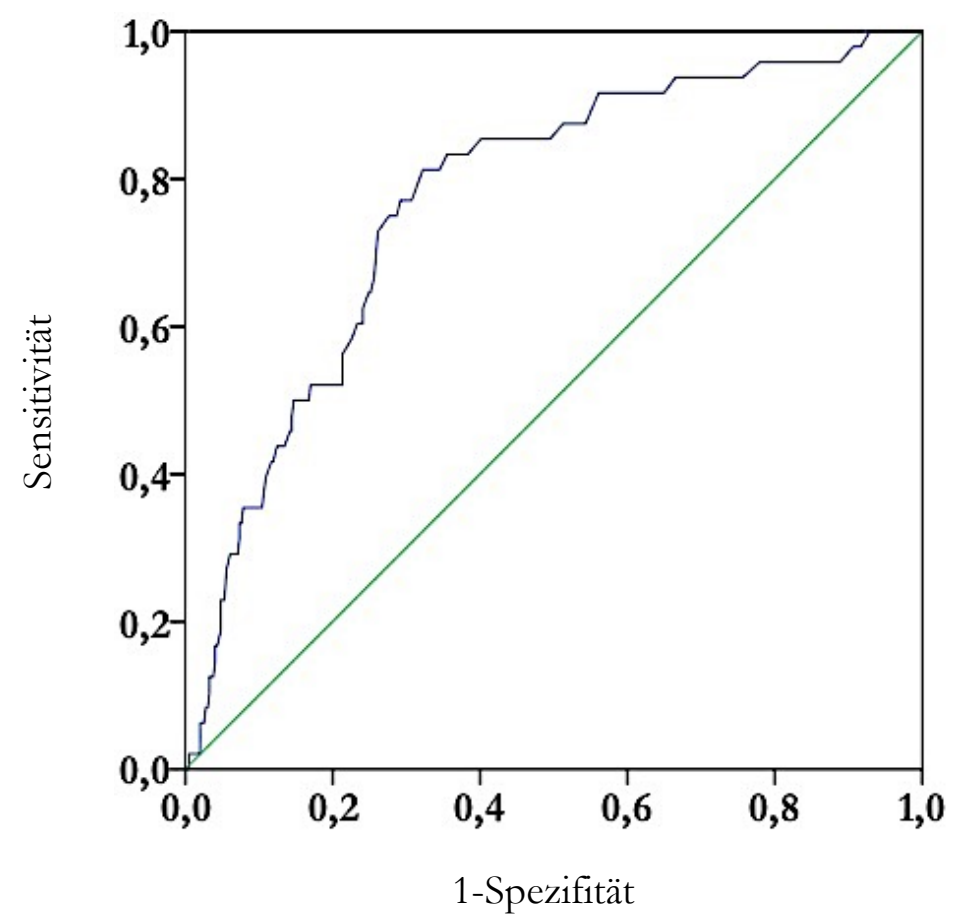

Abb. 13: ROC-Kurve der RV/LV-Ratio bei Vorliegen eines zentralen Thrombus.

In der Studienpopulation ( $\mathrm{n}=442$ ) lag eine Thrombuslast $>50 \%$ in LPA + RPA bei 121 Patienten vor (27,4\%). Hiervon erreichten 19 Patienten (15,7\%) den primären EP, bei 102 $(84,3 \%)$ war dies nicht der Fall $(p=0,344)$. Die Thrombusgröße allein blieb in den vorangegangenen Untersuchungen ohne signifikante Auswirkung auf das Auftreten von Komplikationen, weshalb im Folgenden der zusätzliche Einfluss der RV/LV-Ratio betrachtet werden sollte. Patienten mit einer beidseitigen Thrombuslast $>50 \%$ hatten eine signifikant größere mediane RV/LV-Ratio $(1,28$ [1,08-1,57]) als Patienten mit einem geringeren Gefäßverschluss $(0,93$ [0,83-1,09]) ( $\mathrm{p}<0,001)$. Graphisch dargestellt wird diese Assoziation in der ROC-Analyse (AUC=0,815 [0,771-0,859], $\mathrm{p}<0,001)$ (Abb. 14). 


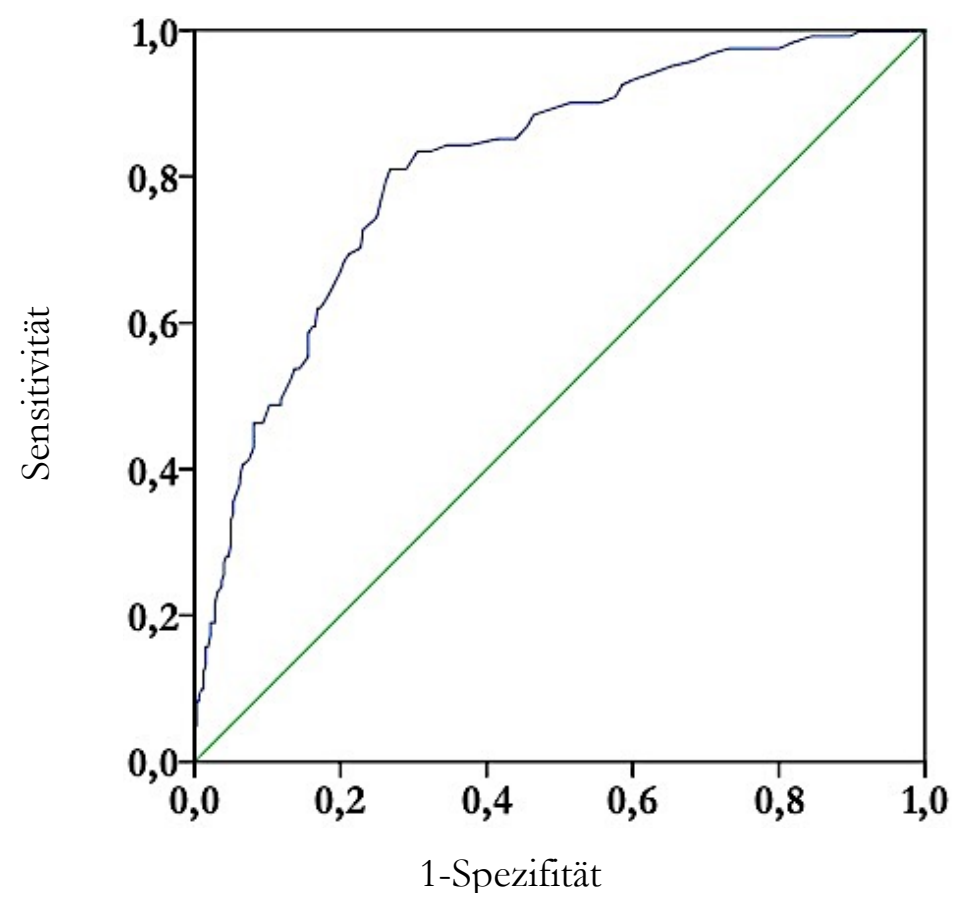

Abb. 14: ROC-Kurve der RV/LV-Ratio bei Vorliegen einer Thrombuslast in LPA $>50 \%$ $+\mathrm{RPA}>50 \%$.

Innerhalb der Gruppe mit einem bilateralen Gefäßverschluss $>50 \%$ war bei Erreichen des primären $\mathrm{EP}(\mathrm{n}=19,32,8 \%)$ die RV/LV-Ratio ebenfalls vergrößert: 1,50 [1,37-1,92] vs. $1,25[1,07-1,52](p=0,001)$. Die ROC-Analyse ergab hier eine AUC von 0,735 [0,622-0,847] $(\mathrm{p}=0,001)$ (Abb. 15). Mittels Youden-Index konnte für diese Patientengruppe ein optimaler Cut-off von RV/LV >1,3 für das Erreichen des primären EP berechnet werden, die zugehörige OR lag bei 4,9 [1,5-15,9] $(\mathrm{p}=0,007)$. Patienten mit einer Thrombuslast in LPA $>50 \%+\mathrm{RPA}>50 \%$ und einer RV/LV-Ratio $>1,3$ hatten somit ein 4,9-fach erhöhtes Risiko, den primären EP zu erreichen, wohingegen die Verlegung beider Gefäße $>50 \%$ allein keinen Einfluss auf einen komplizierten Krankheitsverlauf hatte.

Die Kombination der Thrombuslast mit einer RV/LV-Ratio $>1,3$ erbrachte bei Betrachtung der Thrombuslokalisation keine zusätzlichen Informationen bezüglich des Erreichens des primären EP, bei Fokus auf den prozentualen Gefäßverschluss dagegen schon. 


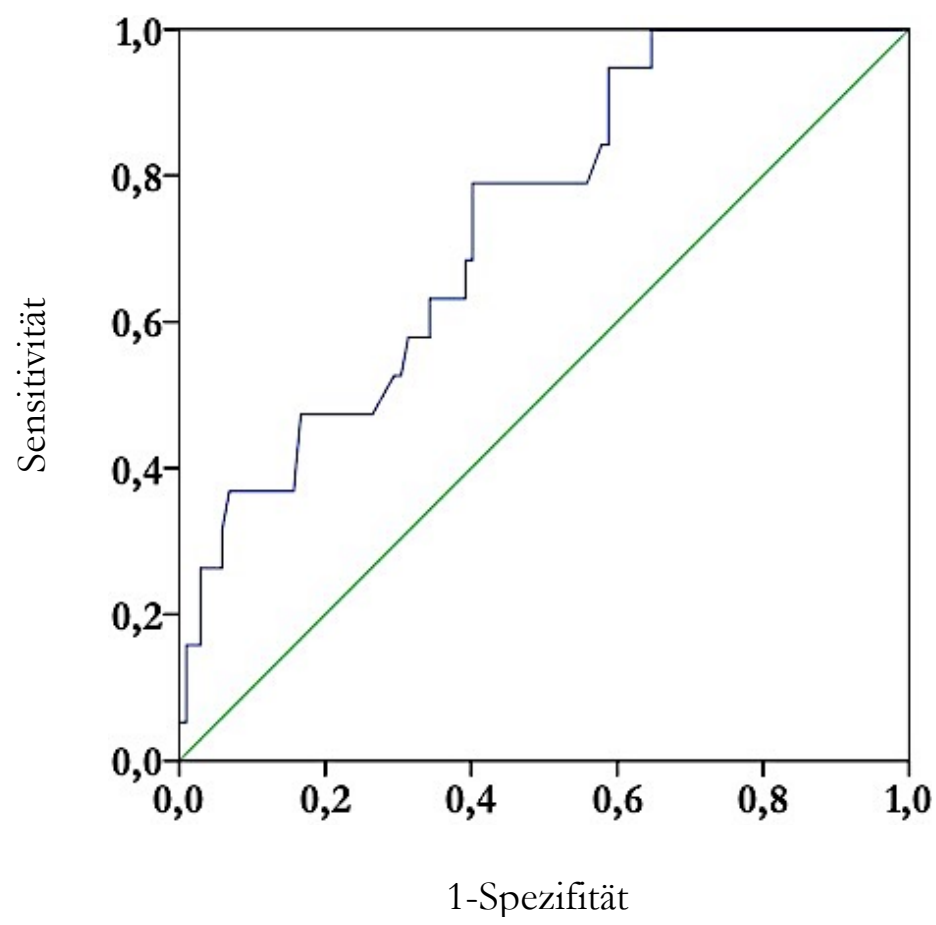

Abb. 15: ROC-Kurve der RV/LV-Ratio bei Vorliegen einer Thrombuslast in LPA $>50 \%$ $+\mathrm{RPA}>50 \%$ im Hinblick auf das Erreichen des primären EP.

\subsubsection{Subgruppenanalyse: Normotensive Patienten}

In der folgenden Subgruppenanalyse wurden alle Patienten mit einer initial durch den ESC2014-Score identifizierten Hochrisiko-LE ausgeschlossen ( $n=35,7,9 \%)$. Damit umfasste das Patientenkollektiv noch 407 Patienten, von denen 24 (5,9\%) den primären EP erreichten, 383 (94,1\%) dagegen nicht. Innerhalb Ersterer erlitten - bezogen auf die gesamte Subgruppe - 2,2\% einen Exitus letalis als Folge der akuten LE, die gesamte Mortalität lag bei 3,9\%. Ein nach ESC-2014-Score intermediär-hohes Risiko für Komplikationen hatten 128 (31,4\%) Patienten der Subgruppe, den primären EP erreichten 10 (41,7\%) dieser Patienten. Die Zahl der Patienten innerhalb der Subgruppe mit nach ESC-2014-Score intermediärniedrigem oder niedrigem Risiko für einen komplizierten Krankheitsverlauf betrug 279 (68,6\%). Eine hämodynamische Instabilität trat bei 14 (58,3\%) dieser Patienten auf.

In der Subgruppe der normotensiven Patienten war ein im HS + LPA + RPA gelegener Thrombus anders als in der gesamten Studienpopulation kein Prädiktor mehr für einen komplizierten Krankheitsverlauf. Bei der Thrombusgröße gab es hinsichtlich der prognostischen Aussagekraft keinen Unterschied zum Gesamtkollektiv: Gefäßverlegungen $>50 \%$, $>90 \%$ und $=100 \%$ blieben ohne Auswirkung auf das Erreichen des primären EP. Der Diameter des RV und die RV/LV-Ratio waren ebenfalls ohne Einfluss; der für die gesamte 
Studienpopulation bestimmte Cut-off-Wert von RV/LV >1,3 konnte nicht zwischen Patienten mit schlechtem Verlauf und solchen ohne unterscheiden $(25,0 \%$ vs. $18,0 \%$, $\mathrm{p}=0,415$ ) (Tab. 18). Bei Differenzierung innerhalb der Subgruppe normotensiver Patienten in Patienten mit nach ESC-2014-Score intermediär-hohem Risiko und intermediärniedrigem bzw. niedrigem Risiko für das Auftreten von Komplikationen ließ sich in der Patientengruppe mit intermediär-hohem Risiko kein prädiktiver Risikofaktor für das Erreichen des primären EP identifizieren (Tab. 19). Lag bei Patienten ein intermediär-niedriges oder niedriges Risiko für eine hämodynamische Instabilität vor, erreichten Patienten mit einem Thrombus im HS + LPA + RPA signifikant häufiger den primären EP als Patienten ohne einen reitenden Thrombus (21,4\% vs. 5,3\%, $\mathrm{p}=0,045)$ (Tab. 20). Alle übrigen untersuchten CT-Parameter blieben wie schon in der gesamten Subgruppe ohne Vorhersagekraft für das Auftreten von Komplikationen.

Tab. 18: Prädiktiver Wert der CT-Parameter für das Erreichen des primären EP.

\begin{tabular}{|c|c|c|c|}
\hline Parameter & $\begin{array}{c}\text { EP nicht erreicht } \\
(n=383)\end{array}$ & $\begin{array}{c}\text { EP erreicht } \\
(n=24)\end{array}$ & p-Wert \\
\hline \multicolumn{4}{|l|}{ Thrombuslokalisation } \\
\hline Thrombus in LPA & $18(4,7 \%)$ & $1(4,2 \%)$ & 1,000 \\
\hline Thrombus in RPA & $50(13,1 \%)$ & $2(8,3 \%)$ & 0,754 \\
\hline Thrombus in LPA + RPA & $104(27,2 \%)$ & $3(12,5 \%)$ & 0,152 \\
\hline Thrombus in LPA + RPA + HS & $37(9,7 \%)$ & $5(20,8 \%)$ & 0,088 \\
\hline \multicolumn{4}{|l|}{ Thrombusgröße/Thrombuslast } \\
\hline LPA-Obstruktion in $\%$ & $0,0[0,0-68,5]$ & $0,0[0,0-81,2]$ & 0,901 \\
\hline RPA-Obstruktion in $\%$ & $27,9[0,0-85,2]$ & $0,0[0,0-90,3]$ & 0,813 \\
\hline Thrombuslast gesamt $*$ in $\%$ & $25,0[0,0-69,0]$ & $0,0[0,0-83,7]$ & 0,810 \\
\hline LPA $>50 \%$ Obstruktion & $127(33,2 \%)$ & $9(37,5 \%)$ & 0,660 \\
\hline RPA $>50 \%$ Obstruktion & $153(39,9 \%)$ & $10(41,7 \%)$ & 1,000 \\
\hline Thrombuslast LPA $>50 \%+\mathrm{RPA}>50 \%$ & $102(26,6 \%)$ & $8(33,3 \%)$ & 0,481 \\
\hline LPA $>90 \%$ Obstruktion & $58(15,1 \%)$ & $3(12,5 \%)$ & 1,000 \\
\hline RPA $>90 \%$ Obstruktion & $86(22,5 \%)$ & $6(25,0 \%)$ & 0,802 \\
\hline Thrombuslast LPA $>90 \%+\mathrm{RPA}>90 \%$ & $36(9,4 \%)$ & $3(12,5 \%)$ & 0,492 \\
\hline $\mathrm{LPA}=100 \%$ Obstruktion & $48(12,5 \%)$ & $1(4,2 \%)$ & 0,337 \\
\hline $\mathrm{RPA}=100 \%$ Obstruktion & $74(19,3 \%)$ & $5(20,8 \%)$ & 0,794 \\
\hline Thrombuslast LPA $=100 \%+\mathrm{RPA}=100 \%$ & $28(7,3 \%)$ & $1(4,2 \%)$ & 1,000 \\
\hline \multicolumn{4}{|l|}{ Rechter Ventrikel } \\
\hline RV-Diameter in $\mathrm{mm}$ & $43,9[38,3-49,7]$ & $43,7[34,7-54,3]$ & 0,652 \\
\hline RV/LV-Ratio & $0,98[0,85-1,23]$ & $1,03[0,68-1,40]$ & 0,488 \\
\hline $\mathrm{RV} / \mathrm{LV}$-Ratio $>1,3$ & $69(18,0 \%)$ & $6(25,0 \%)$ & 0,415 \\
\hline
\end{tabular}

Angabe der kategorialen Variablen als Anzahl n der Patienten des jeweiligen Patientenkollektivs $\mathrm{n}=383$ bzw. $\mathrm{n}=24$ mit dem korrespondierenden Prozentwert in Klammern; Angabe der kontinuierlichen Variablen als Median mit dazugehörigem IQR in eckigen Klammern.

* definiert als Mittelwert des prozentualen Verschlusses bei Vorliegen eines Thrombus in LPA + RPA. 
Tab. 19: Prädiktiver Wert ausgewählter CT-Parameter für das Erreichen des primären EP bei Patienten mit nach ESC-2014-Score intermediär-hohem Risiko.

\begin{tabular}{|c|c|c|c|}
\hline Parameter & $\begin{array}{c}\text { EP nicht erreicht } \\
(\mathrm{n}=118)\end{array}$ & $\begin{array}{c}\text { EP erreicht } \\
(n=10)\end{array}$ & p-Wert \\
\hline \multicolumn{4}{|l|}{ Thrombuslokalisation } \\
\hline Thrombus in LPA + RPA + HS & $23(19,5 \%)$ & $2(20,0 \%)$ & 1,000 \\
\hline \multicolumn{4}{|l|}{ Thrombusgröße/Thrombuslast } \\
\hline Thrombuslast LPA $>50 \%+\mathrm{RPA}>50 \%$ & $57(48,3 \%)$ & $5(50,0 \%)$ & 1,000 \\
\hline Thrombuslast LPA $>90 \%+\mathrm{RPA}>90 \%$ & $23(19,5 \%)$ & $2(20,0 \%)$ & 1,000 \\
\hline Thrombuslast LPA $=100 \%+\mathrm{RPA}=100 \%$ & $18(15,3 \%)$ & $0(0,0 \%)$ & 0,355 \\
\hline \multicolumn{4}{|l|}{ Rechter Ventrikel } \\
\hline RV-Diameter in $\mathrm{mm}$ & $48,3[43,8-54,2]$ & $52,7[40,4-57,3]$ & 0,472 \\
\hline RV/LV-Ratio & $1,22[0,98-1,50]$ & $1,23[0,97-1,73]$ & 0,576 \\
\hline $\mathrm{RV} / \mathrm{LV}$-Ratio $>1,3$ & $44(37,3 \%)$ & $4(40,0 \%)$ & 1,000 \\
\hline
\end{tabular}

Angabe der kategorialen Variablen als Anzahl n der Patienten des jeweiligen Patientenkollektivs $\mathrm{n}=118$ bzw. $\mathrm{n}=10$ mit dem korrespondierenden Prozentwert in Klammern; Angabe der kontinuierlichen Variablen als Median mit dazugehörigem IQR in eckigen Klammern.

Tab. 20: Prädiktiver Wert ausgewählter CT-Parameter für das Erreichen des primären EP bei Patienten mit nach ESC-2014-Score intermediär-niedrigem/niedrigem Risiko.

\begin{tabular}{|c|c|c|c|}
\hline Parameter & $\begin{array}{c}\text { EP nicht erreicht } \\
(n=265)\end{array}$ & $\begin{array}{c}\text { EP erreicht } \\
(n=14)\end{array}$ & p-Wert \\
\hline \multicolumn{4}{|l|}{ Thrombuslokalisation } \\
\hline Thrombus in LPA + RPA + HS & $14(5,3 \%)$ & $3(21,4 \%)$ & 0,045 \\
\hline \multicolumn{4}{|l|}{ Thrombusgröße/Thrombuslast } \\
\hline Thrombuslast LPA $>50 \%+$ RPA $>50 \%$ & $45(17,0 \%)$ & $3(21,4 \%)$ & 0,715 \\
\hline Thrombuslast LPA $>90 \%+$ RPA $>90 \%$ & $13(4,9 \%)$ & $1(7,1 \%)$ & 0,522 \\
\hline Thrombuslast LPA $=100 \%+\mathrm{RPA}=100 \%$ & $10(3,8 \%)$ & $1(7,1 \%)$ & 0,438 \\
\hline \multicolumn{4}{|l|}{ Rechter Ventrikel } \\
\hline $\mathrm{RV}$-Diameter in $\mathrm{mm}$ & $41,7[36,4-47,1]$ & $40,9[33,3-47,5]$ & 0,737 \\
\hline RV/LV-Ratio & $0,93[0,83-1,08]$ & $0,94[0,77-1,09]$ & 0,816 \\
\hline RV/LV-Ratio >1,3 & $25(9,4 \%)$ & $2(14,3 \%)$ & 0,633 \\
\hline
\end{tabular}

Angabe der kategorialen Variablen als Anzahl n der Patienten des jeweiligen Patientenkollektivs $\mathrm{n}=265$ bzw. $\mathrm{n}=14$ mit dem korrespondierenden Prozentwert in Klammern; Angabe der kontinuierlichen Variablen als Median mit dazugehörigem IQR in eckigen Klammern. 


\section{Diskussion}

Die optimierte Risikostratifizierung der akuten LE ist von besonderer Wichtigkeit, da die VTE die dritthäufigste kardiovaskuläre Erkrankung und somit einen bedeutsamen Grund für die Mortalität, Morbidität und Hospitalisation darstellt. Auch die Differenzierung des Risikoprofils insbesondere der hämodynamisch stabilen Patienten ist von Interesse, da sich hieraus verschiedene Therapieansätze ergeben (Konstantinides et al. 2014). Im diagnostischen Standardalgorithmus ist das CT als bildgebendes Mittel der Wahl verankert, weshalb sich die Auswertung seiner Bilder hinsichtlich eines möglichen Nutzens für die Risikostratifizierung anbietet. Für den klinischen Alltag ist die Identifizierung einfacher CT-Parameter - im Gegensatz zu bereits bekannten, komplizierten CT-Parametern - von besonderer Relevanz.

Das Patientenkollektiv der aktuellen Arbeit schließt 442 Patienten mit der Diagnose einer akuten LE ein. An kardiovaskulären Risikofaktoren und Vorerkrankungen fanden sich in der Studienpopulation besonders häufig eine arterielle Hypertonie, Hyperlipoproteinämie, Diabetes Mellitus, KHK oder chronische Herzinsuffizienz. Patienten des Kollektivs mit chronischer Herzinsuffizienz, vorbestehender Niereninsuffizienz sowie Synkopen, Hypotonie oder Hypoxie zum Aufnahmezeitpunkt entwickelten signifikant häufiger hämodynamisch relevante Komplikationen im kurzzeitigen Krankheitsverlauf von 30 Tagen. Insgesamt erreichten 13,1\% der Gesamtpopulation den definierten primären EP.

Die angewandten Methoden zur Bestimmung der Thrombuslast waren ebenso wie die Bestimmung der RV/LV-Ratio in der durchgeführten Interobserver- und IntraobserverUntersuchung objektiv, untersucherunabhängig und reproduzierbar. Abweichungen lagen im Interobserver-Vergleich über alle Auswerter hinweg bei $<30 \%$ bzw. $<10 \%$. Die aktuelle Arbeit erzielte - mit Ausnahme eines Messwertes - Korrelationen >0,7. Hierbei gilt eine Korrelation von 0,3-0,7 als moderater Zusammenhang und eine Korrelation von >0,7 als starker Zusammenhang zweier Ergebnisse (Geher und Hall 2014). Systematische Fehler wurden in der Bland-Altman-Analyse ausgeschlossen. Im Intraobserver-Vergleich betrugen die mittleren Abweichungen die Thrombuslast und RV/LV-Ratio betreffend $<5 \%$. Es fand sich ebenfalls eine starke Korrelation $>0,97$. 


\subsection{Thrombuslast}

Bisherige Untersuchungen der Thrombuslokalisation unterscheiden meist nur zwischen einer zentralen und einer peripheren Lage, wobei die zentrale Lokalisation alle drei Lungenhauptgefäße umfasst. In der vorliegenden Arbeit wurden HS, RPA und LPA getrennt voneinander beurteilt. In den durchgeführten Auswertungen fiel auf, dass Thrombusmaterial häufiger in der RPA als in der LPA vorkam und der prozentuale Gefäßverschluss rechts größer war, beides blieb jedoch ohne statistische Signifikanz.

Einer aktuellen Untersuchung zufolge sind zentral gelegene Thromben öfter mit klinischen und laborchemischen Markern eines erhöhten Schweregrades der akuten LE assoziiert. Zu diesen gehören RV-Dysfunktion, höhere D-Dimer-Konzentrationen im Plasma bei Diagnosestellung und eine verstärkte 15- bis 30-Tages-Mortalität (Gouin et al. 2017). Nähere Betrachtungen der Mortalität ergaben, dass eine zentrale Thrombuslokalisation ausschließlich Einfluss auf das Kurzzeitüberleben hat (Klok et al. 2008; Vedovati et al. 2013; Jain et al. 2017). Diesen Ergebnissen wird in anderer Literatur teilweise widersprochen. Dort kommt man zu dem Schluss, dass zentrale Thromben zwar mit einem schlechteren klinischen Verlauf verknüpft sind, aber keine Vorhersagekraft bezüglich der Mortalität besitzen (Meinel et al. 2015). In der aktuellen Arbeit hatten Verschlüsse der RPA und/oder LPA weder Einfluss auf die Mortalität noch auf eine klinische Verschlechterung. Allein das Vorkommen eines auf der Bifurkation reitenden HS-Thrombus erreichte das Signifikanzniveau und galt damit als Risikofaktor für das Erreichen des primären EP. Die OR lag hier bei 2,2. Diese Ergebnisse widersprechen älteren Funden, wonach ein im HS befindlicher reitender Thrombus keinen Risikofaktor für einen schwierigen klinischen Verlauf innerhalb der ersten zwei Wochen darstellt (Pruszczyk et al. 2003). Ähnlich wie bei Vedovati et al. (2012) bestand in der durchgeführten Untersuchung keine alleinige Assoziation des reitenden HS-Thrombus mit der Kurzzeit-Mortalität, weder als Folge der akuten LE noch als Folge einer anderen Ursache. Aufgrund der hohen Spezifität von 0,90 eignete sich die Thrombuslokalisation im HS vor allem zur Identifikation derjenigen Patienten ohne erhöhtes Risiko für das Auftreten hämodynamischer Komplikationen. Einschränkend muss beachtet werden, dass keine genauere Begutachtung der einzelnen Einflüsse von kardiogenem Schock, Reanimation oder Katecholaminpflichtigkeit stattfand und dass hinsichtlich vorbestehender Erkrankungen, die zu einer hämodynamischen Instabilität beitragen konnten, nicht näher differenziert wurde.

Durch Bestimmung der prozentualen arteriellen Gefäßverlegung im CT lässt sich die ungefähre Größe des LE-verursachenden Thrombus abschätzen. Damit besitzt die 
Thrombusgröße das Potential, das Ausmaß und den Schweregrad der akuten LE zu visualisieren. In der Literatur wurde vor allem der von Qanadli et al. entwickelte Obstruktionsindex hinsichtlich seines prognostischen Wertes für Komplikationen und Mortalität über verschiedene Zeitspannen hinweg untersucht. Bei einem Obstruktionsindex $>40 \%$ besteht ein 11,2-fach erhöhtes Risiko, innerhalb der ersten drei Monate nach Auftreten der LE zu versterben (van der Meer et al. 2005). Ein aus prozentualer Verlegung des Gefäßes und Thrombuslokalisation berechneter LE-Index gilt bei einem Wert $>60 \%$ als Prädiktor der Mortalität (Wu et al. 2004). Andere Untersuchungen relativieren diese Ergebnisse und stellen fest, dass der Schweregrad der arteriellen Obstruktion zwar mit dem Schweregrad der akuten LE korreliert (Collomb et al. 2003; Ghuysen et al. 2005) und sich dadurch für die Vorhersage eines klinisch schlechten Krankheitsverlaufes eignet (Meinel et al. 2015), jedoch nicht für die Vorhersage der Mortalität (Collomb et al. 2003; Ghuysen et al. 2005). CTObstruktionsindices - und damit die Okklusion - besitzen weniger Vorhersagekraft bezüglich der Kurzzeitmortalität als die Lokalisation des Gefäßverschlusses (Gouin et al. 2017). Bei Betrachtung der 30-Tages- (Araoz et al. 2007; Rodrigues et al. 2012; Furlan et al. 2012; Hefeda und Elmasry 2014), 3-Monats- (Vedovati et al. 2013) oder 12-MonatsÜberlebenszeit (Subramaniam et al. 2008) konnte in verschiedenen Studien keine signifikante Korrelation zwischen der Thrombusgröße und dem Versterben beobachtet werden. Ein möglicher Erklärungsansatz für diese fehlende Vorhersagekraft zeigt mechanische Obstruktion, vasoaktive Stoffe, reflexartige Vasokonstriktion oder systemische Hypoxämie während der akuten LE als alternative Auslöser des Gefäßverschlusses auf (El-Menyar et al. 2016). Die in der vorliegenden Arbeit durchgeführte Analyse des prognostischen Wertes der Thrombusgröße ergab, dass weder der prozentuale Gefäßverschluss von RPA, LPA noch der gemittelte Wert beider Arterien Einfluss auf die Entwicklung einer hämodynamischen Instabilität oder eines Exitus letalis innerhalb der ersten 30 Tage oder während des stationären Aufenthaltes hatten. Ebenso verhielt es sich bei einer ein- oder beidseitigen Thrombuslast $>50 \%,>90 \%,=100 \%$. Die aktuelle Arbeit schloss sich damit den Untersuchungsergebnissen an, die der Thrombusgröße in zentralen Gefäßen keinen prädiktiven Wert für die Kurzzeitmortalität oder für das Auftreten von Komplikationen bescheinigen. Vor allem die fehlende Signifikanz eines Gefäßverschlusses von LPA + RPA =100\%, die bei 7,0\% der Patienten des Studienkollektivs vorkam, legt die Vermutung nahe, dass die bewusst gewählte zweidimensionale Messung des prozentualen Gefäßverschlusses in der Transversalebene nicht ausreichend war. Hier wurde erwartet, dass die betroffenen Patienten häufiger einen hämodynamischen Kollaps erleiden oder versterben würden. Es ist daher anzunehmen, dass in einem oder beiden Pulmonalarterien noch ein gewisser Blutfluss 
herrschte, der durch die zweidimensionale Betrachtung nicht erfasst wurde. Eine genauere Untersuchung der Thrombusgröße mittels Rekonstruktion bzw. Betrachtung der Thromben in verschiedenen Körperebenen würde die Möglichkeit bieten, eine weiterführende prognostische Aussage über das tatsächliche Thrombusvolumen zu machen (Furlan et al. 2012). Dieser Untersuchungsansatz wurde aufgrund seiner Komplexität im Klinikalltag in der aktuellen Arbeit nicht weiterverfolgt. Trotz ihres eingeschränkten prognostischen Wertes kann die Thrombusgröße - zusätzlich zur standardmäßigen klinischen Beurteilung - der Beobachtung der Therapiewirksamkeit dienen (Hefeda und Elmasry 2014). Hier besteht die Möglichkeit, eine Follow-up-Untersuchung des Patientenkollektivs der aktuellen Arbeit durchzuführen und die ermittelte Thrombusgröße bei Diagnosestellung mit der Thrombusgröße während der Therapiedauer oder nach Beendigung der antikoagulativen Therapie zu vergleichen. Eine erneute Bildgebung mittels CT und eine Wiedervorstellung der Patienten wären dafür notwendig; dieses Vorgehen wird derzeit von den Leitlinien nicht empfohlen.

\subsection{Thrombuslast und Rechtsherzbelastung}

Um die Thrombuslast mit dem Auftreten einer Rechtsherzbelastung im Sinne einer RVDysfunktion zu verknüpfen, wurde stellvertretend für diese die RV/LV-Ratio betrachtet. Patienten mit zentral gelegenen Gefäßverschlüssen haben häufiger eine Rechtsherzbelastung als Patienten mit peripheren Thromben (Jain et al. 2017). Ghanima et. al fanden in ihrer Untersuchung im Jahr 2007 heraus, dass es einen signifikanten Zusammenhang zwischen zentralen Thromben und der medianen RV/LV-Ratio gibt: in dem von ihnen entwickelten Score liegt der mediane Wert bei RV/LV =1,3. Zu ähnlichen Ergebnissen kam auch die vorliegende Arbeit. Hier betrug die mediane RV/LV-Ratio bei einem Thrombus im HS + LPA + RPA 1,34. Umgekehrt fanden sich in der Untersuchung von Ghanima et al. (2007) auch bei Patienten mit einer nachgewiesenen RV-Dysfunktion in der TTE oft zentral lokalisierte Thromben. Aufgrund dieser Assoziation sollten Patienten mit zentralen Thromben keine ambulante, sondern eine stationäre antikoagulative Therapie erhalten (Senturk et al. 2017).

Die proximale Thrombusausdehnung kann ebenfalls als prognostischer Marker für eine Rechtsherzbelastung dienen (Ghanima et al. 2007). Bei Patienten mit einer RV/LV-Ratio $\geq 1$ (Furlan et al. 2012) bzw. >1,2 (El-Menyar et al. 2016) lässt sich ein signifikant größeres Thrombusvolumen messen als bei denjenigen Patienten ohne eine RV-Aufweitung. Die vorliegenden Untersuchungen zeigten ähnliches: im Falle einer Thrombuslast in LPA + 
RPA $>50 \%$ lag die mediane RV/LV-Ratio mit 1,28 vs. 0,93 höher. Gleiches ließ sich auch bei den Patienten beobachten, die den primären EP erreichten. Verschiedene Studien bescheinigen dem Obstruktionsindex nach Qanadli bei einem Gefäßverschluss $\geq 40 \%$ eine gute Korrelation mit einer RV-Dilatation: mehr als 90\% der Patienten mit RV-Dysfunktion werden identifiziert (Qanadli et al. 2001; Attia et al. 2015; El-Menyar et al. 2016). Sowohl die zentrale Thrombuslokalisation als auch die Thrombusgröße sind damit gut geeignet, um eine Rechtsherz-Dysfunktion vorherzusagen. Der genaue Cut-off der prozentualen Verlegung einzelner zentraler Gefäße sollte Inhalt fortführender Untersuchungen sein.

\subsection{RV/LV-Ratio}

In zahlreichen Studien fand sich eine signifikante Assoziation zwischen der im CT bestimmten RV/LV-Ratio und der Mortalität (Ghuysen et al. 2005), vor allem im Zeitraum des Kurzzeit-Überlebens (Schoepf et al. 2004; Ghanima et al. 2007; Furlan et al. 2012). Bei Vorliegen einer vergrößerten RV/LV-Ratio besteht ein 2,5-fach erhöhtes allgemeines Mortalitäsrisiko und ein 5-fach erhöhtes Risiko, in Folge der akuten LE zu versterben (Meinel et al. 2015). Kritische Stimmen finden hingegen keine Korrelation von RV/LV-Ratio und LE-assoziierter Mortalität (Araoz et al. 2007; El-Menyar et al. 2016). Aus einem vergrößerten RV/LV-Verhältnis ließe sich keine therapeutische Implikation ableiten (Ghanima et al. 2007). Die Inkonsistenz bezüglich der Aussagekraft der RV/LV-Ratio lässt sich möglicherweise durch die Vielfältigkeit der CT-Bildgebung bzw. der CT-Geräte (Furlan et al. 2012), durch Unterschiede in der Messung der RV/LV-Ratio (standard axial view of the heart vs. reconstructed four-chamber view) und durch eine unterschiedliche Zusammensetzung des Studienkollektivs erklären. In der vorliegenden Arbeit war die RV/LV-Ratio mit 1,15 vs. 0,98 bei hämodynamisch instabilen Patienten größer als bei stabilen, womit sich die aktuelle Untersuchung denjenigen Ergebnissen der Literatur anschließt, die die RV/LV-Ratio als für die Vorhersage der Verlaufsprognose geeignet erachten. Über den optimalen Cut-offWert für die Differenzierung von Patienten mit einem erhöhten Risiko, schwerwiegende Komplikationen der akuten LE zu erleiden, herrscht Uneinigkeit. Einige Studien ermitteln eine Trennung zwischen erhöhtem und nicht-erhöhtem Risiko bei RV/LV $\geq 0,9$ (Schoepf et al. 2004; Becattini et al. 2011). Die aktuell gültigen Leitlinien zum Management der akuten LE setzen den Cut-off auf RV/LV $\geq 0,9$ bzw. $\geq 1,0$ fest (Konstantinides et al. 2014). Patienten der vorliegenden Studienpopulation mit einer RV/LV-Ratio $>1$ wurden innerhalb des Zeitraums von 30 Tagen oder während des stationären Aufenthaltes häufiger hämodynamisch instabil. Andere Studien sahen höhere Cut-offs von $\mathrm{RV} / \mathrm{LV}=1,0$ bis 
$\mathrm{RV} / \mathrm{LV}=1,5$ als prognostisch relevant an (Reid und Murchison 1998; Collomb et al. 2003; Ghuysen et al. 2005; Furlan et al. 2012; Hefeda und Elmasry 2014; El-Menyar et al. 2016). Innerhalb des Studienkollektivs wurde mittels ROC-Analyse und Youden-IndexKlassifikation ein optimaler Cut-off-Wert von RV/LV >1,3 ermittelt. Bei Vorliegen eines RV/LV-Verhältnis 1,3:1 war das Risiko 3-fach erhöht, eine hämodynamische Instabilität oder einen Exitus letalis als Folge der akuten LE zu erleiden. Kombiniert mit einer Thrombuslast in LPA + RPA > 50\% steigerte sich das Risiko sogar auf den Faktor 4,9. Einen ähnlichen Fund beschrieben auch Vedovati et al. (2012): hier ist die OR =4,68 für schwerwiegende Komplikationen bei beidseitigen Thromben und RV-Dysfunktion. Die Sensitivität bzw. Spezifität betrug in der vorliegenden Arbeit 40\% bzw. 82\%, der PPW 25\% und der NPW 90\%. Ähnliche Ergebnisse hinsichtlich der Vorhersagewerte fanden auch andere Autoren: PPW=15,6\%, NPW=92,3\% (Schoepf et al. 2004), PPW=10,1\%, NPW=100\% (van der Meer 2005) und PPW $=10 \%, \mathrm{NPW}=100 \%$ (Becattini et al. 2011). Eine Erhöhung der RV/LV-Ratio eignet sich folglich aufgrund der niedrigen PPW nicht als alleinige Entscheidungsgrundlage für eine ausgeweitete Therapie mit reperfundierenden Maßnahmen (Schoepf et al. 2004; Becattini et al. 2011). Die hohen NPW legen hingegen nahe, dass Patienten mit einer niedrigen RV/LV-Ratio ein geringes Risiko für hämodynamische Komplikationen besitzen. Eine RV/LV-Ratio $>1,3$, wie sie in den vorliegenden Untersuchungen ermittelt wurde, zeigt eine recht deutliche Vergrößerung der rechten Herzkammer an, der auch andere kardiale Komorbiditäten zu Grunde liegen können. Lankeit (2017) schlägt vor, zukünftige Studien sollten den RV/LV-Schwellenwert für verschiedene Risikogruppen separat definieren. Ein Verhältnis von RV/LV >1 eignet sich nichtsdestotrotz als Hinweis, dass diese Patienten besonders hämodynamisch überwacht werden sollten.

\subsection{Normotensive Patienten}

Für die Risikostratifizierung der akuten LE von besonderem Interesse sind Patienten, die sich initial mit normotensiven Blutdruckwerten, aber mit erhöhtem Risiko für hämodynamische Komplikationen oder einen Exitus letalis präsentieren. Die Zahl der kreislaufstabilen Patienten betrug in der vorliegenden Arbeit 407, den primären EP erreichten hiervon 24 Patienten (5,9\%). Innerhalb der Subgruppe blieb - anders als in der Gesamtpopulation ein Thrombus im HS + LPA + RPA ohne Assoziation zum Auftreten hämodynamisch relevanter Komplikationen. Dieses Ergebnis schließt sich vorherigen Untersuchungen an, die bei Patienten mit stabilen Blutdruckwerten ebenfalls keine Prädiktion oder Auswirkung eines zentral gelegenen Thrombus auf die Mortalitätsrate fanden (Ozsu et al. 2010; Senturk 
et al. 2017). In der Literatur ist eine RV-Dilatation bei normotensiven Patienten mit einem erhöhten Risiko für das Versterben aufgrund der akuten LE assoziiert (Becattini et al. 2014). Bei einer RV/LV-Ratio $\geq 0,9$ besteht ein 1,8-fach erhöhtes Risiko der Kurzzeitmortalität (Trujillo-Santos et al. 2013). Andere Studien widersprechen diesen Funden: eine RV/LV-Ratio $\geq 0,9$ oder $\geq 1$ eigne sich nicht zur Identifikation von blutdruckstabilen Patienten mit gesteigertem Risiko für hämodynamische Komplikationen. Lediglich ein Cutoff-Wert $\geq 1,1$ könne Patienten mit nach ESC-2014-Klassifikation niedrigem Risiko in eine höhere Risikoklasse einstufen (Côté et al. 2017). Eine RV-Dilatation ist bei diesen Untersuchungen nicht mit der 30-Tages-Mortalität assoziiert, weshalb die RV/LV-Ratio nicht als Therapiegrundlage dienen sollte (Jiménez et al. 2014). Die Aussagekraft der RV/LV-Ratio ist den kritischen Stimmen zufolge gering und bringt keinen Nutzen in der klinischen Routine oder im Patientenmanagement (Coutance et al. 2011). Die Biomarker NT-proBNP ( $\geq 300 \mathrm{pmol} / \mathrm{ml})$ und Troponin $\mathrm{T}(>0,027 \mathrm{ng} / \mathrm{ml})$ allein betrachtet seien bessere prognostische Marker für die Risikostratifizierung als ihre Kombination mit einer im CT oder in der TTE bestimmten RV-Dysfunktion (Ozsu et al. 2010). In der aktuellen Arbeit waren weder der RV-Diameter noch die RV/LV-Ratio bei normotensiven Patienten, die eine hämodynamische Instabilität erlitten, signifikant vergrößert. Auch der in der Gesamtpopulation ermittelte Cut-off-Wert von RV/LV >1,3 blieb ohne Unterscheidungskraft für einen komplizierten oder unkomplizierten Krankheitsverlauf. Infolge des relativ beschränkten Platzes innerhalb des Perikards geht eine akute Erhöhung der RV-Nachlast mit einer akuten Reduktion der diastolischen LV-Dimensionen einher (Jardin et al. 1997; Wintersperger et al. 1999). Der Stellenwert der RV/LV-Ratio bei normotensiven Patienten ist folglich limitiert. Aufgrund der Möglichkeit einer anderen Einstufung der ESC-2014-Risikoklassen anhand der RV/LV-Ratio (Côté et al. 2017) sollten die bereits durch das CT gewonnen Informationen über den RV dennoch auch bei Patienten mit niedrigem Risiko für die Risikostratifizierung genutzt werden (Lankeit 2017). Weiterführende Untersuchungen könnten - wie schon in der Gesamtpopulation - auch hier auf der genauen Differenzierung der Ursache der RV-Dilatation basieren. Vorranging sollte jedoch nach anderen Markern der akuten LE gesucht werden, die normotensive Patienten mit einem erhöhten Risiko für hämodynamisch relevante Komplikationen identifizieren. 


\subsection{Chancen, Limitationen und Ausblick}

Die vorliegende Untersuchung ist aufgrund der großen Studienkohorte von 442 Patienten mit einer akuten LE und der Aktualität des fortlaufenden PERGO aussagekräftig: Die in die Auswertung eingeschlossenen Patienten erhielten ihre Diagnose im Zeitraum von 2010 bis 2016. Eine weitere Stärke des PERGO und damit auch von Vorteil für die aktuelle Arbeit war die Erhebung vielseitiger und detaillierter Patienteninformationen zum Zeitpunkt der Aufnahme: Vorerkrankungen bzw. Komorbiditäten, Risikofaktoren, Symptomatik, klinischer Status bei Diagnose und laborchemische Werte. Zusätzlich lagen - sofern durchgeführt - Befunde der TTE, des EKG und der farbkodierten Duplexsonographie vor. Für die Aufnahme in das PERGO fand keine Selektion der Patienten statt; alle Patienten mit der Diagnose einer akuten LE wurden nach persönlicher Zustimmung oder nach Zustimmung seitens des gesetzlichen Betreuers eingeschlossen. Damit ließ sich eine Verzerrung des Patientenkollektivs hinsichtlich des Schweregrades der akuten LE oder gewisser Komorbiditäten ausschließen. Eine weitere Stärke der aktuellen Untersuchung war die Anwendung einfach zu bestimmender CT-Parameter im Kontrast zu bereits bekannten, schwierig zu bestimmenden CT-Parametern wie beispielsweise den radiologischen Obstruktionsindices nach Qanadli et al. und Mastora et al.

Da es sich beim PERGO und der vorliegenden Arbeit um eine reine Beobachtungsstudie handelt, hat die Untersuchung einige Limitationen: durch das retrospektive und nichtinterventionelle Studiendesign konnte kein Einfluss auf die durchgeführte Bildgebung oder weiterführende Diagnostik genommen werden und auch die zum Diagnosezeitpunkt erhobenen klinischen Parameter konnten bei fehlenden Informationen nicht vervollständigt werden. Die CT-Bildgebung war nicht EKG-gesteuert, weshalb die Aufnahme der Bilder in verschiedenen Phasen des kardialen Kreislaufes erfolgte. Dadurch konnten die Ventrikel verschiedene Größen aufweisen (Hefeda und Elmasry 2014). Die alleinige Betrachtung der CT-Bilder schloss eine funktionale Evaluation des RV aus. Vorbestehende strukturelle Herzerkrankungen, Lungenerkrankungen und andere Komorbiditäten, die eine chronische RV-Dilatation bedingt haben könnten, wurden in den aktuellen Untersuchungen nicht differenziert betrachtet. Der Zufallsbefund einer akuten LE führte nicht zum Ausschluss aus dem Studienkollektiv. Bei der Beurteilung der Thrombuslast wurde nicht unterschieden zwischen frischen Thromben und Thrombusresiduen abgelaufener LE, die möglicherweise ebenfalls Auswirkungen auf den klinischen Status und Krankheitsverlauf des Patienten hatten. Alle Untersuchungen bezüglich des Erreichens des primären EP beschränkten sich ausschließlich auf die ersten 30 Tage oder den stationären Aufenthalt. Daten zum Lang- 
zeitüberleben wurden im PERGO zwar erhoben, doch nicht in die vorliegende Auswertung inkludiert.

Zukünftige Studien sollten weitere Möglichkeiten zur Risikostratifizierung normotensiver Patienten eruieren. Eine dreidimensionale Rekonstruktion der CT-Bilder könnte in Betracht gezogen werden und hiernach erneut der Einfluss der Thrombusgröße auf das Auftreten eines hämodynamischen Kollapses untersucht werden. Eine genauere Differenzierung der Ursachen und des Entstehungszeitpunktes der RV-Dilatation und der daraus resultierenden Rechtsherzbelastung sollte durchgeführt werden. Zusätzlich erfordert auch der Cut-off-Wert der RV/LV-Ratio weitere Validierung.

Abschließend ergab die Auswertung, dass sich im Gesamtkollektiv aller Patienten mit einer akuten LE im HS reitende Thromben für die Vorhersage eines komplizierten Krankheitsverlaufes eignen, wohingegen die prozentuale Gefäßverlegung keinen prognostischen Wert besitzt. Ein Verhältnis von $\mathrm{RV} z u \mathrm{LV}>1,3$ ist mit einem erhöhten Risiko für das Auftreten einer hämodynamischen Instabilität vergesellschaftet. Bei initial kreislaufstabilen Patienten kann keiner der genannten Marker zur Identifikation von Patienten mit einem erhöhten Risikoprofil herangezogen werden. 


\section{$5 \quad$ Zusammenfassung}

Die akute LE stellt eine häufige und schwerwiegende kardiovaskuläre Erkrankung dar, weshalb die optimale Risikostratifizierung der Patienten von großer Wichtigkeit ist. Im Rahmen der vorliegenden Arbeit sollten einfach anwendbare und reproduzierbare Messmethoden zur Bestimmung der Thrombuslast und der RV/LV-Ratio in der MDCT entwickelt werden. Besonderes Interesse galt der Identifizierung von initial normotensiven Patienten mit erhöhtem Risiko für hämodynamische Instabilitäten während des Krankheitsverlaufs. Gemessen wurde der prozentuale Gefäßverschluss von RPA und LPA. Zusätzlich fand die Betrachtung der Thrombuslokalisation im HS statt. Zur Bestimmung der RV-Dysfunktion wurde die RV/LV-Ratio herangezogen. Die Objektivität, Untersucherunabhängigkeit und Reproduzierbarkeit der Untersuchungsmethoden wurde nachgewiesen. Im Gesamtkollektiv war nur ein im HS reitender Thrombus mit einem 2,2-fach $(p=0,037)$ erhöhten Risiko für das Auftreten einer hämodynamischen Instabilität assoziiert. Die Thrombusgröße blieb ohne Einfluss auf das Erreichen des primären EP; sie eignete sich lediglich zur Vorhersage eines vergrößerten Ventrikelverhältnisses. Eine RV/LV-Ratio >1,3 war mit einem 3-fach erhöhten Risiko $(p<0,001)$ für hämodynamische Komplikationen und mit einem 3,3-fach erhöhten Risiko $(p=0,01)$ für das Auftreten eines LE-assoziierten Todes vergesellschaftet. Die Spezifität des reitenden Thrombus bzw. der RV/LV >1,3 betrug 0,90 bzw. 0,82 und 0,95 bei Kombination beider. Bei Patienten mit einer gleichzeitigen Obstruktion von $>50 \%$ in RPA + LPA und mit einer RV/LV-Ratio >1,3 stieg das Komplikationsrisiko auf das 4,9fache $(p=0,007)$. In der Gruppe der normotensiven Patienten $(n=407)$ ließ sich kein Marker für ein erhöhtes Risikoprofil identifizieren. Thrombuslokalisation und RV/LV-Ratio haben damit - besonders bei kreislaufstabilen Patienten - eine eingeschränkte Vorhersagekraft für das Auftreten hämodynamischer Instabilitäten und eignen sich nur als Zusatzuntersuchung im Rahmen der Risikostratifizierung der akuten LE. 


\title{
6 Anhang
}

\subsection{Patienteninformation und Einverständniserklärung zur Studien- teilnahme}

\author{
UNIVERSITÄTSMEDIZIN \\ GÖTTINGEN

Universitätsmedizin Göttingen, Robert-Koch-Str. 40, 37075 Göttingen

Priv.-Doz. Dr. med. C. Dellas, Abteilung Kardiologie und Pneumologie

\author{
Patienteninformation für die Studie \\ „Biomarkerbasierte Risikostratifizierung \\ von Patienten mit akuter Lungenembolie“
}

Sehr geehrte Patientin, sehr geehrter Patient,

bei Ihnen ist eine akute Lungenembolie festgestellt worden.

Bei einer akuten Lungenembolie handelt es sich um die Verstopfung einer Lungenarterie durch ein Blutgerinnsel. Kleine Gerinnsel sind nicht gefährlich und lösen sich innerhalb weniger Tage spontan auf. Bei größeren Gerinnseln kommt es jedoch zu einem Druckanstieg in den Blutgefäßen der Lunge, der zu einer Störung der Herzfunktion führen kann. In diesem Fall kann es möglicherweise zu lebensbedrohlichen Situationen kommen.

Die Risikostratifizierung dient der Abschätzung des individuellen Risikos eines Patienten, an der Lungenembolie zu versterben oder schwere Komplikationen zu entwickeln. Dies soll dann eine individuelle risikoadaptierte Therapie ermöglichen. Bisher wird diese Abschätzung durch Verfahren zur Beurteilung der Herzfunktion, wie dem Herzultraschall oder einer CT-Untersuchung sowie anhand von Laborwerten (Herztroponine) im Blut durchgeführt. Diese Befunde können Hinweise darauf geben, dass ein erhöhtes Risiko besteht. Die genannten Verfahren unterliegen jedoch einigen Einschränkungen, so dass die Notwendigkeit zur Untersuchung neuer Biomarker für eine optimierte Risikostratifizierung besteht.

$\underline{\text { Wir möchten Sie daher bitten, an dieser wissenschaftlichen Studie freiwillig teilzunehmen. }}$

Ziel der Studie ist es, neue Biomarker (Laborwerte) im Blut hinsichtlich ihrer Eignung für eine optimierte Risikostratifizierung von Patienten mit einer akuten Lungenembolie zu untersuchen. Die an der Studie beteiligten Ärzte erhoffen sich damit, in Zukunft eine Verbesserung der medizinischen Versorgung von Patienten mit einer akuten Lungenembolie erreichen zu können.

Studiendurchführung: Weitere studienbedingte Untersuchungen sind nicht vorgesehen. Wir werden uns nach 6 Monaten (sowie ggf. erneut zu einem späteren Zeitpunkt) telefonisch mit Ihnen oder Ihrem Hausarzt in Verbindung setzen, um den Verlauf Ihrer Erkrankung zu erfragen. 
Risiken: Diese Studie dient ausschließlich der Erhebung von patientenbezogenen Daten (sogenannte epidemiologische Studie). Eine therapeutische Intervention (z.B. durch die Gabe einer Prüfmedikation) ist nicht vorgesehen. Die Studie hat keinen Einfluss auf Ihre Behandlung und die medizinische Betreuung liegt ausschließlich im Ermessen des behandelnden Arztes. Die im Rahmen der Studie gemessenen Biomarker werden den behandelnden Ärzten nicht mitgeteilt und zu keinem Zeitpunkt verwendet, um den Therapieerfolg zu überwachen oder Therapieentscheidungen zu beeinflussen.

Durch die Studienteilnahme kann für Sie durch die zusätzlichen Blutentnahmen ein geringes Verfahren-spezifisches zusätzliches Risiko bestehen. Dazu zählen das Auftreten von blauen Flecken im Bereich der Einstichstelle und ein geringes Risiko für lokale oder allgemeine Infektionen. Sehr selten kann eine Verletzung eines Hautnervens auftreten, die evtl. einen chronischen Verlauf nehmen kann.

Vorteile: Durch die Studienteilnahme besteht für Sie kein unmittelbarer Nutzen.

Vertraulichkeit und Datenschutz: Die an der Studie beteiligten Ärzte und Medizinstudenten unterliegen grundsätzlich der ärztlichen Schweigepflicht. Für die Studie relevante personenbezogene Daten, insbesondere medizinische Befunde, werden durch Befragung von Ihnen, Ihrem Hausarzt und Ihren betreuenden Ärzten sowie durch Einsicht in Ihre Krankenakte erhoben. Die elektronische Speicherung und Auswertung Ihrer Daten sowie die Archivierung Ihrer Blutproben erfolgt pseudonymisiert (also durch Verschlüsselung mit einem Nummerncode). Die Zuordnung von Daten oder Laborwerten aus den Blutproben zu einer Person ist nur durch den Einsatz des Schlüssels möglich, der für die Pseudonymisierung verwendet wurde. Die personenbezogenen Daten und die Blutproben werden unter besonderen Schutzvorkehrungen getrennt von den pseudonymisierten Daten aufbewahrt. Eine Entschlüsselung ist nur durch die verantwortlichen Studienärzte möglich. Eine Weitergabe der Daten ist nicht vorgesehen. Die Speicherung der Patientendaten und Identifizierungsschlüssel erfolgt für 15 Jahre, die Aufbewahrung der Blutproben 10 Jahre. Ergebnisse der Studie sollen in medizinischen oder wissenschaftlichen Zeitschriften publiziert werden, Sie bleiben als Patient dabei jedoch anonym. Sollten Sie Ihre Studienteilnahme widerrufen, werden die gespeicherten Patientendaten gelöscht und die gelagerten Blutproben verworfen (entsorgt).

Studienteilnahme und Widerrufsmöglichkeit: Die Teilnahme an dieser Studie ist freiwillig und Sie können jederzeit ohne Angabe von Gründen von der Teilnahme zurücktreten. Wenn sie sich entscheiden sollten, an dieser Studie nicht teilzunehmen oder Ihre Teilnahme widerrufen, wird dies keinen Einfluss auf Ihre medizinische Betreuung oder das Verhältnis zwischen Ihnen und den behandelnden Ärzten haben.

Göttingen, August 2010

Priv.-Doz. Dr. med. C. Dellas

Studienleiterin (Tel.: 39-12575)
Dr. med. M. Lankeit

Studienärztin (Tel.: 39-12575) 
Universitätsmedizin Göttingen, Robert-Koch-Str. 40, 37075 Göttingen

Priv.-Doz. Dr. med. C. Dellas, Abteilung Kardiologie und Pneumologie

\author{
Einverständniserklärung zur Studie \\ „Biomarkerbasierte Risikostratifizierung \\ von Patienten mit akuter Lungenembolie“
}

Hiermit bestätige ich, dass ich von der unterzeichnenden Ärztin/dem unterzeichnenden Arzt über die Art, die Bedeutung, Risiken und Nutzen sowie den Umfang der o.g. wissenschaftlichen Studie aufgeklärt wurde. Ich habe die Patienteninformation gelesen und verstanden und hatte Gelegenheit, Fragen zu stellen. Ich habe eine Kopie der Patienteninformation und dieser Einverständniserklärung erhalten. Ich wurde darüber informiert, dass meine Teilnahme an der o.g. wissenschaftlichen Studie für mich mit einem geringen Risiko durch zusätzliche Blutabnahmen verbunden sein kann und mit keinem direkten Nutzen verbunden ist.

Ich hatte ausreichend Zeit, mich zur Teilnahme an o.g. wissenschaftlicher Studie zu entscheiden und meine Einwilligung erfolgt freiwillig. Ich weiß, dass ich jederzeit und ohne Angabe von Gründen diese Zustimmung widerrufen kann, ohne dass sich dieser Entschluss nachteilig auf meine ärztliche Behandlung oder medizinische Betreuung auswirken wird.

Mir ist bekannt und ich bin damit einverstanden, dass personenbezogene Daten, die über mich erhoben werden und Blutproben, die von mir archiviert werden, in verschlüsselter Form (pseudonymisiert) gespeichert und ausgewertet werden. Ich stimme der Aufbewahrung der personenbezogenen Daten (Patientendaten und Identifizierungsschlüssel für 15 Jahre, Blutproben für 10 Jahre) zu. Ich wurde darüber informiert und ich gebe mein Einverständnis, dass die Entschlüsselung der Patientendaten nur durch die verantwortlichen Studienärzte möglich und keine Weitergabe der Daten vorgesehen ist.

Hiermit erkläre ich meine freiwillige Einwilligung zu einer Teilnahme an der o.g. wissenschaftlichen Studie. Ich bin mit der Datenerhebung, der pseudonymisierten Verarbeitung und Weitergabe der Daten in beschriebener Art und Weise einverstanden.

den

Ort, Datum den

Ort, Datum $\overline{\text { Unterschrift der Patientin / des Patienten }}$

Unterschrift der aufklärenden Ärztin / des aufklärenden Arztes 


\subsection{Datenerfassungsbogen für das Pulmonary Embolism Registry Göttingen}

\section{Patientendaten}

Geburtsdatum:

Aufnahmedatum:

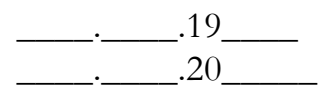

Größe: $\mathrm{cm}$
Geschlecht: $\mathrm{m} \square \mathrm{w} \square$

Entlassungs-/Todesdatum:

(entlassen von Station $\mathrm{kg} \quad$ BMI:

Hausarzt:

Aufklärung über FU: ja $\square$ nein $\square$;

einverstanden: ja $\square$ nein $\square$

Telefonnr. privat:

\section{Teilnahme an klinischen Studien}

Der Patient wurde außerdem in eine klinische Studie eingeschlossen $\quad$ ja $\square$ nein $\square$ wenn ja, welche Studie:

\section{Initiale Risikostratifizierung (nach ESC-Leitlinie 2014)}

$$
\text { Einteilung*: Hoch-Risiko } \square \text { Nicht-Hoch-Risiko } \square
$$

*Definition:

- Die Risikostratifizierung erfolgt anhand des Blutdrucks bei Aufnahme bzw. vor Beginn einer Therapie.

- Als Hoch-Risiko Patienten werden Patienten definiert, die einen systolischen RR $<90 \mathrm{mmHg}$ bzw. einen Blutdruckabfall um $\geq 40 \mathrm{mmHg}$ in $>15 \mathrm{~min}$ erleiden, insofern dieser nicht durch eine neu-aufgetretene Arrhythmie, Hypovolämie oder Sepsis erklärbar ist. Dazu zählen auch alle Patienten, die vor Beginn einer Therapie reanimiert wurden bzw. eine prähospitale Lyse erhalten haben.

- $\quad$ Nicht-Hoch-Risiko sind alle Patienten mit einem syst. RR $\geq 90 \mathrm{mmHg}$

\section{Weiterführende Risikostratifizierung (nach ESC-Leitlinie 2014)}

Einteilung*: Niedriges-Risiko $\square \quad$ Intermediär-niedriges Risiko $\square \quad$ Intermediär-hohes Risiko $\square$

*Definition:

2: intermediär-hohes Risiko (ALLE 3 Kriterien müssen erfüllt sein!)

a) $\mathrm{sPESI} \geq 1$ Punkte UND

b) RHB (im Echo oder RV/LV ratio $\geq 1$ im CT) UND

c) Biomarkererhöhung (Troponin Erhöhung (cTnT $\geq 0,03 \mu \mathrm{g} / \mathrm{l}$, hs'TnT $\geq 14 \mathrm{pg} / \mathrm{ml}, \mathrm{cTnI} \geq 13 \mathrm{ng} / \mathrm{l}$ ㅇ / $\geq 33 \mathrm{ng} / \mathrm{l}$ 3); NT-proBNP $\geq 600 \mathrm{pg} / \mathrm{ml}$ oder BNP $\geq 100 \mathrm{pg} / \mathrm{ml}$ )

3: intermediär-niedriges Risiko (1-2 der 3 Kriterien müssen erfüllt sein)

a) $\quad$ PESI $\geq 1$ Punkte ODER

b) RHB (im Echo oder RV/LV ratio $\geq 1$ im CT) ODER

c) Biomarkererhöhung (Troponin Erhöhung ( $\operatorname{c} \operatorname{Tn} T \geq 0,03 \mu \mathrm{g} / \mathrm{l}$, hs'TnT $\geq 14 \mathrm{pg} / \mathrm{ml}, \mathrm{c} \operatorname{TnI} \geq 13 \mathrm{ng} / 1$ q / $\geq 33 \mathrm{ng} / \mathrm{l}$ đ); NT-proBNP $\geq 600 \mathrm{pg} / \mathrm{ml}$ oder BNP $\geq 100 \mathrm{pg} / \mathrm{ml}$ )

4: niedriges Risiko (KEINES der 3 Kriterien darf erfüllt sein!)

Klinische Wahrscheinlichkeit einer LE: Wells Score

\begin{tabular}{|c|c|c|c|}
\hline Klinische Charakteristik & ja & nein & Score \\
\hline 1. Klinische Charakteristik einer TVT & & & 3,0 \\
\hline 2. LE wahrscheinlicher als andere Diagnose & & & 3,0 \\
\hline 3. Herzfrequenz $>100 / \mathrm{min}$. & & & 1,5 \\
\hline 4. Immobilisation oder OP (in den vergangenen 4 Wochen) & & & 1,5 \\
\hline 5. Frühere TVT oder LE & & & 1,5 \\
\hline 6. Hämoptysen & & & 1,0 \\
\hline 7. Krebserkrankung (aktiv oder in den vergangenen 6 Monaten) & & & 1,0 \\
\hline Wahrscheinlichkeit einer LE & \multicolumn{3}{|c|}{ Score } \\
\hline gering $(<2,0)=1 / \operatorname{mittel}(2,0-6,0)=2 / \operatorname{hoch}(>6,0)=3$ & & & \\
\hline
\end{tabular}


Klinische Wahrscheinlichkeit einer LE: Revidierter Genfer Score

\begin{tabular}{|c|c|c|c|}
\hline Klinische Charakteristik & ja & nein & Score \\
\hline 1. Alter $>65$ Jahre & & & +1 \\
\hline 2. Frühere TVT oder LE & & & +3 \\
\hline 3. OP oder Knochenfraktur innerhalb des letzten Monats & & & +2 \\
\hline 4. aktive Krebserkrankung & & & +2 \\
\hline 5. Symptom: einseitiger Beinschmerz & & & +3 \\
\hline 6. Symptom: Hämoptyse & & & +2 \\
\hline 7. Herzfrequenz (bei Aufnahme/Diagnosestellung) 75-94/min & & & +3 \\
\hline 8. Herzfrequenz (bei Aufnahme/Diagnosestellung) $\geq 95 / \mathrm{min}$ & & & +5 \\
\hline 9. Schmerzen entlang tiefer Beinvene, einseitiges Ödem & & & +4 \\
\hline Wahrscheinlichkeit einer LE & \multicolumn{3}{|c|}{ Score } \\
\hline
\end{tabular}

\section{Pulmonary Embolism Severity Index (PESI) Score}

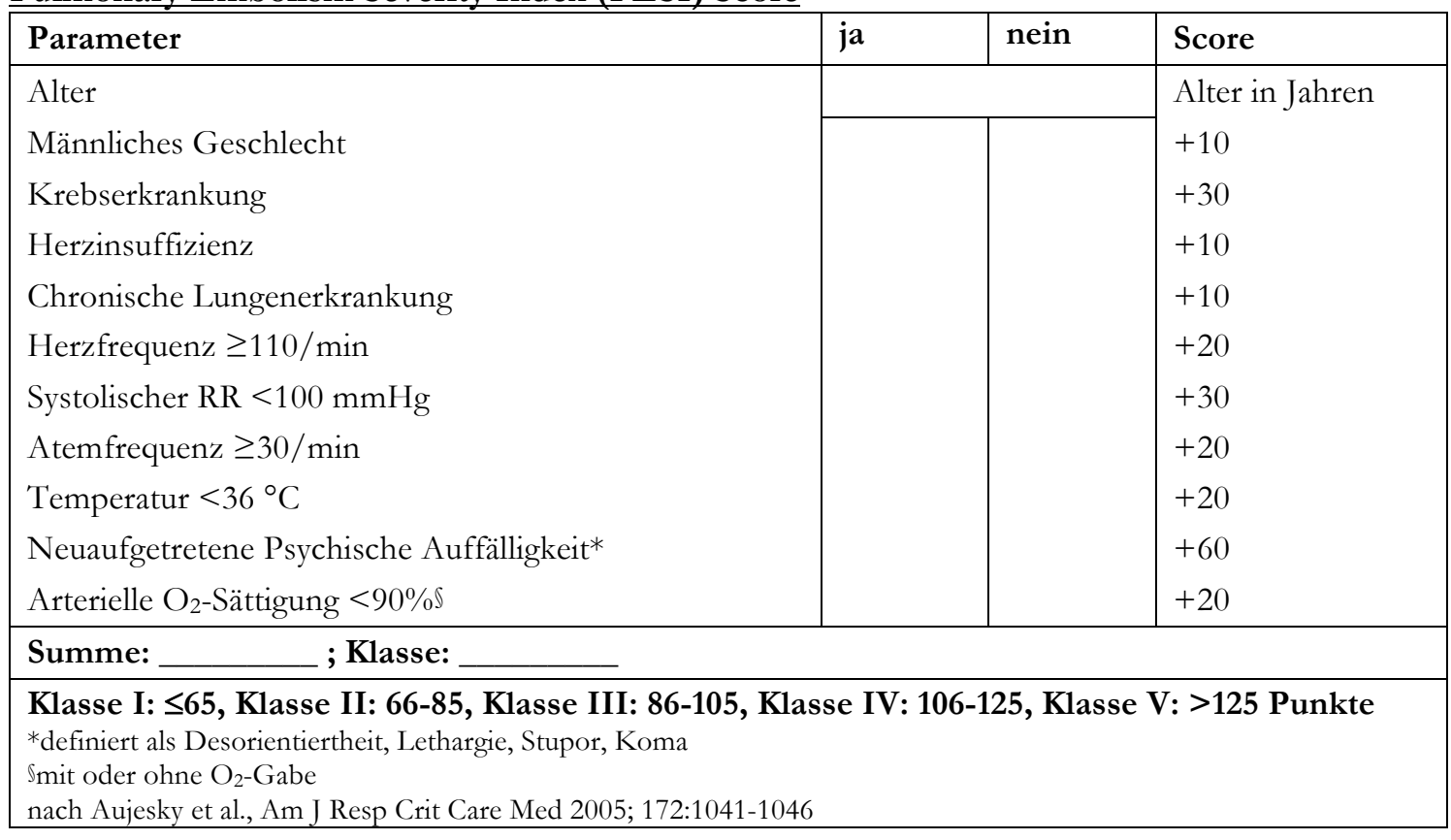

simplifizierter PESI (sPESI) Score

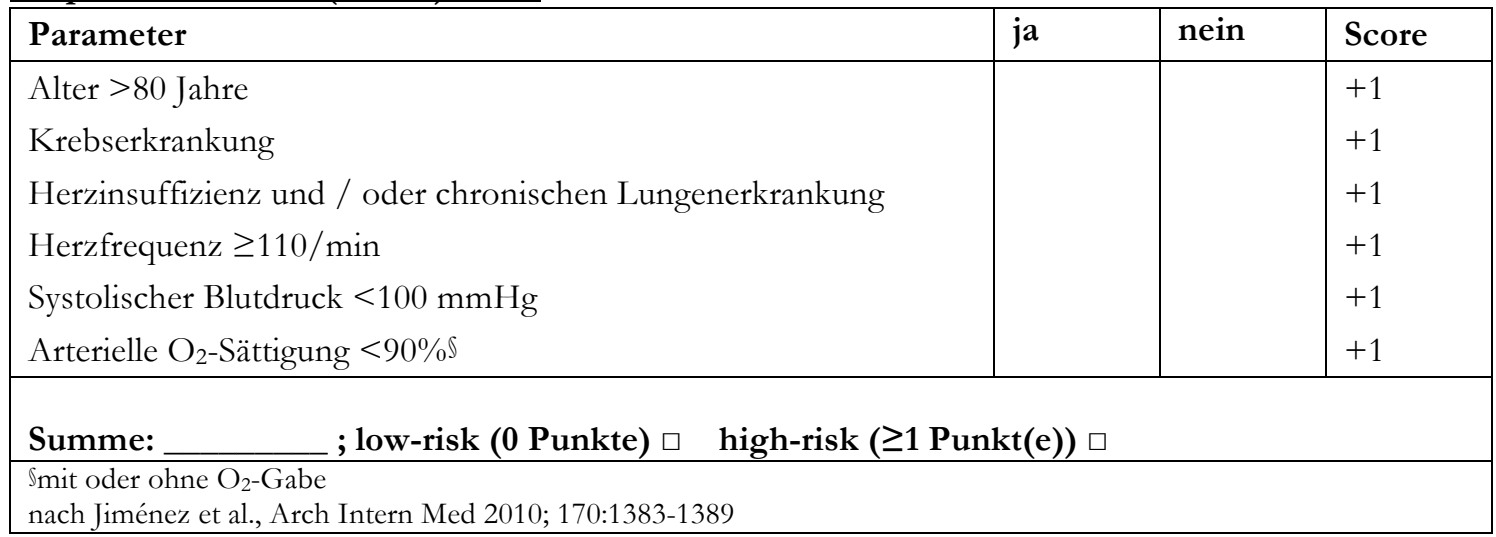


Bova Score (LE-bedingte Komplikationen bei normotensiven Patienten)

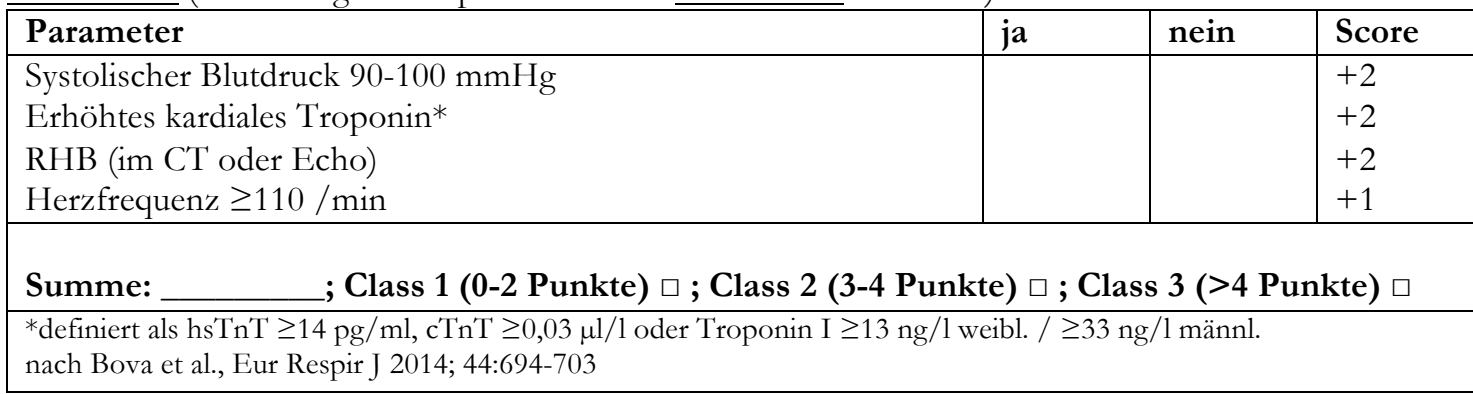

Modifizierter FAST Score (LE-bedingte Komplikationen bei normotensiven Patienten)

\begin{tabular}{|l|l|l|l|}
\hline \hline Parameter & ja & nein & Score \\
\hline $\begin{array}{l}\text { Erhöhtes kardiales Troponin* } \\
\text { Synkope }\end{array}$ & & & $+1,5$ \\
Herzfrequenz $\geq 100 / \mathrm{min}$ & & $+1,5$ \\
\hline & & +2 \\
Summe: $\quad$ low-risk (<3 Punkte) $\square \quad$ intermediate-high-risk ( $\geq 3$ Punkte) \\
\hline $\begin{array}{l}\text { *definiert als hsTnT } \geq 14 \mathrm{pg} / \mathrm{ml}, \mathrm{cTnT} \geq 0,03 \mu \mathrm{l} / \mathrm{l} \text { oder Troponin I } \geq 13 \text { ng/l weibl. / } \geq 33 \text { ng/l männl. } \\
\text { nach Hobohm et al., unpublished }\end{array}$
\end{tabular}

VTE-BLEED Score (Blutungsscore)

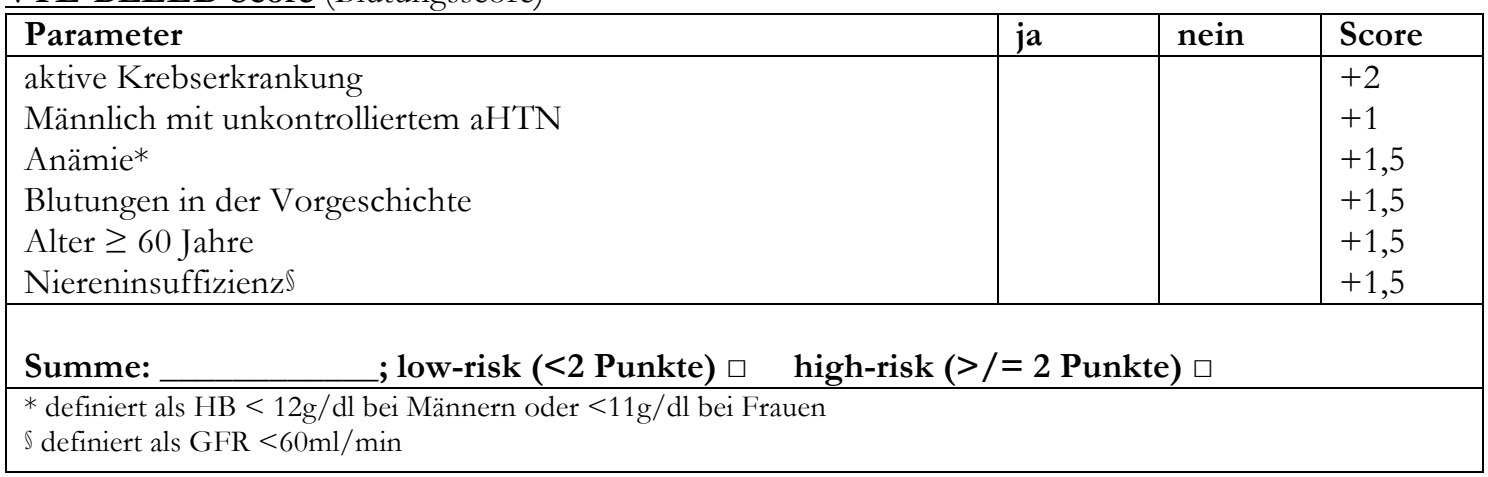

\section{$\underline{\text { Anamnese }}$}

\section{Risikofaktoren für LE}

Unprovozierte* Lungenembolie

ja $\square$ nein $\square$

*definiert als keine OP; kein Trauma; keine Immobilisierung; keine Schwangerschaft/postpartum, keine Kontrazeptiva/Hormontherapie

Z.n. tiefer Beinvenenthrombose

Z.n. Lungenembolie

ja $\square$ nein $\square$; wann

Hospitalisierung (innerhalb der letzten 3 Monate) ja $\square$ nein $\square$;

$\square$ Myokardinfarkt $\square$ kardiolog. Erkrankung $\square$ internistische Erkrankung; sonstige

Z.n. OP (innerhalb der letzten 4 Wochen)

ja $\square$ nein $\square$

Z.n. Trauma (innerhalb der letzten 4 Wochen) $\quad$ ja $\square$ nein $\square$

Immobilisierung (innerhalb der letzten 4 Wochen) ja $\square$ nein $\square$

Reise

ja $\square$ nein $\square$

Nikotin

Port / ZVK / SM / ICD

ja $\square$ nein $\square$; py bis wann

Gravidität / postpartum (6 Wochen)

bekannte Hyperkoagulabilität

Krebserkrankung

Metastasen

Aktuelle Chemotherapie

ja $\square$ nein $\square$; welche

ja $\square$ nein $\square$; SSW

ja $\square$ nein $\square$; welche

ja $\square$ nein $\square$; welche

ja $\square$ nein $\square$

ja $\square$ nein $\square$ 


\section{Vorerkrankungen}

Herzinsuffizienz

Koronare Herzkrankheit

Z. n. ACS

COPD / Asthma / PAH

Vorhofflimmern

Arterielle Hypertonie pAVK

Z.n. Apoplex / PRIND / TIA

Hyperlipoproteinämie

Diabetes mellitus

Niereninsuffizienz

Lebererkrankung (Fibrose, Hepatitis)

Alkoholabusus

Z.n. Blutung (z.B. GI Blutung)

Erhöhte Sturzneigung

Chron. inflamm. Darmerkrankung

Rheumatoide Erkrankung ja $\square$ nein $\square$

ja $\square$ nein $\square$

ja $\square$ nein $\square$; wann

ja $\square$ nein $\square$; welche

ja $\square$ nein $\square$; wann

ja $\square$ nein $\square$

ja $\square$ nein $\square$

ja $\square$ nein $\square$; wann

ja $\square$ nein $\square$

ja $\square$ nein $\square$; Typ

ja $\square$ nein $\square$; akut $\square$ chron $\square$

ja $\square$ nein $\square$; welche

ja $\square$ nein $\square$

ja $\square$ nein $\square$; welche

ja $\square$ nein $\square$

ja $\square$ nein $\square$; welche

ja $\square$ nein $\square$; welche

ja $\square$ nein $\square$; welche

ja $\square$ nein $\square$; welche

Kontrazeptiva / Hormonpräparate therapeutische Antikoagulation

$\square$ VKA $\square$ Rivaroxaban $\square$ Apixaban $\square$ Dabigatran $\square$ Edoxaban $\square$ NMH $\square$ Fondaparinux $\square$ UFH

prophylaktische Antikoagulation ja $\square$ nein $\square$; welche

ASS

$\square$ Rivaroxaban $\square$ Apixaban $\square$ Dabigatran $\square$ Edoxaban $\square$ NMH $\square$ Fondaparinux $\square$ UFH

ja $\square$ nein $\square$

thrombozytäre ADP P2Y12 Inhibitoren ja $\square$ nein $\square$; welche

Cortison / Immunsupp. $\quad$ ja $\square$ nein $\square$

NSAR

Statin

Sonstige:

ja $\square$ nein $\square$; welche

ja $\square$ nein $\square$; welche

\section{Symptomatik}

Beginn der Symptomatik: $\square$ keine $\square$ innerhalb 24h $(\tau$ h vor Aufnahme) $\square 1-4 \mathrm{~d} \square 4-14 \mathrm{~d} \square>14 \mathrm{~d}$

Beinschwellung

Beinschmerzen

thorakale Schmerzen

Dyspnoe

Hämoptysen

Synkope

Herzrhythmusstörungen

ja $\square$ nein $\square$

ja $\square$ nein $\square$

ja $\square$ nein $\square$

ja $\square$ nein $\square$

ja $\square$ nein $\square$

ja $\square$ nein $\square$

kardiogener Schock (nach Definition)

Herzstillstand / Reanimation

reduzierter mentaler Zustand

ja $\square$ nein $\square$; supraventrikulär $\square$ ventrikulär $\square$

ja $\square$ nein $\square$

ja $\square$ nein $\square$

ja $\square$ nein $\square$

(Desorientierung, Lethargie, Koma, Somnolenz, Stupor)

Apoplex / PRIND / TIA ja $\square$ nein $\square$

paradoxe arterielle Embolie $\quad$ ja $\square$ nein $\square$

\section{$\underline{\text { Klinischer Status bei Diagnose }}$}

Puls $/ \min (\square \mathrm{NA})$

Atemfrequenz _ $/ \min (\square \mathrm{NA})$

$\mathrm{SaO}_{2} \_\%$ obne $\mathrm{O}_{2}$-Gabe $(\square \mathrm{NA})$

$\mathrm{PO}_{2}$ (art.) _ $\mathrm{mmHg}$ obne $\mathrm{O}_{2}$-Gabe

$\mathrm{PCO}_{2}$ (art.) $\mathrm{mmHg}$
$\mathrm{RR} \_$_ _ $\mathrm{mmHg}(\square \mathrm{NA})$

Temperatur $\_{ }^{\circ} \mathrm{C}$

$\mathrm{SaO}_{2} \_\%$ unter $\quad 1 \mathrm{O}_{2}(\square \mathrm{NA})$

$\mathrm{PO}_{2} \_\mathrm{mmHg}$ unter ${ }_{1} \mathrm{O}_{2}$ 


\section{$\underline{\text { Diagnostik }}$}

Echokardiographie (Befund kopieren und archivieren)

durchgeführt

RHB (nach Definition)

RV-Dilatation $(\mathrm{RV}>\mathrm{LV})$

RVD1,2,3(<42,35,86)

RVEDD:

LVEDD:

paradoxe Septumbewegung

LV-EI / D-sign

VCI gestaut

intrakardiale Thromben

PFO

TR-Jet Geschwindigkeit:

TI

geschätzter PAPsys:

TAPSE

PV Acc'T

$\mathrm{S}^{\prime}$

diastolische Dysfunktion

$\square$ normal (keine diastolische Dysfunktion)

$\square$ Pseudonormales EA Muster (Grad 2)

$\square$ nicht bestimmbar (z.B. bei VHF)

LV-Funktion

alternativ:

normal $\square \quad$ eingeschränkt $\square \mathrm{EF}$

leichtgradig $\square /$ mittelgradig $\square$ / hochgradig $\square$ eingeschränkt ja $\square$ nein $\square$; Datum, Ubrzeit.

ja nein $\square$

ja $\square$ nein $\square$

$\mathrm{mm}$ (bis $30 \mathrm{~mm}$ normal)

$\mathrm{mm}$

ja $\square$ nein $\square$

ja $\square$ nein $\square$

ja $\square$ nein $\square$

ja $\square$ nein $\square$; wenn ja, T'TE $\square$ TEE $\square$ CT $\square$

ja $\square$ nein $\square$ $\mathrm{m} / \mathrm{s}$

ja $\square$ nein $\square$; Grad ${ }^{\circ}\left(\mathrm{MI} \_{ }^{\circ}, \mathrm{AI} \_{ }^{\circ}\right)$ $\mathrm{mmHg}$ $\mathrm{mm}$

$\mathrm{ms}$

$\mathrm{cm} / \mathrm{s}$

$\square$ Relaxationsstörung (Grad 1)

$\square$ Restriktives EA Muster (Grad 3)

Elektrokardiogramm (EKG) (bei Aufnahme, Befund kopieren und archivieren)

$\mathrm{S}_{\mathrm{I}} \mathrm{Q}_{\text {III- }}$-Typ

T-Negativierung $\mathrm{V}_{1}-\mathrm{V}_{3}$

Rechtsschenkelblock

atriale Arrhythmien

nicht verwertbar (LSB, SM)

LE bzw. TVT gesichert durch (Befund kopieren und archivieren)

Pulmonalisangiographie ja $\square$ nein $\square$

Kompressions-Sonographie $\quad$ ja $\square$ nein $\square$; TVT ja $\square$ nein $\square$; wo

Phlebographie

Lungenszintigramm

ja $\square$ nein $\square$; TVT ja $\square$ nein $\square$; wo

ja $\square$ nein $\square$; Segmente

Spiral-CT (Befund kopieren, archivieren und in Kommentar eintragen)

durchgeführt

ja $\square$ nein $\square$; Datum, Uhrzeit.

Diagnose:

$\begin{array}{ll}\text { CT Venen } & \text { ja } \square \text { nein } \square \text {; TVT ja } \square \text { nein } \square \text {; wo } \\ \text { RHB } & \text { ja } \square \text { nein } \square\end{array}$

Thrombophiliediagnostik (Befund kopieren und archivieren)

durchgeführt

ja $\square$ nein $\square$

Diagnose: 
Komplikationen (während des stationären Aufenthaltes oder innerhalb der ersten 30 Tage)

Rezidiv-LE / TVT

Sepsis / Infarktpneumonie ANV / MOV

Apoplex / PRIND / TIA paradoxe arterielle Embolie Blutungen

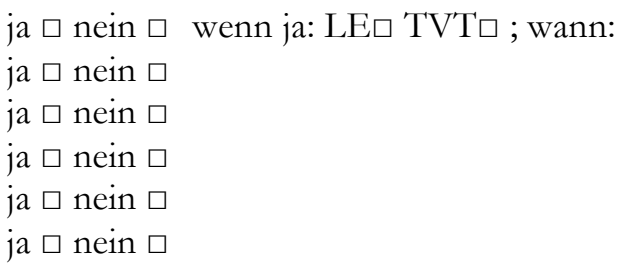

$\square$ 1a - severe bleeding (tödlich, lebensbedrohlich, mit hämodynamischer Instabilität und Notfalltransfusion / OP, Katecholamine, jede intrakranielle Blutung)

$\square 1 \mathrm{~b}$ - moderate bleeding (Notwendigkeit von EKs, jedoch keine Lebensgehfahr oder hämodynamische Instabilität)

$\square 2$ - minor bleeding (Hb-relevant ( $\mathrm{g} / \mathrm{dl})$ Einblutung / Blutverluste ohne EK Substitution)

Auftreten (Datum, Ubrzeit. ___ _ ggf. Stunden nach Lyse h

Anzahl der substituierten EKs:

Katecholamingabe

Intubation

Reanimation

Tod

Obduktion ja $\square$ nein $\square$ (außer Dopamin $\leq 5$ Gamma)

ja $\square$ nein $\square$ Anzahl der Tage:

ja $\square$ nein $\square$ neuroprotektive Kühlung ja $\square$ nein $\square$

ja $\square$ nein $\square$ wenn ja: Datum:

ja $\square$ nein $\square$ Todesursache:

\section{Therapie}

Thrombolyse

ja $\square$ nein $\square$

Datum, Uhrzeit:

Thrombusdefragmentation ja $\square$ nein $\square$; Datum, Uhrzeit: operative Thrombektomie Cava Schirm ja $\square$ nein $\square$; Datum, Uhrzeit:

Heparingabe vor BE ZP1 ja $\square$ nein $\square$ ja $\square$ nein $\square$; wenn ja, wann:

$\square$ UFH $\square \mathrm{NMH} /$ Fondaparinux $\square$ Rivaroxaban $\square$ Apixaban $\square$ Aspirin i.v.

$\square$ loading dose P2Y12 Inhibitor: Substanz: $\square$ sonstige:

Dauer der Heparingabe (UFH, NMH) vor Wechsel auf VKA / NOAK: therapeutische AK (initial) ja $\square$ nein $\square$; wenn ja:

$\square \mathrm{UFH} \square \mathrm{NMH} /$ Fondaparinux $\square$ Rivaroxaban $\square$ Apixaban $\square$ sonstige: therapeutische AK (Entlassung) ja $\square$ nein $\square$; wenn ja:

$\square$ VKA $\square$ Rivaroxaban $\square$ Apixaban $\square$ Dabigatran $\square$ Edoxaban $\square$ NMH wenn keine therapeutische Antikoagulation, bitte in Kommentar schreiben warum!!!

\section{Kopieren und archivieren:}

- Labor (gesamter Aufenthalt) + Thrombophiliediagnostik

- radiologische / nuklearmedizinische Bildgebung

- Echokardiographie, EKG, Herzkatheter Bericht, sonstige Befunde; Entlassungsbrief Notarzt Protokoll, Aufnahmebogen (initial), Stammdatenblatt, ggf. Pflegekurven 


\section{$7 \quad$ Literaturverzeichnis}

Agnelli G, Becattini C (2015): Anticoagulant treatment for acute pulmonary embolism: a pathophysiology-based clinical approach. Eur Respir J 45, 1142-1149

Anderson FA, Spencer FA (2003): Risk Factors for Venous Thromboembolism. Circulation 107, I-9-I16

Apfaltrer P, Henzler T, Meyer M, Roeger S, Haghi D, Gruettner J, Süselbeck T, Wilson RB, Schoepf UJ, Schoenberg SO, Fink C (2012): Correlation of CT angiographic pulmonary artery obstruction scores with right ventricular dysfunction and clinical outcome in patients with acute pulmonary embolism. Eur J Radiol 81, 2867-2871

Araoz PA, Gotway MB, Harrington JR, Harmsen WS, Mandrekar JN (2007): Pulmonary Embolism: Prognostic CT Findings. Radiology 242, 889-897

Attia NM, Seifeldein GS, Hasan AA, Hasan A (2015): Evaluation of acute pulmonary embolism by sixtyfour slice multidetector CT angiography: Correlation between obstruction index, right ventricular dysfunction and clinical presentation. Egypt J Radiol Nucl Med 46, 25-32

Aujesky D, Obrosky DS, Stone RA, Auble TE, Perrier A, Cornuz J, Roy P-M, Fine MJ (2005): Derivation and Validation of a Prognostic Model for Pulmonary Embolism. Am J Respir Crit Care Med 172, 1041-1046

Becattini C, Agnelli G, Vedovati MC, Pruszczyk P, Casazza F, Grifoni S, Salvi A, Bianchi M, Douma R, Konstantinides S, et al. (2011): Multidetector computed tomography for acute pulmonary embolism: diagnosis and risk stratification in a single test. Eur Heart J $\underline{32}, 1657-1663$

Becattini C, Agnelli G, Germini F, Vedovati MC (2014): Computed tomography to assess risk of death in acute pulmonary embolism: a meta-analysis. Eur Respir J 433, 1678-1690

Berghaus TM, Haeckel T, Behr W, Wehler M, von Scheidt W, Schwaiblmair M (2010): Central thromboembolism is a possible predictor of right heart dysfunction in normotensive patients with acute pulmonary embolism. Thromb Res 126, e201-e205

Ceriani E, Combescure C, Le Gal G, Nendaz M, Perneger T, Bounameaux H, Perrier A, Righini M (2010): Clinical prediction rules for pulmonary embolism: a systematic review and meta-analysis. J Thromb Haemost $\underline{8}$, 957-970

Chew HK, Wun T, Harvey D, Zhou H, White RH (2006): Incidence of Venous Thromboembolism and Its Effect on Survival Among Patients With Common Cancers. Arch Intern Med 166, 458-464

Cohen AT, Agnelli G, Anderson FA, Arcelus JI, Bergqvist D, Brecht JG, Greer IA, Heit JA, Hutchinson JL, Kakkar AK, et al. (2007): Venous thromboembolism (VTE) in Europe. The number of VTE events and associated morbidity and mortality. Thromb Haemost 98, 756-764

Collomb D, Paramelle PJ, Calaque O, Bosson JL, Vanzetto G, Barnoud D, Pison C, Coulomb M, Ferretti $G$ (2003): Severity assessment of acute pulmonary embolism: evaluation using helical CT. Eur Radiol $\underline{13}, 1508-1514$

Côté B, Jiménez D, Planquette B, Roche A, Marey J, Pastré J, Meyer G, Sanchez O (2017): Prognostic value of right ventricular dilatation in patients with low-risk pulmonary embolism. Eur Respir J $\underline{50,}$ 1701611

Coutance G, Cauderlier E, Ehtisham J, Hamon M, Hamon M (2011): The prognostic value of markers of right ventricular dysfunction in pulmonary embolism: a meta-analysis. Crit Care 15, R103

Cushman M, Tsai AW, White RH, Heckbert SR, Rosamond WD, Enright P, Folsom AR (2004): Deep vein thrombosis and pulmonary embolism in two cohorts: the longitudinal investigation of thromboembolism etiology. Am J Med 117, 19-25

El-Menyar A, Nabir S, Ahmed N, Asim M, Jabbour G, Al-Thani H (2016): Diagnostic implications of computed tomography pulmonary angiography in patients with pulmonary embolism. Ann Thorac Med 11, 269-276

Engelke C, Marten K (2007): Multislice-CT bei akuter Lungenembolie. Radiologe 47, 725-736

Engelke C, Rummeny EJ, Marten K (2006): Acute Pulmonary Embolism on MDCT of the Chest: Prediction of Cor Pulmonale and Short-Term Patient Survival from Morphologic Embolus Burden. Am J Roentgenol 186, 1265-1271 
Furlan A, Aghayev A, Chang C-CH, Patil A, Jeon KN, Park B, Fetzer DT, Saul M, Roberts MS, Bae KT (2012): Short-term Mortality in Acute Pulmonary Embolism: Clot Burden and Signs of Right Heart Dysfunction at CT Pulmonary Angiography. Radiology 265, 283-293

Geher G, Hall S (Hrsg.): Straightforward Statistics. 1. Auflage; Oxford University Press, New York 2014

Ghanima W, Abdelnoor M, Holmen LO, Nielssen BE, Sandset PM (2007): The association between the proximal extension of the clot and the severity of pulmonary embolism (PE): a proposal for a new radiological score for PE. J Intern Med 261, 74-81

Ghuysen A, Ghaye B, Willems V, Lambermont B, Gerard P, Dondelinger RF, D'Orio V (2005): Computed tomographic pulmonary angiography and prognostic significance in patients with acute pulmonary embolism. Thorax $\underline{60}$, 956-961

Goldhaber SZ: Deep Venous Thrombosis and Pulmonary Thromboembolism. In: Kasper D, Fauci A, Hauser S, Longo D, Jameson J (Hrsg.): Harrison's Principles of Internal Medicine. Band 2; 19. Auflage; McGraw-Hill Education, New York 2015, 1631-1637

Goldhaber SZ, Bounameaux H (2012): Pulmonary embolism and deep vein thrombosis. Lancet $\underline{379}$, 1835-1846

Goldhaber SZ, Visani L, Rosa MD (1999): Acute pulmonary embolism: clinical outcomes in the International Cooperative Pulmonary Embolism Registry (ICOPER). Lancet 353, 1386-1389

Gouin B, Blondon M, Jiménez D, Fernández-Capitán C, Bounameaux H, Soler S, Duce R, Sahuquillo JC, Ruiz-Giménez N, Monreal M, RIETE investigators (2017): Clinical Prognosis of Nonmassive Central and Noncentral Pulmonary Embolism: A Registry-Based Cohort Study. Chest 151, 829-837

Gussoni G, Frasson S, Regina ML, Micco PD, Monreal M (2013): Three-month mortality rate and clinical predictors in patients with venous thromboembolism and cancer. Findings from the RIETE registry. Thromb Res 131, 24-30

Hefeda MM, Elmasry MM (2014): Prediction of short term outcome of pulmonary embolism: Parameters at 16 multi-detector CT pulmonary angiography. Egypt J Radiol Nucl Med $\underline{45}$, 1089-1098

Heit JA (2008): The Epidemiology of Venous Thromboembolism in the Community. Arterioscler Thromb Vasc Biol 28, 370-372

Heit JA, Silverstein MD, Mohr DN, Petterson TM, O’Fallon WM, Melton LJ (1999): Predictors of Survival After Deep Vein Thrombosis and Pulmonary Embolism: A Population-Based, Cohort Study. Arch Intern Med 159, 445-453

Heit JA, Mohr DN, Silverstein MD, Petterson TM, O’Fallon WM, Melton LJ (2000): Predictors of recurrence after deep vein thrombosis and pulmonary embolism: a population-based cohort study. Arch Intern Med 160, 761-768

Heit JA, Spencer FA, White RH (2016): The epidemiology of venous thromboembolism. J Thromb Thrombolysis $\underline{41}, 3-14$

Henzler T, Roeger S, Meyer M, Schoepf UJ, Nance JW, Haghi D, Kaminski WE, Neumaier M, Schoenberg SO, Fink C (2012): Pulmonary embolism: CT signs and cardiac biomarkers for predicting right ventricular dysfunction. Eur Respir J 39, 919-926

Jain CC, Chang Y, Kabrhel C, Giri J, Channick R, Rodriguez-Lopez J, Rosovsky RP, Fogerty A, Rosenfield K, Jaff MR, Weinberg I (2017): Impact of Pulmonary Arterial Clot Location on Pulmonary Embolism Treatment and Outcomes (90 Days). Am J Cardiol 119, 802-807

Jardin F, Dubourg O, Bourdarias J-P (1997): Echocardiographic Pattern of Acute Cor Pulmonale. Chest $\underline{111}, 209-217$

Jiménez D, Aujesky D, Yusen RD (2010a): Risk stratification of normotensive patients with acute symptomatic pulmonary embolism. Br J Haematol $\underline{151}$, 415-424

Jiménez D, Aujesky D, Moores L, Gómez V, Lobo JL, Uresandi F, Otero R, Monreal M, Muriel A, Yusen RD (2010b): Simplification of the Pulmonary Embolism Severity Index for Prognostication in Patients With Acute Symptomatic Pulmonary Embolism. Arch Intern Med 170, 1383-1389

Jiménez D, Lobo JL, Monreal M, Moores L, Oribe M, Barrón M, Otero R, Nauffal D, Rabuñal R, Valle R, et al. (2014): Prognostic significance of multidetector CT in normotensive patients with pulmonary embolism: results of the protect study. Thorax $\underline{69}, 109-115$

Klok FA, Djurabi RK, Nijkeuter M, Eikenboom HCJ, Leebeek FWG, Kramer MHH, Kaasjager K, Kamphuisen PW, Büller HR, Huisman MV (2008): High D-dimer level is associated with increased $15-\mathrm{d}$ and 3 months mortality through a more central localization of pulmonary emboli and serious comorbidity. Br J Haematol 140, 218-222 
Klok FA, Van Der Bijl N, Eikenboom HCJ, Van Rooden CJ, De Roos A, Kroft LJM, Huisman MV (2010): Comparison of CT assessed right ventricular size and cardiac biomarkers for predicting shortterm clinical outcome in normotensive patients suspected of having acute pulmonary embolism. J Thromb Haemost $\underline{8}, 853-856$

Konstantinides S (2008): Acute Pulmonary Embolism. N Engl J Med 359, 2804-2813

Konstantinides SV, Torbicki A, Agnelli G, Danchin N, Fitzmaurice D, Galiè N, Gibbs JSR, Huisman MV, Humbert M, Kucher N, et al. (2014): 2014 ESC Guidelines on the diagnosis and management of acute pulmonary embolism. Eur Heart J $\underline{35}$, 3033-3073

Kucher N, Rossi E, De Rosa M, Goldhaber SZ (2005): Prognostic role of echocardiography among patients with acute pulmonary embolism and a systolic arterial pressure of $90 \mathrm{~mm} \mathrm{Hg}$ or higher. Arch Intern Med 165, 1777-1781

Lankeit M (Hrsg.): Diagnostik und Therapie der akuten Lungenembolie. 2. Auflage; UNI-MED, Bremen 2016

Lankeit M (2017): Always think of the right ventricle, even in „low-risk“ pulmonary embolism. Eur Respir J 50, 1702386

Lankeit M, Friesen D, Aschoff J, Dellas C, Hasenfuß G, Katus H, Konstantinides S, Giannitsis E (2010): Highly sensitive troponin $\mathrm{T}$ assay in normotensive patients with acute pulmonary embolism. Eur Heart J $\underline{31}, 1836-1844$

Lankeit M, Jiménez D, Kostrubiec M, Dellas C, Hasenfuss G, Pruszczyk P, Konstantinides S (2011): Predictive Value of the High-Sensitivity Troponin T Assay and the Simplified Pulmonary Embolism Severity Index in Hemodynamically Stable Patients With Acute Pulmonary Embolism Clinical Perspective. Circulation 124, 2716-2724

Lankeit M, Jiménez D, Kostrubiec M, Dellas C, Kuhnert K, Hasenfuß G, Pruszczyk P, Konstantinides S (2014): Validation of N-terminal pro-brain natriuretic peptide cut-off values for risk stratification of pulmonary embolism. Eur Respir J 43, 1669-1677

Lankhaar J-W, Westerhof N, Faes TJC, Marques KMJ, Marcus JT, Postmus PE, Vonk-Noordegraaf A (2006): Quantification of right ventricular afterload in patients with and without pulmonary hypertension. Am J Physiol Heart Circ Physiol 291, H1731-H1737

Lu MT, Demehri S, Cai T, Parast L, Hunsaker AR, Goldhaber SZ, Rybicki FJ (2012): Axial and Reformatted Four-Chamber Right Ventricle-to-Left Ventricle Diameter Ratios on Pulmonary CT Angiography as Predictors of Death After Acute Pulmonary Embolism. Am J Roentgenol 198, 1353-1360

Mastora I, Remy-Jardin M, Masson P, Galland E, Delannoy V, Bauchart J-J, Remy J (2003): Severity of acute pulmonary embolism: evaluation of a new spiral CT angiographic score in correlation with echocardiographic data. Eur Radiol 13, 29-35

McIntyre KM, Sasahara AA (1971): The hemodynamic response to pulmonary embolism in patients without prior cardiopulmonary disease. Am J Cardiol 28, 288-294

van der Meer RW, Pattynama PMT, van Strijen MJL, van den Berg-Huijsmans AA, Hartmann IJC, Putter H, de Roos A, Huisman MV (2005): Right Ventricular Dysfunction and Pulmonary Obstruction Index at Helical CT: Prediction of Clinical Outcome during 3-month Follow-up in Patients with Acute Pulmonary Embolism. Radiology 235, 798-803

Meinel FG, Nance Jr. JW, Schoepf UJ, Hoffmann VS, Thierfelder KM, Costello P, Goldhaber SZ, Bamberg F (2015): Predictive Value of Computed Tomography in Acute Pulmonary Embolism: Systematic Review and Meta-analysis. Am J Med 128, 747-759.e2

Olié V, Fuhrman C, Chin F, Lamarche-Vadel A, Scarabin P-Y, de Peretti C (2015): Time trends in pulmonary embolism mortality in France, 2000-2010. Thromb Res 135, 334-338

Ozsu S, Karaman K, Mentese A, Ozsu A, Karahan SC, Durmus I, Oztuna F, Kosucu P, Bulbul Y, Ozlu T (2010): Combined risk stratification with computerized tomography / echocardiography and biomarkers in patients with normotensive pulmonary embolism. Thromb Res $\underline{126}$, 486-492

Patel S, Kazerooni EA, Cascade PN (2003): Pulmonary Embolism: Optimization of Small Pulmonary Artery Visualization at Multi-Detector Row CT. Radiology 227, 455-460

Pollack CV, Schreiber D, Goldhaber SZ, Slattery D, Fanikos J, O’Neil BJ, Thompson JR, Hiestand B, Briese BA, Pendleton RC, et al. (2011): Clinical Characteristics, Management, and Outcomes of Patients Diagnosed With Acute Pulmonary Embolism in the Emergency Department: Initial Report of EMPEROR (Multicenter Emergency Medicine Pulmonary Embolism in the Real World Registry). J Am Coll Cardiol 57, 700-706 
Prandoni P, Lensing AW, Cogo A, Cuppini S, Villalta S, Carta M, Cattelan AM, Polistena P, Bernardi E, Prins MH (1996): The long-term clinical course of acute deep venous thrombosis. Ann Intern Med $\underline{125}, 1-7$

Prins MH, Lensing AWA, Prandoni P, Wells PS, Verhamme P, Beyer-Westendorf J, Bauersachs R, Bounameaux H, Brighton TA, Cohen AT, et al. (2018): Risk of recurrent venous thromboembolism according to baseline risk factor profiles. Blood Adv 2, 788-796

Pruszczyk P, Pacho R, Ciurzynski M, Kurzyna M, Burakowska B, Tomkowski W, Bochowicz A, Torbicki A (2003): Short term clinical outcome of acute saddle pulmonary embolism. Heart $\underline{89}$, 335-336

Puls M, Dellas C, Lankeit M, Olschewski M, Binder L, Geibel A, Reiner C, Schäfer K, Hasenfuss G, Konstantinides S (2007): Heart-type fatty acid-binding protein permits early risk stratification of pulmonary embolism. Eur Heart J 28, 224-229

Qanadli SD, El Hajjam M, Vieillard-Baron A, Joseph T, Mesurolle B, Oliva VL, Barré O, Bruckert F, Dubourg O, Lacombe P (2001): New CT Index to Quantify Arterial Obstruction in Pulmonary Embolism. Am J Roentgenol 176, 1415-1420

Reid JH, Murchison JT (1998): Acute right ventricular dilatation: a new helical CT sign of massive pulmonary embolism. Clin Radiol $\underline{53}$, 694-698

Rodrigues B, Correia H, Figueiredo A, Delgado A, Moreira D, Ferreira Dos Santos L, Correia E, Pipa J, Beirão I, Santos O (2012): [Clot burden score in the evaluation of right ventricular dysfunction in acute pulmonary embolism: quantifying the cause and clarifying the consequences]. Rev Port Cardiol 31, 687-695

Sanchez O, Trinquart L, Caille V, Couturaud F, Pacouret G, Meneveau N, Verschuren F, Roy P-M, Parent F, Righini M, et al. (2010): Prognostic Factors for Pulmonary Embolism. Am J Respir Crit Care Med 181, 168-173

Schoepf UJ, Holzknecht N, Helmberger TK, Crispin A, Hong C, Becker CR, Reiser MF (2002): Subsegmental pulmonary emboli: improved detection with thin-collimation multi-detector row spiral CT. Radiology 222, 483-490

Schoepf UJ, Kucher N, Kipfmueller F, Quiroz R, Costello P, Goldhaber SZ (2004): Right Ventricular Enlargement on Chest Computed Tomography. Circulation 110, 3276-3280

Senturk A, Ozsu S, Duru S, Cakır E, Ulaslı SS, Demirdogen E, Kayhan S, Guzel A, Yakar F, Berk S (2017): Prognostic importance of central thrombus in hemodynamically stable patients with pulmonary embolism. Cardiol J 24, 508-514

Stein PD, Fowler SE, Goodman LR, Gottschalk A, Hales CA, Hull RD, Leeper KVJ, Popovich JJ, Quinn DA, Sos TA, et al. (2006): Multidetector Computed Tomography for Acute Pulmonary Embolism. N Engl J Med 354, 2317-2327

Subramaniam RM, Mandrekar J, Chang C, Blair D, Gilbert K, Peller PJ, Sleigh J, Karalus N (2008): Pulmonary Embolism Outcome: A Prospective Evaluation of CT Pulmonary Angiographic Clot Burden Score and ECG Score. Am J Roentgenol 190, 1599-1604

Tamizifar B, Fereyduni F, Esfahani MA, Kheyri S (2016): Comparing three clinical prediction rules for primarily predicting the 30-day mortality of patients with pulmonary embolism: The "Simplified Revised Geneva Score," the "Original PESI," and the "Simplified PESI". Adv Biomed Res $\underline{5,137}$

Tapson VF (2008): Acute Pulmonary Embolism. N Engl J Med 358, 1037-1052

ten Wolde M, Söhne M, Quak E, Mac Gillavry MR, Büller HR (2004): Prognostic value of echocardiographically assessed right ventricular dysfunction in patients with pulmonary embolism. Arch Intern Med $\underline{164}, 1685-1689$

Torbicki A, Perrier A, Konstantinides S, Agnelli G, Galiè N, Pruszczyk P, Bengel F, Brady AJB, Ferreira D, Janssens U, et al. (2008): Guidelines on the diagnosis and management of acute pulmonary embolism. Eur Heart J 29, 2276-2315

Trujillo-Santos J, den Exter PL, Gómez V, del Castillo H, Moreno C, van der Hulle T, Huisman MV, Monreal M, Yusen RD, Jiménez D (2013): Computed tomography-assessed right ventricular dysfunction and risk stratification of patients with acute non-massive pulmonary embolism: systematic review and meta-analysis. J Thromb Haemost $\underline{11}$, 1823-1832

Vedovati MC, Becattini C, Agnelli G, Kamphuisen PW, Masotti L, Pruszczyk P, Casazza F, Salvi A, Grifoni S, Carugati A, et al. (2012): Multidetector CT scan for acute pulmonary embolism: embolic burden and clinical outcome. Chest 142, 1417-1424 
Vedovati MC, Germini F, Agnelli G, Becattini C (2013): Prognostic role of embolic burden assessed at computed tomography angiography in patients with acute pulmonary embolism: systematic review and meta-analysis. J Thromb Haemost 11, 2092-2102

Wells PS, Anderson DR, Rodger M, Ginsberg JS, Kearon C, Gent M, Turpie AGG, Bormanis J, Weitz J, Chamberlain M, et al. (2000): Derivation of a Simple Clinical Model to Categorize Patients Probability of Pulmonary Embolism: Increasing the Models Utility with the SimpliRED D-dimer. Thromb Haemost $\underline{83}, 416-420$

Wells PS, Anderson DR, Rodger M, Stiell I, Dreyer JF, Barnes D, Forgie M, Kovacs G, Ward J, Kovacs MJ (2001): Excluding pulmonary embolism at the bedside without diagnostic imaging: management of patients with suspected pulmonary embolism presenting to the emergency department by using a simple clinical model and d-dimer. Ann Intern Med 135, 98-107

White RH (2003): The Epidemiology of Venous Thromboembolism. Circulation 107, I-4-I-8

Wintersperger BJ, Stäbler A, Seemann M, Holzknecht N, Helmberger T, Fink U, Reiser MF (1999): Beurteilung der Rechtsherzbelastung in der Spiral-CT bei Patienten mit akuter Lungenembolie. Fortschr Röntgenstr 170, 542-549

Wu AS, Pezzullo JA, Cronan JJ, Hou DD, Mayo-Smith WW (2004): CT pulmonary angiography: quantification of pulmonary embolus as a predictor of patient outcome--initial experience. Radiology 230, 831-835 


\section{Danksagung}

Mein besonderer Dank gilt Frau Prof. Dr. med. C. Dellas für die freundliche Überlassung des Themas und ihre enge und engagierte Betreuung und Beratung bei der Ausarbeitung. Ihre stetige Unterstützung bei Fragen und Problemen aller Art und ihre konstruktiven Anregungen haben maßgeblich zur Fertigstellung dieser Arbeit beigetragen.

Ganz herzlich möchte ich mich auch bei meinem Betreuer Dr. med. K. Hellenkamp bedanken, der mir jederzeit mit Rat und Tat zur Seite stand. Seine wertvollen Anregungen, seine konstruktive Kritik und der herzliche Umgang haben einen wichtigen Beitrag zur Erstellung dieser Arbeit geleistet.

Großer Dank gilt auch Frau PD Dr. med. M. Lankeit für die freundliche Zusammenarbeit innerhalb der Arbeitsgruppe und Herrn PD Dr. J. M. Sommerlath Sohns, der mir mit seiner Fachkenntnis zur Seite stand.

Weiterhin gilt mein Dank allen Mitarbeiter/innen der Abteilung für Diagnostische Radiologie, der Internistischen Notaufnahme und der Abteilung für Klinische Chemie der Universitätsmedizin Göttingen, die mich stets unterstützend und freundlich bei meiner Arbeit begleitet haben. Besonderer Dank gilt hier Herrn Bodo Schulz für seine unermüdliche Hilfsbereitschaft. 